\title{
Micro/Nano Amorphization/Oxidation of Silicon Induced by High Repetition Femtosecond Laser Pulses
}

\author{
by \\ Amirkianoosh Kiani, B.Sc, M.Sc., \\ Isfahan University of Technology, Isfahan, Iran, 2003, \\ National Technical University of Ukraine, Kiev, Ukraine, 2007, \\ A dissertation presented to Ryerson University \\ in partial fulfilment of the requirement for the degree of \\ Doctor of Philosophy in the Program of \\ Mechanical and Industrial Engineering.
}

Toronto, Ontario, Canada, 2013

(C) Amirkianoosh Kiani 2013 


\section{Author's Declaration}

I hereby declare that I am the sole author of this dissertation. This is a true copy of the dissertation, including any required final revisions, as accepted by my examiners.

I authorize Ryerson University to lend this dissertation to other institutions or individuals for the purpose of scholarly research

Amirkianoosh Kiani

I further authorize Ryerson University to reproduce this dissertation by photocopying or by other means, in total or in part, at the request of other institutions or individuals for the purpose of scholarly research.

I understand that my dissertation may be made electronically available to the public. Amirkianoosh Kiani 


\begin{abstract}

\section{Micro/Nano Amorphization/Oxidation of Silicon Induced by High Repetition Femtosecond Laser Pulses}

\author{
(C) Amirkianoosh Kiani 2013 \\ Doctor of Philosophy \\ in the Program of \\ Mechanical and Industrial Engineering, \\ Ryerson University.
}

The main aim of this thesis is to develop a new method for direct micro/nano amorphization/oxidation of silicon using femtosecond laser irradiation and its applications in maskless lithography and solar cell fabrication.

Amorphization and oxidation occur when crystalline silicon is exposed to the irradiation of femtosecond laser pulses below the ablation threshold. Mechanisms of amorphization and oxidation were discussed and the surface temperature model was developed to study the relation between laser parameters and observed amorphization and oxidation. A systematic theoretical and experimental study of the influence of the laser parameters on the quality of amorphorized area and the size of the feature fabricated through amorphization has been studied. It was found that during the process of silicon amorphization and oxidation, the 
higher repetition rate of laser pulses yields smooth morphology with better repeatability. Increasing pulse duration and number of pulses were seen to increase the line width. However, increasing the number of pulses does not result in ablation of the target area. An analytical model was developed for the calculation of the average surface temperature after n-pulses.

The effect of the laser pulse width was investigated by developing an analytical model for the calculation of the non-dimensional surface temperature with various pulse widths. It was found from experimental and analytical results that for a constant power and repetition rate, an increase in the pulse duration corresponds to a significant increase in the surface temperature. It results in an increase in the amount of modified material as well as improvement of light absorption in the case of amorphization.

The amorphous silicon and silicon oxide can act as an etch stop. Therefore, maskless lithography is possible with the direct patterning (amorphization and oxidation) of crystalline silicon. Experimental results have proved the feasibility of the proposed concepts. The thin-film of amorphous silicon generated on the silicon substrate has a potential for use in photovoltaic devices and solar cell fabrication. In comparison with previous methods, the direct oxidation/amorphization of silicon induced by the femtosecond laser is a maskless single-step technique which offers a higher flexibility and reduced processing time. 


\section{Acknowledgements}

I would like to acknowledge my supervisor Dr. Krishnan Venkatakrishnan and Dr. Bo Tan who have been a strong source of inspiration throughout my project work. I have benefited greatly from their invaluable guidance and motivation. Their guidance and moral support has helped me to succeed beyond my wildest dreams.

I would also like to thank my parents, Rezvan and Bakhtiar, for their continual support and encouragement, which has given me the ability to pursue my goals with confidence and full determination. I am forever grateful to them for this. I would also like to express

my special thanks to my wife, Mehrnaz Shokrollahi and my brother Amirkiarash Kiani. Their motivation, support and encouragement gave me the strength to peruse my goals and dreams. This thesis would never have been completed without their help and support specially in preparing the IATEX version of my thesis. I am forever delighted and thankful for having them in my life. I also would like to thank my sister Anahita Kiani and my brother-in-law Adrian Jakibchuk. Their support, encouragement and love motivated me to pursue my PhD at Ryerson University.

I would also like to express my profound gratitude to the committee members, Dr. Hua Lu and Dr. Ahmad Ghasempoor for their constructive comments, suggestions and guidance. 
I also would like to thank my fellow colleagues from the "Laser micro/nano fabrication laboratory" (Ryerson University), especially Amirhossein Tavangar and Priyatha Premnath for their enlightening and informative discussions they had with me about my research.

Last but not least, I would like to thank everyone at Ryerson University who provided me with an enhanced educational experience, in which I will never forget. 


\section{Dedication}

I lovingly dedicate this thesis to the love of my life Mehrnaz and my only brother

Amirkiarash as a token of humble and sincere appreciation for their invaluable love, enormous support and continuous encouragement... 


\section{Table of Contents}

List of Figures $\quad$ xii

List of Tables $\quad$ xvii

List of Acronyms $\quad$ xviii

List of Symbols $\quad$ Xx

1 Introduction $\quad 1$

1.1 Thin-film Silicon (amorphorized/oxidized) . . . . . . . . . . . . 1

1.2 Fabrication Methods of Amorphorized/Oxidized Thin-Film . . . . . . . . 2

1.2.1 Chemical Vapor Deposition (CVD): large area oxidization/

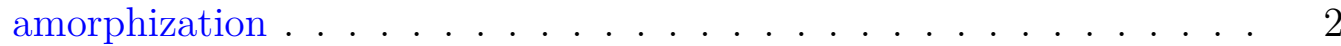

1.2.2 Patterning of amorphorized-oxidized thin-film silicon in micro/nano scale .......................... . . . 4 
1.3 Laser Processing of Silicon . . . . . . . . . . . . . . . . . . . . . . . 8

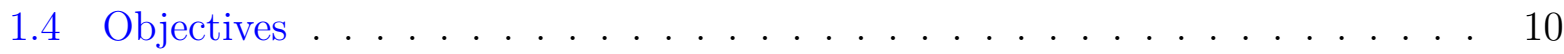

2 Physics of Laser Annealing of Silicon $\quad 11$

2.1 Properties of Silicon _. . . . . . . . . . . . . . . . . . . . . 11

2.2 Laser Annealing of Silicon . . . . . . . . . . . . . . . . . . . . . . 13

2.2.1 Irradiation of a solid target by a single laser pulse . . . . . . . . . 13

2.2.2 Surface temperature of the target in the case of a succession of laser

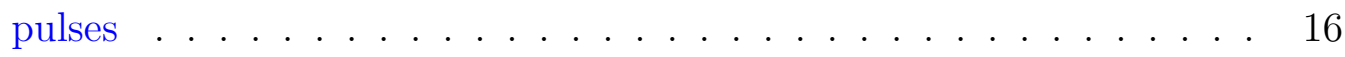

2.2.3 Non-dimensional surface temperature of laser irradiation and effect of laser parameters . . . . . . . . . . . . . . . . 20

3 Experimental Setup and Procedure $\quad 26$

3.1 Laser System . . . . . . . . . . . . . . . . . . . . . . 26

3.1 .1 Optical Setup . . . . . . . . . . . . . . . . . 27

3.2 Samples, Procedure and Analyzing _ . . . . . . . . . . . . . . . . . . 29

4 Direct-writing of Oxidized Silicon Thin-film on Si Substrate 31

4.1 Experimental Results . . . . . . . . . . . . . . . . . . . . . . . . 32

4.2 Numerical Model for Surface Temperature Calculation . . . . . . . . . . . . 34

4.3 Summary . . . . . . . . . . . . . . . . . . . . . . . . . 39 
5 Direct-writing of Amorphorized Silicon Thin-film on Si Substrate

5.1 Procedure . . . . . . . . . . . . . . . . . . . . . . . 41

5.2 Results and Discussion . . . . . . . . . . . . . . . . . . . . . . . 42

5.3 Summary . . . . . . . . . . . . . . . . . . . . . . 49

$6 \quad$ Effect of Laser Parameters $\quad 51$

6.1 Laser Parameters and Laser Annealing of Silicon . . . . . . . . . . . . . . . 51

6.2 Experimental Setup . . . . . . . . . . . . . . . . . . . 53

6.3 Results and Discussion . . . . . . . . . . . . . . . . . . . . . 53

6.3 .1 Effect of pulse repetition . . . . . . . . . . . . . . 54

6.3 .2 Effect of pulse duration . . . . . . . . . . . . . . 55

6.3 .3 Effect of number of laser pulses . . . . . . . . . . . . . 60

6.4 Summary . . . . . . . . . . . . . . . . . . . . . . . . . 69

7 Application of Amorphorized/Oxidized Silicon Thin-film $\quad 71$

7.1 Maskless Lithography Using Oxidized/Amorphorized Silicon Etch-stop Layer Induced by Mega Hertz Repetition Femtosecond Laser Pulses . . . . . . . . . 72

7.1.1 Maskless lithography using thin-film silicon oxide as an etch stop . . 75

7.1.2 Maskless lithography using amorphous silicon etch-stop layer . . . . . 80 
7.2 Enhancement of the Optical Absorption of Thin-film of Amorphorized Silicon for Photovoltaic Energy Conversion . . . . . . . . . . . . . . . . . . 85

7.2 .1 Results and Discussion . . . . . . . . . . . . . . . . . . . . 88

7.3 Summary . . . . . . . . . . . . . . . . . . . . . . . 95

8 Summary and Future Research $\quad 97$

8.1 Summary . . . . . . . . . . . . . . . . . . . . . . . . . 97

8.2 Further Research . . . . . . . . . . . . . . . . . . . . . . . . . . 101

\section{Appendices}

A Properties of Silicon at Room Temperature

$\begin{array}{ll}\text { B List of Publications } & 107\end{array}$

1 Refereed Journals . . . . . . . . . . . . . . . . . . . . . . 107

2 Refereed Conference Papers . . . . . . . . . . . . . . . . . . 108

3 Submitted to Refereed Journals . . . . . . . . . . . . . . . . . . . 109

$\begin{array}{ll}\text { References } & 110\end{array}$ 


\section{List of Figures}

1.1 Process of scanning probe oxidation of silicon $\ldots \ldots \ldots 6$

1.2 Process of Tribo-nanolithography of $\mathrm{Si} \ldots \ldots \ldots$

2.1 Different Si forms (crystalline, multicrystalline and amorphous) . . . . . 12

2.2 Temperature of the target surface heated by a series of laser pulses $\ldots \ldots 17$

3.1 Experimental setup for amorphization process . . . . . . . . . . . 28

3.2 Experimental setup for oxidation process $\ldots \ldots \ldots 30$

4.1 Scanning Electron Microscope (SEM) image of silicon surface after irradiation with Femtosecond Laser Pulses at $1 \mathrm{~W}$ at the various pulse frequency (left to

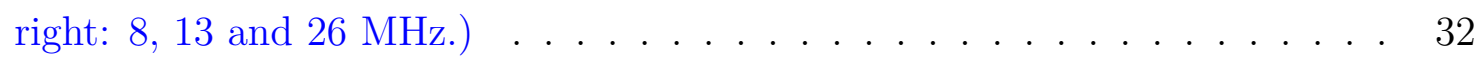

4.2 SEM image of silicon surface after irradiation with Femtosecond Laser pulses at $0.35 \mathrm{~W}$ at the pulse Frequency of $26 \mathrm{MHz} \ldots \ldots \ldots . \ldots . \ldots 33$ 
4.3 (a) Optical microscope topography and cross sectional image $(26 \mathrm{MHz}, 0.35$ W), (b) optical microscope topography and cross sectional image $(13 \mathrm{MHz}$,

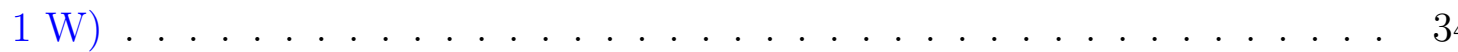

4.4 (a) Graph of Energy Dispersive X-ray (EDX) spectroscopy analysis (b) Graph of Micro-Raman spectroscopy analysis . . . . . . . . . . . . . . . . 35

4.5 (a) theoretical results based on Equation 4.4 (Relation between $\mathrm{T}$ and frequency at various powers) and (b)Experimental results . . . . . . . . .

5.1 Process of experiment . . . . . . . . . . . . . . . . . . . 41

5.2 (a) Schematic drawing of laser intensity and surface modification on silicon substrate. (b) Silicon wafer after irradiation with sub-threshold. (c) Silicon wafer after etching $\ldots \ldots \ldots \ldots \ldots \ldots \ldots \ldots \ldots$

5.3 SEM image of ring feature after etching in $\mathrm{KOH}$ solution for 15 min at $45{ }^{\circ} \mathrm{C}$ (Average laser fluence: $\left.0.27 \mathrm{~J} / \mathrm{cm}^{2}\right) \ldots \ldots \ldots \ldots$. . . . . . . . 44

5.4 SEM images features after 3 min etching (a) ring feature at average laser fluence of $0.3 \mathrm{~J} / \mathrm{cm}^{2}$ (b) solid round at $0.2 \mathrm{~J} / \mathrm{cm}^{2} \ldots \ldots \ldots \ldots$

5.5 SEM image (at the incident angle of $35^{\circ}$ ) of line feature after etching in $\mathrm{KOH}$ solution (Pulse energy: $\left.0.28 \mathrm{~J} / \mathrm{cm}^{2}\right) \ldots \ldots \ldots \ldots \ldots$

5.6 (a) $5 \mu \mathrm{m}$ linewidth at pulse energy of $0.28 \mathrm{~J} / \mathrm{cm}^{2}$ (at $26 \mathrm{MHz}$ ) (a) $2.5 \mu \mathrm{m}$ linewidth at pulse energy of $0.24 \mathrm{~J} / \mathrm{cm}^{2}$ (at $26 \mathrm{MHz}$ ) (b) $1.0 \mu \mathrm{m}$ linewidth at pulse energy of $0.2 \mathrm{~J} / \mathrm{cm}^{2}$ (at $26 \mathrm{MHz}$ ) (c) $150 \mathrm{~nm}$ linewidth at pulse energy of $0.08 \mathrm{~J} / \mathrm{cm}^{2}($ at $26 \mathrm{MHz}) \ldots \ldots \ldots \ldots \ldots \ldots$ 
5.7 EDX analysis of (a) irradiated zone and (b) non-irradiated zone . . . . . . . 48

5.8 Raman spectroscopy graph . . . . . . . . . . . . . . . . . . . . . . 49

6.1 SEM images at different pulse repetition rates (pulse duration: $214 \mathrm{fs}$ ), (a) 8 MHz, $4.8 \mathrm{~W}$, (b) $13 \mathrm{MHz}, 5.6 \mathrm{~W}$, and (c) $26 \mathrm{MHz}, 6.25 \mathrm{~W} \ldots \ldots . . .54$

6.2 SEM images at different pulse width at the scanning speed of $75 \mathrm{~mm} / \mathrm{s}$, pulse repetition of $26 \mathrm{MHz}$ and the power of $6 \mathrm{~W}$, (a) $214 \mathrm{fs} 4.7 \mu \mathrm{m}$, (b) $720 \mathrm{fs}$, $5.3 \mu \mathrm{m}$, and (c) $3500 \mathrm{fs}, 7.2 \mu \mathrm{m} \ldots \ldots \ldots \ldots \ldots \ldots$

6.3 Width of amorphorized zone vs pulse width for $214 \mathrm{fs}, 720 \mathrm{fs}$, and $3500 \mathrm{fs}$ (26 $\mathrm{MHz}) \ldots \ldots \ldots \ldots \ldots \ldots \ldots \ldots \ldots \ldots \ldots \ldots$

6.4 Computed non-dimensional surface temperature constant amount of power (a) and energy $(\mathrm{b}) \ldots \ldots \ldots \ldots \ldots$

6.5 Analytical/experimental graphs of: a) non-dimensional surface temperature, b) raman peak of amorphous silicon, c) light absorption at $630 \mathrm{~nm} \quad \ldots . .60$

6.6 SEM images of silicon samples after irradiated with 3500 fs laser at different number of pulses (26 MHz), (a) $25 \mathrm{~mm} / \mathrm{s}$, (b) $50 \mathrm{~mm} / \mathrm{s}$, (c) $75 \mathrm{~mm} / \mathrm{s}$, and

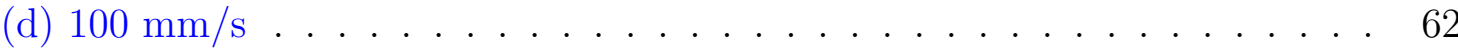

6.7 Width of amorphorized zone vs number of pulses for 2000 to $11000(26 \mathrm{MHz}) 63$

6.8 Change in surface temperature at different pulse repetitions and powers (pulse width of $214 \mathrm{fs}) \ldots \ldots \ldots \ldots \ldots \ldots \ldots$

$6.9 \phi(N)$ vs number of pulses $(26 \mathrm{MHz}) \ldots \ldots \ldots \ldots \ldots$ 
7.1 a) conventional lithography method, b) direct (single-step) silicon oxide patterning, c) direct maskless lithography induced by fs laser . . . . . . . . . . 73

7.2 SEM images of silicon oxide pattern induced by $214 \mathrm{fs}$ laser pulses at the frequency of $26 \mathrm{MHz}$ and the average power of $3.3 \mathrm{~W}\left(I=0.15 \mathrm{~J} / \mathrm{cm}^{2}\right) \quad \ldots \quad 75$

7.3 Optical microscope topography and cross sectional images (a) after irradiation (height: 500nm) and (b) after etching process (height: $1000 \mathrm{~nm}$ ) . . . . 76

7.4 EDX results of irradiated area . . . . . . . . . . . . . . 77

7.5 micro-Raman spectroscopy results of irradiated samples before/after etching

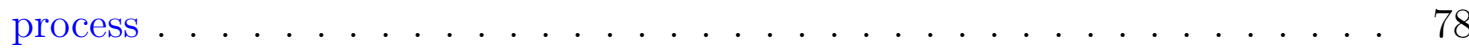

7.6 X-Ray Diffraction (XRD) results of pre/post-irradiated samples. XRD spectrum of pre-irradiated area is dominated by the signals at $2 \Theta=28,56$ and 47 (zoomed chart) which corresponds to crystalline silicon. The XRD measurements on the processed samples shows only a peak shifted to lower level

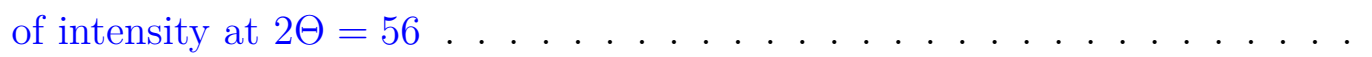

7.7 SEM images of silicon oxide pattern after etching in $\mathrm{KOH}(30 \%)$ at $65^{\circ} \mathrm{C}$ after

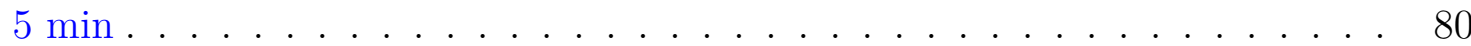

7.8 Process of experiment $\ldots \ldots \ldots \ldots \ldots$

7.9 SEM images of irradiated samples during etching process . . . . . . . . . . 82

7.10 SEM image of feature after 3 min etching - solid round at $0.2 \mathrm{~J} / \mathrm{cm}^{2} \ldots \ldots 83$

7.11 SEM image at the incident angle of $35^{\circ}$ of line feature after etching in $\mathrm{KOH}$ (Pulse energy: $\left.0.28 \mathrm{~J} / \mathrm{cm}^{2}\right) \ldots \ldots \ldots \ldots \ldots \ldots$ 
7.12 SEM images at different pulse repetition rates (pulse duration: $214 \mathrm{fs}$ ) . . . . 84

$7.13150 \mathrm{~nm}$ line width at pulse energy of $0.1 \mathrm{~J} / \mathrm{cm}^{2}($ at $26 \mathrm{MHz}) \ldots \ldots$

7.14 EDX analysis of irradiated zone . . . . . . . . . . . . 86

7.15 Raman spectroscopy graph . . . . . . . . . . . . . . . . . 87

7.16 SEM images of amorphorized silicon (Frequency: $26 \mathrm{MHz}$, Power: $5.4 \mathrm{~W}$, Scanning speed: $50 \mathrm{~mm} / \mathrm{s}$, Pulse duration: $3500 \mathrm{fs}$ (left), $1400 \mathrm{fs}$ (right)) . 89

7.17 EDX results of irradiated area (samples were coated with gold for better spectroscopy results $) \ldots \ldots \ldots \ldots$

7.18 Mico-Raman spectroscopy results of amorphorized silicon with various pulse durartion $(200$ to $3500 \mathrm{fs}) \ldots \ldots \ldots \ldots \ldots$

7.19 Scanning near-field optical microscopy of irradiated samples (Left: surface morphology, Right: light absorption analysis) _. . . . . . . . . . . . 91

7.20 Light spectroscopy of amorphorized area induced by laser pulses with (a) different pulse duration and (b) scanning speed $\ldots \ldots \ldots$. . . . . . . 92

7.21 Analytical/experimental graphs of: a) non-dimensional surface temperature, b) raman peak of amorphous silicon, c) light absorption at $630 \mathrm{~nm} \ldots \ldots . .94$ 


\section{List of Tables}

1.1 Type of Chemical Vapor Deposition (CVD) methods and their principal char-

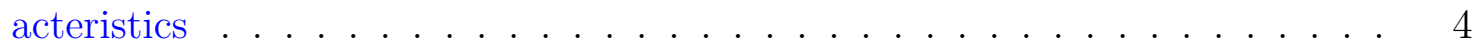

6.1 Laser parameters for the oxidation and amorphization process . . . . . . . 52

6.2 Laser fluence at different powers and repetition rates at the pulse duration of

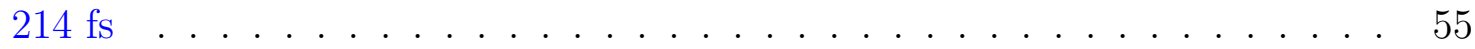

6.3 Analytical and experimental results of the amorphorized silicon wafers . . . 60

$6.4 \phi_{t h}(\mathrm{~N})$ at different powers and repetition rates (pulse duration: $214 \mathrm{fs}$ ). . . 69

7.1 Analytical and experimental results of the amorphorized silicon wafers . . . . 93

A.1 Properties of silicon at room temperature . . . . . . . . . . . . 106 


\section{List of Acronyms}

AFM Atomic Force Microscope

APCVD Atmospheric Pressure $C V D$

CAD Computer-Aided Design

CVD Chemical Vapor Deposition

DPL Dip-Pen Lithography

DPN Dip-Pen Nanolithography

EB Electron Beam

EDX Energy Dispersive X-ray

ESINL Easy Soft Imprint Nanolithography

FIB Focused Ion Beam

HDPCVD High Density Plasma CVD

HAZ Heated Affected Zone

IC Integrated Circuit

IL Interference Lithography 
LOC Lab-On-a-Chip

LPCVD Low Pressure $C V D$

LPE Liquid Phase Epitaxy

MAEBL multi-axis electron beam lithography

MEMS Micro Electro-Mechanical Systems

MOPL Maskless Optical-Projection Lithograph

NA Numerical Aperture

NEMS Nano Electro Mechanical System

PECVD Plasma Enhanced $C V D$

SEBL Scanning Electron Beam Lithography

SEM Scanning Electron Microscope

SNOM Scanning Near-field Optical Microscopy

SPL Scanning-Probe Lithography

STM Scanning Transmission Microscopy

TEM Transmission Electron Microscopy

TEOS TetraEthyl OrthosSilicate

TF-Si Thin-Film Silicon

TNL Tribo Nano Lithography

XRD X-Ray Diffraction 


\section{List of Symbols}

\section{Mathematics}

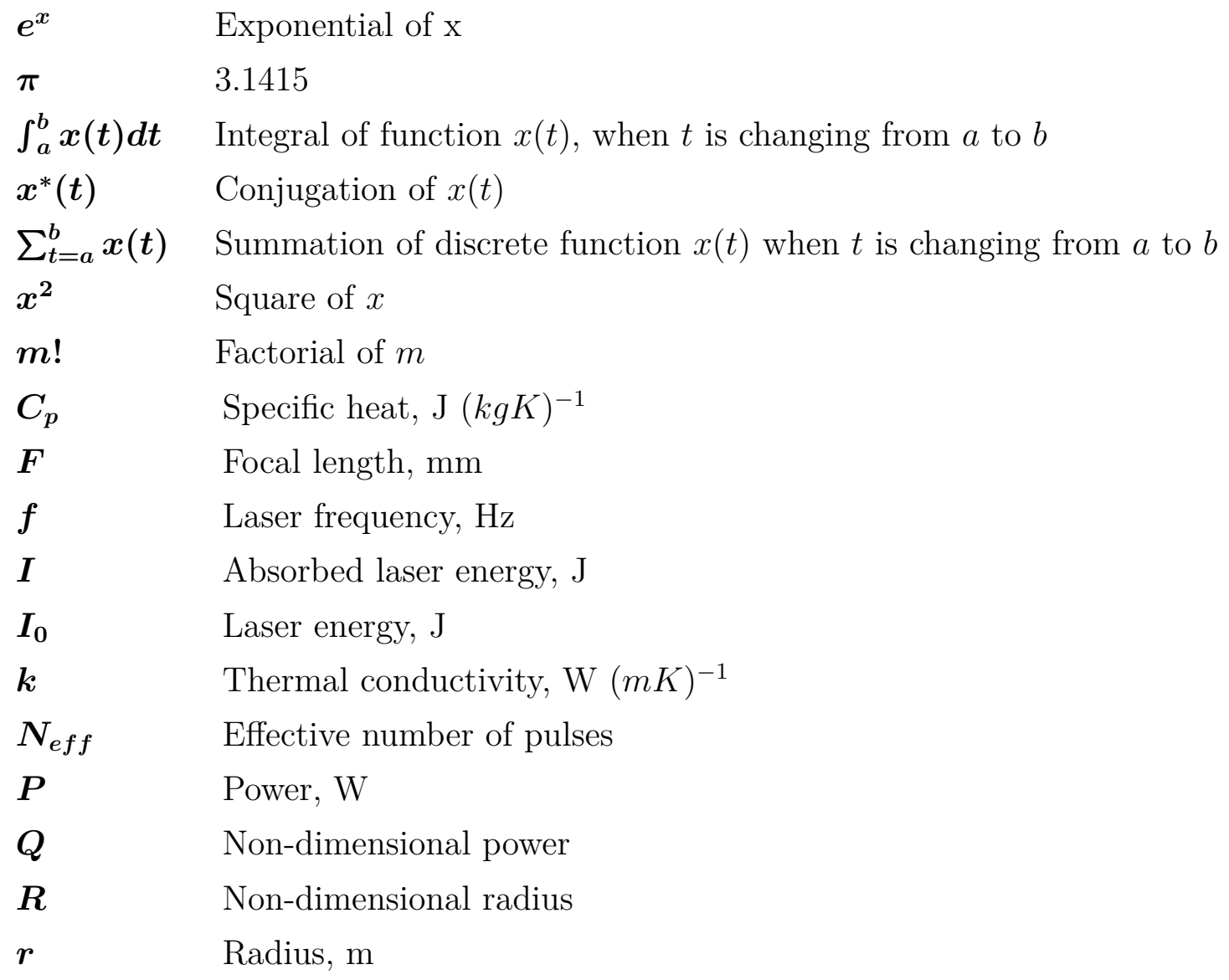




$\begin{array}{ll}\boldsymbol{r}_{\mathbf{0}} & \text { Diameter of the beam spot, } \mathrm{m} \\ \boldsymbol{T} & \text { Temperature, K } \\ \boldsymbol{T}_{\boldsymbol{r} \boldsymbol{f}} & \text { Reference temperature, K } \\ \boldsymbol{t} & \text { Time, s } \\ \boldsymbol{t}_{\boldsymbol{p}} & \text { Pulse width (duration), s } \\ \boldsymbol{U}(\boldsymbol{t}) & \text { Unit step function } \\ \boldsymbol{V} & \text { Scanning speed, } \mathrm{m} / \mathrm{s} \\ \boldsymbol{v} & \text { Volume, } \mathrm{m}^{3} \\ \boldsymbol{Z} & \text { Non-dimensional height } \\ \boldsymbol{z} & \text { Height, } \mathrm{m}\end{array}$

Greeks

$\begin{array}{ll}\boldsymbol{\alpha} & \text { Thermal diffusion } \\ \boldsymbol{\eta} & \text { Fraction of the surface absorption } \\ \boldsymbol{\theta} & \text { Non-dimensional temperature } \\ \boldsymbol{\lambda} & \text { Space dimension } \\ \boldsymbol{\mu} & \text { Spot diameter } \\ \boldsymbol{\nu} & \text { Scanning Speed } \\ \boldsymbol{\omega}_{\mathbf{0}} & \text { Machining spot radius, } \mathrm{m} \\ \boldsymbol{\rho} & \text { Density } \mathrm{kg} / \mathrm{m}^{3} \\ \boldsymbol{\tau} & \text { Non-dimensional time } \\ \boldsymbol{\tau}_{\boldsymbol{p}} & \text { Non-dimensional pulse width } \\ \boldsymbol{\phi} & \text { Amorphization Threshold }\end{array}$




\section{Chemical Elements}

\begin{tabular}{ll}
\hline & \\
$\mathbf{H}$ & Hydrogen \\
$\mathbf{C}$ & Carbon \\
$\mathbf{N}$ & Nitrogen \\
$\mathbf{O}$ & Oxygen \\
$\mathrm{Si}$ & Silicon \\
$\mathbf{K}$ & Potassium
\end{tabular}




\section{Chapter 1}

\section{Introduction}

\subsection{Thin-film Silicon (amorphorized/oxidized)}

\footnotetext{
S ILICON thin-film in various forms (such as amorphorized, oxidized, nitride, crystalline and etc.) is used not only for its electrical properties but also for its mechanical and optical properties in many applications such as Micro Electro-Mechanical Systems (MEMS) and Nano Electro Mechanical System (NEMS), solar cells, photoelectrons, biomedical devices, waveguides and thermoelectric devices [1-10]. A thin film of silicon oxide can act as an etch stop and polish stop layer; also it is used as a passivation material which protects from chemical/mechanical damages $[1,2,6,10]$. The use of amorphorized thin-film silicon is one of the most important approaches in photovoltaic devices and solar cell fabrication $[11-13]$.
} 


\subsection{Fabrication Methods of Amorphorized/Oxidized Thin-Film}

In recent years, many methods have been developed to generate patterned oxidized/amorphorized silicon thin-film directly from the surface of bulk silicon substrates. Dependent on the size of the thin film, these methods can be characterized into two classes: techniques for large area oxidization/amorphorization and those for micro/nano scale oxidization/amorphorization. All of these techniques entail multiple time-consuming processing steps and employ complex and expensive equipments that need well-trained users for their operation.

\subsubsection{Chemical Vapor Deposition (CVD): large area oxidization/ amorphization}

In this process, all materials for depositing thin-film are supplied by an external source: additive materials or reactants. The reactant in a CVD process starts out in the vapor phase. The solid thin-film is deposited on the wafer surface through a chemical reaction of the reactant with oxygen. For example, the following reaction is used for the deposition of silicon oxide thin films.

$$
\mathrm{SiH}_{4}+\mathrm{O}_{2}+Q(\text { heat }) \rightarrow \mathrm{SiO}_{2}+2 \mathrm{H}_{2}
$$

There are different types of production systems for the oxidized/amorphorized silicon layer 
as follows:

- Atmospheric Pressure CVD (APCVD)

- Low Pressure CVD (LPCVD)

- Plasma -Assisted CVD (Plasma Enhanced CVD (PECVD) and High Density Plasma $C V D(\mathrm{HDPCVD}))$

APCVD is most commonly used for the deposition of oxidized silicon when it is to be used for interlayer dielectric layers. In this process, silicon oxide deposition can be done by oxidizing silane $\left(\mathrm{SiH}_{4}\right)$ with oxygen (as shown in Equation 1.1) or by reacting tetraethyl orthosilicate $\left(\mathrm{Si}_{2}\left(\mathrm{C}_{2} \mathrm{H}_{5} \mathrm{O}\right)_{4}\right)$ (TetraEthyl OrthosSilicate (TEOS)) with ozone.

$$
\mathrm{Si}\left(\mathrm{C}_{2} \mathrm{H}_{5} \mathrm{O}\right)_{4}+8 \mathrm{O}_{3} \rightarrow \mathrm{SiO}_{2}+10 \mathrm{H}_{2} \mathrm{O}+8 \mathrm{CO}_{2}
$$

LPCVD systems are more commonly used than APCVD for the deposition of silicon oxide due to their lower cost, higher production throughput, and superior film properties [14]. Silicon oxide deposition can be accomplished by decomposition of TEOS with or without oxygen at a temperature between $650^{\circ}-750^{\circ} \mathrm{C}$ or by oxidizing silane at the lower temperature of $450{ }^{\circ} \mathrm{C}$.

In the PECVD method, silicon oxide film is usually formed by reacting silane with oxygen or nitrous oxide $\left(\mathrm{N}_{2} \mathrm{O}\right)$ in a plasma with a temperature of $350^{\circ} \mathrm{C}$. Nitrous oxide gas can generate more uniform film than oxygen. 


$$
\mathrm{SiH}_{4}+2 \mathrm{~N}_{2} \mathrm{O} \rightarrow \mathrm{SiO}_{2}+2 \mathrm{~N}_{2}+2 \mathrm{H}_{2}
$$

Types of CVD methods and their principal characteristics are summarized in Table 1.1:

\begin{tabular}{|l|l|l|}
\hline Process & Advantages & Disadvantages \\
\hline APCVD & $\begin{array}{l}\text { Simple, fast and low tem- } \\
\text { perature }\end{array}$ & $\begin{array}{l}\text { Poor quality (step coverage), low } \\
\text { throughput and particle contamina- } \\
\text { tion }\end{array}$ \\
\hline LPCVD & $\begin{array}{l}\text { Good purity and uniform- } \\
\text { ity, large wafer capacity }\end{array}$ & $\begin{array}{l}\text { High temperature, low deposition } \\
\text { rate requires vacuum systems }\end{array}$ \\
\hline PECVD & $\begin{array}{l}\text { Low temperature, fast de- } \\
\text { position, good quality }\end{array}$ & $\begin{array}{l}\text { High cost and particle contamina- } \\
\text { tion }\end{array}$ \\
\hline
\end{tabular}

Table 1.1: Type of CVD methods and their principal characteristics

\subsubsection{Patterning of amorphorized-oxidized thin-film silicon in mi- cro/nano scale}

Several approaches have been proposed for the micro/nano scale patterning of amorphorized/oxidized silicon [15-29], among which techniques based on atomic force microscopy are the most widely investigated. These techniques include scanning probe oxidation [15,18-26], Tribo Nano Lithography (TNL) [27-29] and Dip-Pen Lithography (DPL) [25]. A common characteristic of these techniques is that they all involve two steps: first, the scanning probe either deposits or directly induces a thin layer of etch stop on the top surface of a silicon substrate; then the following chemical etching reveals the positive or negative features. In the scanning probe oxidation approach and the tribo nanolithography approach, the probe has to be in contact with the silicon substrate in order to generate an oxide or amorphous layer. 
Normally, a blunt-tipped probe will be employed and a mechanical force in the range of $\mu N$ must be applied to obtain an amorphorized or oxidized layer of thickness in sub-10 $\mathrm{nm}$ range. Due to the contact nature of these approaches, the minimum line width is limited by the dimension of the Atomic Force Microscope (AFM) tip, usually on the order of $50 \mathrm{~nm}$. In the case of dip pen nanolithography, line width of $5-15 \mathrm{~nm}$ can be achieved since a sharper tip can be employed for deposition. For these applications, the velocity of an AFM scanning probe is normally set to be around $300 \mathrm{~nm} / \mathrm{sec}$ and the scanning field $(X \times Y)$ of an AFM is typically around $100 \mu m \times 100 \mu m$. Thus, it is determined that nanolithography approaches that employ AFM are slow processes and only applicable to micron-sized devices. For large scale manufacturing, cell stitching must be performed and it could be very challenging due to the small dimension of each cell [15].

The following sections will give a more detailed description of the aforementioned scanning probe based methods.

\subsubsection{Scanning probe oxidation of silicon}

In this technique, the Scanning Transmission Microscopy (STM)/AFM tip is used to fabricate a thin layer of silicon oxide (directly grown from the top surface of a silicon substrate). Figure 1.1 illustrates the oxidation process [22]. In this technique, the AFM tip is used as a cathode and a positive bias voltage is applied to the silicon substrate.

This technique enables us to obtain high-resolution oxidized silicon nanopatterns. The line width and aspect ratio can be controlled by parameters such as tip bias, humidity of experimental environment, scanning speed, tip type and dimension of tip [22]. 
Using the generated oxidized silicon nanopatterns on a silicon surface as etching masks, wet alkaline chemical etching is used to generate nano features on a silicon substrate [22].
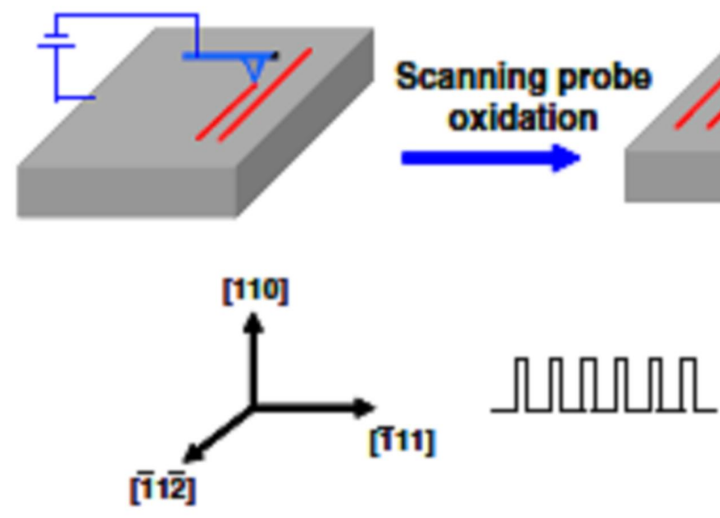

$\mathrm{Si}(110)$
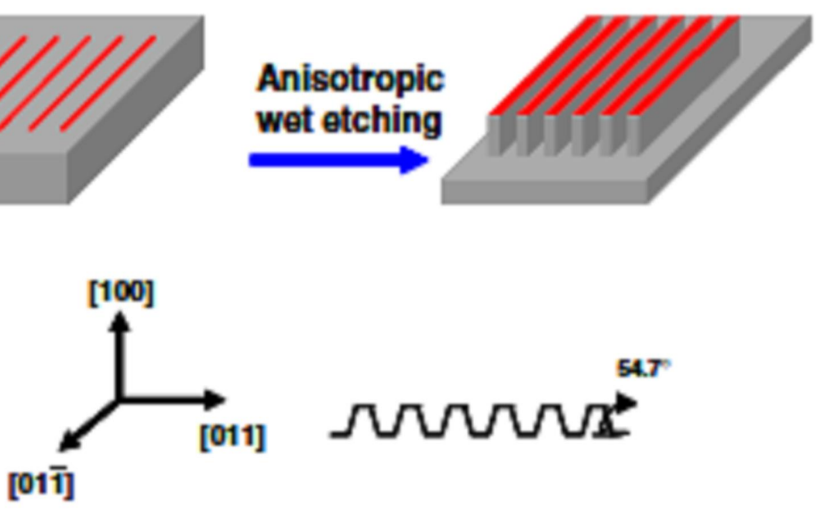

$\mathrm{Si}(\mathbf{1 0 0 )}$

Figure 1.1: Process of scanning probe oxidation of silicon [22]

\subsubsection{Tribo-nanolithography}

N Kawasegi et al [27], have proposed a new method for nanofabrication by combination of tribo-nanolithography and chemical etching based of atomic force microscopy (AFM). By using the TNL technique, a thin layer of predetermined pattern of amorphorized or oxidized silicon can be fabricated on top of the silicon substrate, which can function as an etch stop in alkaline etching solution such as Potassium Hydroxide (KOH). Figure 1.2 shows the fabrication process. Initially, a thin layer of amorphorized or oxidized silicon is generated on the silicon surface, then the sample is etched in $\mathrm{KOH}$ and finally micro/sub micro features are generated on top of the silicon substrate. 


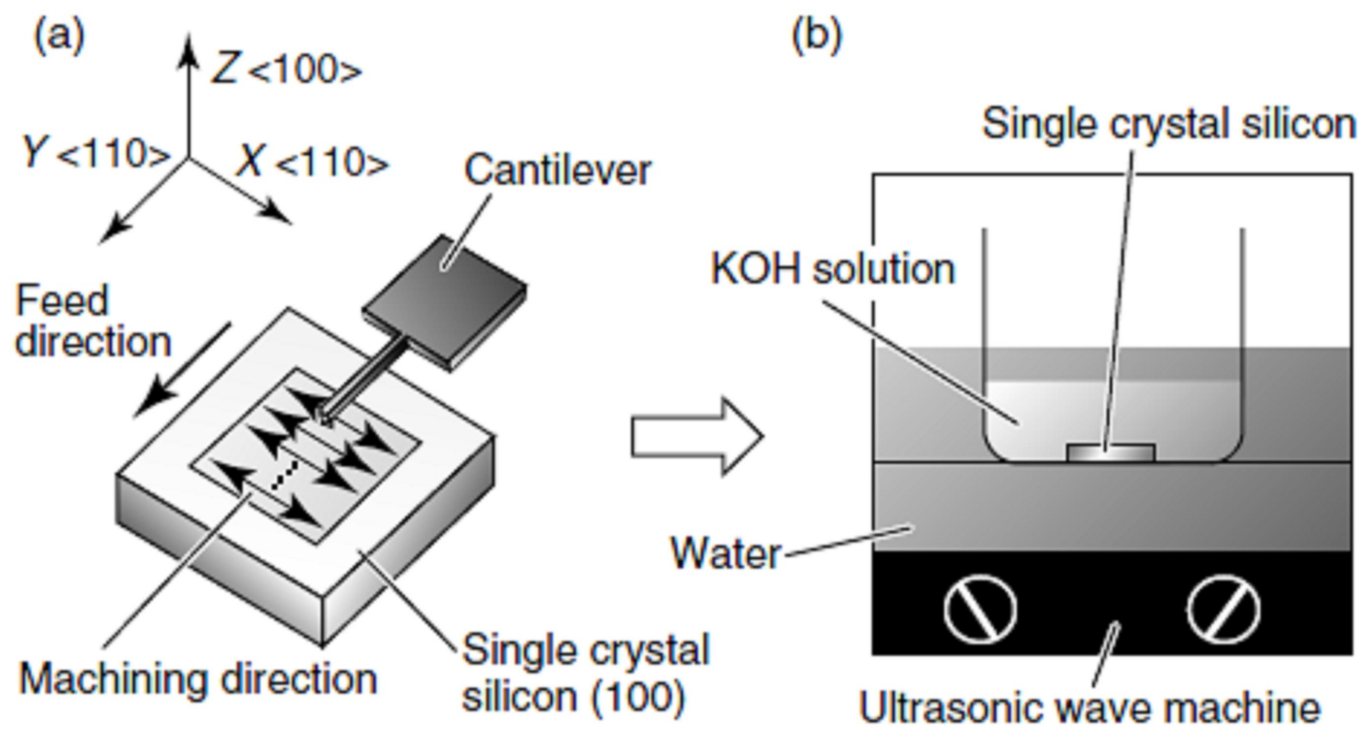

Figure 1.2: Process of Tribo-nanolithography of Si [27]

\subsubsection{Dip-pen lithography}

The AFM tip oxidation works on the top-down approach, recently, a top-up approach technique has been reported. Dip-Pen Nanolithography (DPN) utilizes an AFM to transport molecule-thick nanostructure from the tip to the $S i$ substrate surface via water meniscus and at ambient conditions. It has been reported that the DPN technique can offer $15 \mathrm{~nm}$ line widths and $5 \mathrm{~nm}$ spatial resolution. This method has been demonstrated for fabrication of pressure sensors, actuators, micro-optical component and mask used for micro/nano lithography techniques $[18,25]$. 


\subsection{Laser Processing of Silicon}

Laser processing of silicon $\mathrm{Si}$ ) has attracted important attention in the last decades. In the past, one of the interests in laser processing of $S i$ was the "laser annealing" process.

Laser annealing of $S i$ has some advantages over furnace annealing, including extended solid solubility of dopants in $S i$ and fewer defects after annealing. This improves electrical properties; hence the performance of the junction subjected to ion implantation [30].

The phase transformation processes by laser on $S i$ in the laser annealing process is one of the studies which became very attention-grabbing in the seventies. Recently, ultrashort laser-induced crystallization of amorphous silicon has been performed [31]. It is shown that polycrystalline silicon grains on the oxidized silicon wafer deposited by low-pressure chemical vapor deposition (LPCVD) could be obtained by scanning femtosecond laser pulses over the sample.

Amorphization of silicon was also reported during the ablation process of crystalline silicon induced with femtosecond laser pulses [32]. Cross-sectional Transmission Electron Microscopy (TEM) images showed a thin layer of amorphous silicon around the ablated holes. It is worth mentioning this study was in contrast with the hypotheses of direct solid-vapor transition [33] and negligible hydrodynamic motion (assumed on femtosecond laser ablation) [32].

One of the novel applications in using the femtosecond $(f s)$ laser is the oxidizing process. In the last decade, many interests have focused on the laser oxidation with potential applications in industry and in medicine [4-9,34-36]. Osipov et al. [35], reported the growth of silicon-dioxide thin-films $(<6 \mathrm{~nm})$ on silicon surfaces in a dry oxygen atmosphere $(0.1-10$ 
Pa) at low temperatures $\left(35-200{ }^{\circ} \mathrm{C}\right.$. The oxidation process was induced by laser pulses with the pulse duration of $157 \mathrm{~nm}$. The kinetics of low-temperature oxidation was similar for the Si surfaces investigated and differs from that of high-temperature thermal oxidation $\left(900-1200{ }^{\circ} \mathrm{C}\right)$ that can be described by the Deal-Grove model [37]. The oxidation occurs by diffusion of oxygen atoms $O$ and/or ions $O^{-}$. rather than oxygen molecules. It was reported by [6], that the oxidation process can be controlled by the laser beam energy density rather than by the surface temperature and a higher laser intensity results in a thicker oxidized layer. However, all these methods can be used only for the fabrication of large area oxidation; for micro/nano patterning of silicon oxide, these methods should be combined with more techniques such as lithography and etching processes.

Upon laser beam irradiation, a solid undergoes thermal heating. Dependent on laser parameters, the solid can be heated up to several regimes, each associated with characteristic phenomena and the material breakdown/modification mechanism. With high laser intensity, ablation occurs and a solid will be removed. With lower laser intensity, surface modification happens, where properties and morphology of the top surface of a solid will be altered without material break-down. Moreover, dependent on laser parameters, several types of surface modification could occur, including oxidation and amorphization. Solid heating under laser irradiation is complex and usually involves several material breakdown/modification mechanisms simultaneously. The main challenge of laser microscale silicon annealing is to obtain pure silicon oxide or amorphorized silicon $(a-S i)$ free from other laser irradiation induced products. To the best of author's knowledge, there is no reported work on laser micro/nano oxidation/amorphiozation patterning of silicon. 


\subsection{Objectives}

The recent advancement in high repetition rate femtosecond lasers has opened up new possibilities. Unlike the conventional femtosecond laser, the high repetition rate femtosecond laser delivers high average power by nanojoule pulses emitted at mega hertz frequency. The nanojoule pulse energy allows for precise control of thermal heating, hence delicate material modification. The high average power guarantees acceptable fabrication efficiency. Therefore, a layer of silicon oxide or amorphorized silicon may be selectively generated on the silicon substrate using a megahertz femtosecond laser. Therefore, the main objective is to study the high repetition femtosecond laser pulse silicon annealing mechanism through five individual but related tasks:

- Conceptual development of direct Silicon oxidation induced with high repetition ultrashort laser pulses;

- Conceptual development of direct Silicon amorphization induced with high repetition ultrashort laser pulses;

- Study the effect of laser parameters on laser annealing (modification) process;

- Demonstration of application of direct amorphization and oxidization for micro/nano scale lithography;

- Demonstration of application of direct amorphization and oxidization for solarcell fabrication; 


\section{Chapter 2}

\section{Physics of Laser Annealing of Silicon}

\subsection{Properties of Silicon}

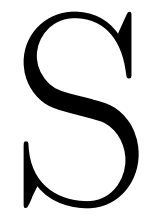

ILICON plays a crucial role in the fabrication of nano and micro electromechanical systems (NEMS, MEMS) $[4-6,36]$. It is used extensively in producing conductor, semiconductor, solar cell and Lab-On-a-Chip (LOC) systems [7-9].

The energy gap of the silicon is $1.12 \mathrm{eV}$ which causes silicon devices to be less prone to thermal noise than germanium devices with $0.67 \mathrm{eV}$ [10]. Silicon is a semiconductor material which is available in both p-type (holes as charge carriers) and n-type (electron charge carriers). The resistivity of the silicon can vary in a wide range from 0.001 to $20000 \mathrm{ohm}-\mathrm{cm}$. Silicon wafers are available in various diameters $(100,125, \ldots, 300, \ldots)$ and thicknesses in different crystal orientations with the ability to control the crystal properties in the fabrication process. Furthermore, silicon is a strong material which many devices such as 
cantilevers, membranes, sensors, resonators and other mechanical/electromechanical devices are made from. In fact, micro/nano electromechanical (MEMS and NEMS) devices utilize the excellent semiconductor and mechanical properties of silicon [10].

As shown in Figure 2.1, silicon and its compounds can be found in the following forms [10]:

- Single crystalline (monocrystalline)

- Poly-crystalline

- Amorphous $(a-S i)$
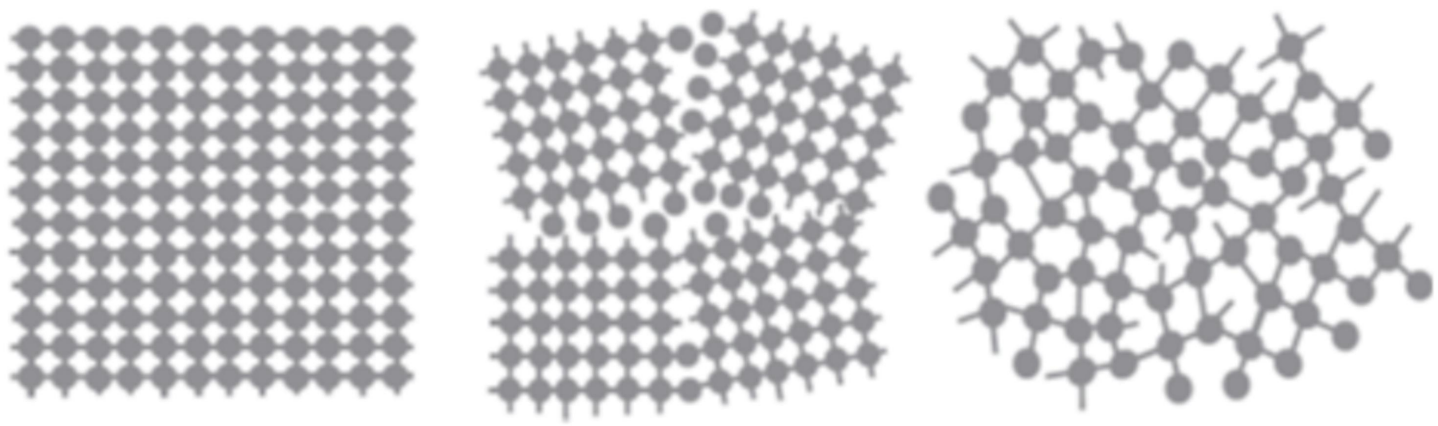

Figure 2.1: Different Si forms (crystalline, multicrystalline and amorphous) [14]

The simplest arrangement of atoms is called the unit cell, which gives the crystal structure when repeated in a three-dimensional framework. If the unit cells are in a regular arrangement and have a repeatable manner, then the crystal structure is called monocrystal or single-crystal. In order to have the desirable electrical and mechanical properties, mono crystalline silicon structure (single crystal) is used in semiconductor wafer processing [14]. If the unit cells of the material are not in a repetitive (regular) arrangement, as shown in Figure 2.1, the material is in polycrystalline form. Another name for polycrystalline silicon 
is polysilicon [14]. An analogy for polycrystalline silicon is a pile of bricks.

Amorphorized materials are the opposite of crystalline materials that lack a repetitive structure and demonstrate structural disorder as shown in Figure 2.1. Plastic is an example of an amorphous material. Amorphous silicon, as discussed later, is an insulator material which has attracted a lot of attention for solar cell fabrication. Moreover, it has some chemical/physical properties which make it suitable for different industrial applications such as photolithography $[10,14]$.

\subsection{Laser Annealing of Silicon}

\subsubsection{Irradiation of a solid target by a single laser pulse}

The fundamental physical mechanism of laser annealing of silicon is governed by the amount of energy transferred from the electron-hole plasma to the irradiated surface in the time scale of pulse duration [38-41]. Therefore, pulse duration plays a crucial role in laser processing. Generally, shorter pulse duration allows for energy to be deposited in a very short time span; therefore, extremely high power can be achieved. For this reason lasers of shorter pulse are preferred for material processing.

The first lasers, invented in 1960 [42], based on a ruby crystal and pumped by a xenon flash discharge, had pulse durations in the range of millisecond to microsecond. Introducing modern laser systems such a Q-switching and mode locking has reduced the pulse duration even to nanosecond $\left(1 \mathrm{~ns}=10^{-9} \mathrm{~s}\right)$, picosecond $\left(1 \mathrm{ps}=10^{-12} \mathrm{~s}\right)$, femtosecond $\left(1 \mathrm{fs}=10^{-15} \mathrm{~s}\right)$ 
and recently attosecond $\left(1 a s=10^{-18} s\right)$ duration [43] . Using ultrashort or ultrafast pulses, new subfields of science have been created, namely femtochemistry $[44,45]$ and femtobiology [46] in which the phenomena could be resolved on ultrashort time-scale resolution.

Many approaches have been carried out on the $n s$ laser pulses for the laser annealing process. This $n s$ laser pulse energy fluence is high enough for melting a surface layer of the amorphous $S i$ created by the ion implantation of dopants on single crystalline silicon. Resolidification of the molten silicon removes the lattice damage and electrically activates the dopants with free defects. Nanosecond laser pulses are used for recrystallizing the fine-grained polycrystalline silicon layer deposited on the insulating substrate, for example, silicon-on-sapphire [30]. Using the picosecond laser for heating the material substrate, heating and cooling rates up to $10^{14}{ }^{\circ} \mathrm{C} / \mathrm{s}$ are obtained [47]. Due to this high cooling rate, the kinetics of phase transitions leads to a new phenomenon. In fact, by controlling the energy density of the Gaussian laser pulse, amorphization of crystalline silicon or recrystallization of this amorphous layer can be achieved [47].

The energy which is transferred into the silicon lattice causes the silicon surface to melt. It results in increasing the optical absorption of the irradiated substrate; thus, the molten layer of silicon is heated further and the absorbed energy propagates deeper into the silicon. At the end of the laser pulse (between pulses), no more energy is supplied to the silicon substrate and the absorbed heat diffuses to the cooler surrounding. Finally recrystallization of the silicon occurs. However, if the absorbed energy is not sufficient for recrystallization, or due to the high cooling rate (in case of using Pico/femtosecond laser pulses), re-solidification occurs at randomly distributed sites, which results in the amorphization or poly-crystallization rather than single crystal materials $[15,47]$. 
Numerical analysis of surface temperature after laser irradiation is rather complicated. To start with, it is considered a simple case of the surface irradiated by a single pulse where irradiation and plume expansion losses can be neglected. It is also assumed that the incident laser fluence is absorbed in a thin layer of surface in accordance with the one-dimensional heat conduction equation [39].

$$
\frac{\partial T}{\partial t}=a \frac{\partial^{2} T}{\partial x^{2}}
$$

where, $a=\frac{k}{C_{p} \rho_{0}}$.

Here, $a$ is the thermal diffusion coefficient, $k$ is the heat conduction coefficient $C_{p}$ is specific heat, $\rho_{0}$ is target material density, $t$ is time and $x$ is the space-dimension.

Assuming the laser pulse intensity profile in time has a rectangular shape, it can be concluded:

$$
T(x, t)=\frac{a^{1 / 2}}{k \pi^{1 / 2}} \int_{0}^{t_{p}} \frac{I_{a}(\tau)}{t-\tau} \exp \left\{-\frac{x^{2}}{2 a(t-\tau)}\right\} d \tau
$$

Thus, the average temperature as a function of the laser and target parameters can be obtained by:

$$
\langle T\rangle=\frac{1}{\left(a t_{p}\right)^{1 / 2}} \int_{0}^{\infty} T\left(x, t_{p}\right) d x .
$$

For the rectangular shaped pulse, the value of the average temperature as a function of the laser and target parameters can be obtained in an explicit form as: 


$$
\langle T\rangle=\left(\frac{2}{\pi}\right)^{1 / 2} \frac{I_{a}\left(a t_{p}\right)^{1 / 2}}{k} \frac{I_{a}\left(a t_{p}\right)^{1 / 2}}{k}=\frac{1}{2^{1 / 2}} T\left(0, t_{p}\right) .
$$

The maximum surface temperature occurs at the end of laser pulse and at the center of ablation ( $\mathrm{x}=0, t=t_{p}$, where, $t_{p}$ is pulse duration). Thus, the target temperature during the laser pulse can be calculated by:

$$
T(t)=T_{m}\left(\frac{t}{t_{p}}\right)^{1 / 2}
$$

Finally, the time dependence of the target temperature after the end of the laser pulse is given by [39]:

$$
T=(0, t)=T\left(0, t_{p}\right)\left(\frac{t_{p}}{t}\right)^{1 / 2}=\sqrt{\frac{2}{\pi}} \frac{I_{a}\left(a t_{p}\right)^{1 / 2}}{k}\left(\frac{t_{p}}{t}\right)^{1 / 2}
$$

\subsubsection{Surface temperature of the target in the case of a succession of laser pulses}

With the short pulsed laser, it is more common to use a train of pulses than a single pulse for material removal or modification. Therefore, the aforementioned one-dimension heat transfer model must be extended to include the effect of heat accumulation due to the succession of pulses.

The process of heating the target surface by the succession of laser pulses with the pulse 
duration of $t_{p}$ followed by time intervals $t_{p p}\left(t_{p p}=1 / f\right)$ can be explained as follows [39]:

$$
T(t)=T_{\max }\left(t / t_{p p}\right)^{1 / 2}
$$

The relation between the maximum surface temperature at the end of the first pulse, $T_{\max }$ , and the surface temperature at the beginning of the following laser pulse, $T_{\min }$, is given by [39]:

$$
T_{\min }=T_{\max }(t p / t p p)^{1 / 2}
$$

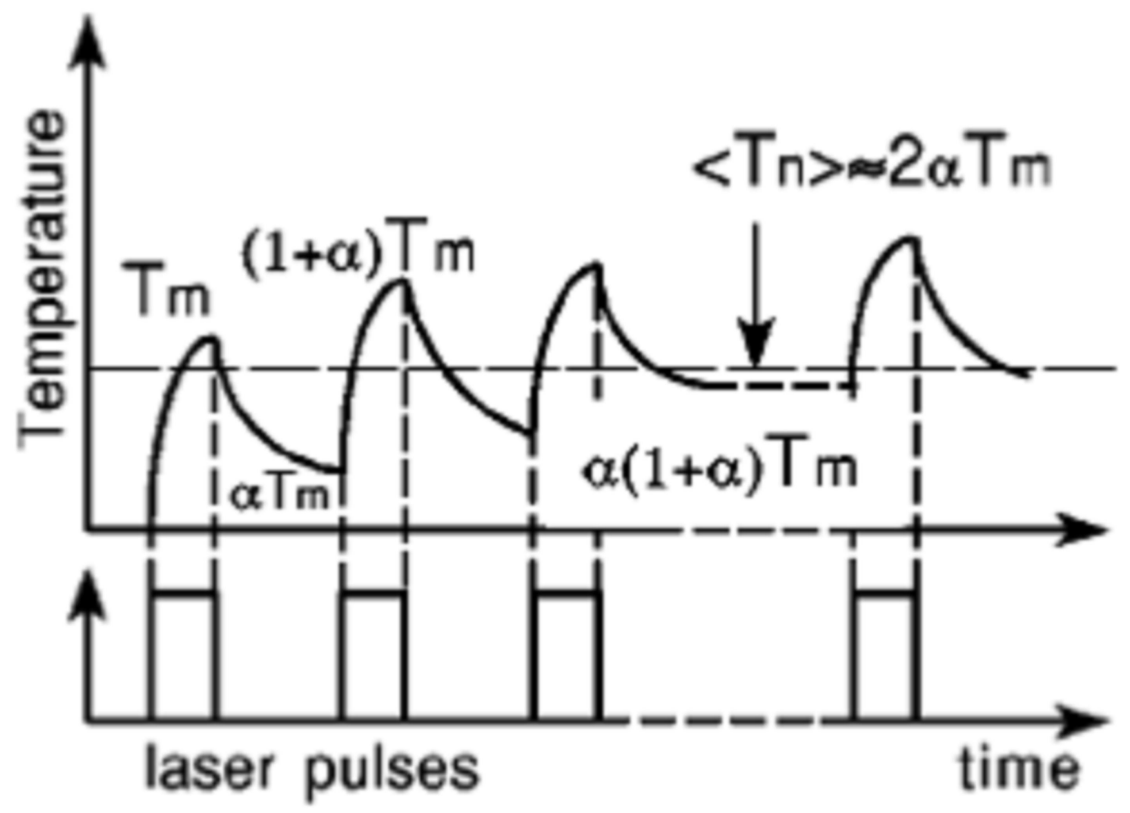

Figure 2.2: Temperature of the target surface heated by a series of laser pulses [39]

Thus, the maximum and minimum surface temperature of the surface after " $n$ " number of laser pulses can be easily calculated by: 
First Pulse:

$$
\begin{aligned}
& \left(T_{\max }\right)_{1}=T_{m} \\
& \left(T_{\min }\right)_{1}=\alpha T_{m}
\end{aligned}
$$

Second Pulse:

$$
\begin{aligned}
& \left(T_{\min }\right)_{2}=\alpha(1+\alpha) T_{m} \\
& \left(T_{\max }\right)_{2}=(1+\alpha) T_{m}
\end{aligned}
$$

$n$th pulse:

$$
\begin{aligned}
& \left(T_{\max }\right)=\left(1+\alpha+\alpha^{2}+\alpha^{3}+\ldots+\alpha^{n-1}\right) T_{m}=\left[\left(1-\alpha^{n}\right) /(1-\alpha)\right] T_{m} \\
& \approx(1-\alpha)^{-1} T_{m}\left(T_{\min }\right)_{n}=\alpha\left(T_{\max }\right)_{n}
\end{aligned}
$$

The average surface temperature by time over the interaction time is:

$$
\bar{T}=\frac{1}{t} \int T(0, t) d t .
$$

The average surface temperature over any $i$ th laser pulse and the time gap between $i$ th and $(i+1)$ th pulses, is calculated by:

$$
\bar{T}_{i}=\frac{1}{t_{p}+t_{p p}} \int_{0}^{t_{p}+t_{p p}} T_{m, i}(0, t) d t=2 \alpha T_{m, i} \frac{1-\frac{2}{3} \alpha}{1+\alpha^{2}}
$$

The average surface temperature after " $n$ " pulses [39]:

$$
\bar{T}_{n}=\frac{1}{n} \sum_{i=1}^{n} \overline{T_{i}}
$$




$$
\bar{T}_{n}=\frac{1}{n\left(t_{p}+t_{p p}\right)} \int_{0}^{n\left(t_{p}+t_{p p}\right.} T(0, t) d t
$$

where,

$$
\sum_{i=1}^{n} \bar{T}_{\max , i}=T_{m}\left(1+\frac{1-\alpha^{2}}{1-\alpha}+\ldots+\frac{1-\alpha^{n}}{1-\alpha}\right)=\frac{T_{m}}{(1-\alpha)}\left(n+\frac{\alpha^{n}-\alpha}{1-\alpha}\right)
$$

The final form of average surface temperature can be obtained as follows:

$$
\bar{T}_{n}=2 \alpha \frac{\left(1-\frac{2}{3} \alpha\right)}{\left(1+\alpha^{2}\right)} \cdot \frac{T_{m}}{(1-\alpha)}\left(1+\frac{\alpha^{n}-\alpha}{n(1-\alpha)}\right) .
$$

In the case $n \gg 1$ and $\alpha \ll 1$

$$
\bar{T}_{n} \cong 2 \alpha T_{m}=2 T_{m}\left(\frac{t_{p}}{t_{p p}}\right)^{1 / 2}
$$

It can be concluded that the increase in laser frequency results in the increase in pulse interval and hence results in the increase of the surface temperature. The heating process of laser irradiation and the effect of the laser parameters will be discussed in the following chapters. 


\subsubsection{Non-dimensional surface temperature of laser irradiation and effect of laser parameters}

The heat conduction for cylindrically symmetric flow with no internal heat generation is given as [48]:

$$
\frac{1}{r} \frac{\partial}{\partial r}\left(k r \frac{\partial T}{\partial r}\right)+\frac{\partial}{\partial z}\left(k \frac{\partial T}{\partial z}\right)=\rho C_{p} \frac{\partial T}{\partial t},
$$

For isotropic material properties:

$$
\frac{1}{r} \frac{\partial}{\partial r}\left(r \frac{\partial T}{\partial r}\right)+\frac{\partial^{2}}{\partial z^{2}}=\frac{1}{\alpha} \frac{\partial T}{\partial t},
$$

The absorbed heat from a beam with a Gaussian distribution can be written using Lambert's law as follows:

$$
I(r, z)=I_{0} \exp (-\delta z) \exp \left(-r^{2} / r_{0}^{2}\right),
$$

It can be assumed there is no heat loss. [48]:

$$
\left.\frac{\partial T}{\partial z}\right|_{z=0}=-\frac{1}{k} \frac{\eta P P(t)}{\pi r_{0}^{2}} \exp \left(-\frac{r^{2}}{r_{0}^{2}}\left(t \preceq t_{p}\right),\right.
$$

where, $P P$ is the laser peak power, $h$ is the fraction of the absorbed surface energy, and $r_{0}$ is the beam diameter. 
Because there are a wide range of scales among the variables of interest, it is more convenient to introduce the nondimensional groups as follows [49]:

$$
\begin{aligned}
& R=r / r_{0}, \\
& Z=z / r_{0}, \\
& \Theta=T / T_{\text {ref }}, \\
& \tau=4 \alpha t / r_{0}^{2}
\end{aligned}
$$

Thus,

$$
\frac{1}{R} \frac{\partial}{\partial R}\left(R \frac{\partial \Theta}{\partial R}\right)+\frac{\partial^{2} \Theta}{\partial Z^{2}}=4 \frac{\partial \Theta}{\partial \tau}
$$

With the boundary condition given by

$$
\left.\frac{\partial \Theta}{\partial Z}\right|_{Z=0}=-Q \exp \left(-R^{2}\right)\left[u(\tau)-u\left(\tau-\tau_{p}\right)\right]
$$

Here, $Q$ is the nondimensional power $\left(Q=h P P / k p r_{0} T_{r e f}\right)$ and $\mathrm{u}(\mathrm{t})$ is the unit step function. Moreover, it is assumed that the absorption coefficient and peak power are constant.

The temperature for $\tau \geq \tau_{p}$ in integral form is given by [48]:

$$
\Theta(R, Z, \tau)=\frac{Q}{2 \sqrt{\pi}} \int_{\tau-\tau_{p}}^{\tau} I(\nu) d \nu
$$

where, 


$$
I(\nu)=\left(\frac{1}{1+\nu}\right) \frac{1}{\sqrt{\nu}} \exp \left(-\frac{R^{2}}{1+\nu}-\frac{Z^{2}}{\nu}\right)
$$

The maximum temperature at the center of the beam is calculated by [49]:

$$
\Theta_{\text {max }}(\tau)=\frac{Q}{\sqrt{\pi}}\left(\arctan (\sqrt{\tau})-\arctan \left(\sqrt{\tau-\tau_{p}}\right)\right) .
$$

For short pulse times [50]:

$$
\Theta(0,0, \tau)=\frac{Q}{\sqrt{\tau}} \quad\left(\tau<\tau_{p}\right)
$$

For longer pulse times the maximum temperature can be calculated by: $Q_{\max }=Q \sqrt{\pi} / 2$ For steady state and using the same boundary conditions:

$$
\Theta_{s s}(R, Z)=\frac{Q}{2} \int_{0}^{\infty} \exp (-\lambda Z) \exp \left(-\lambda^{2} / 4\right) J_{0}(\lambda R) d \lambda,
$$

Here, $J_{0}$ is the Bessel function of the first kind of order zero.

Thus, the series solution in integral form in given by $[48,49]$ :

$$
\Theta_{s s}(R, Z)=\frac{Q \sqrt{\pi}}{2} \sum_{n=0}^{\infty}(-1)^{n} R^{2 n} \frac{(2 n) !}{(n !)^{2}} \times \exp \left(Z^{2}\right) i^{2 n} \operatorname{erf} c(Z) .
$$

Equation 2.31 can be simplified to: 


$$
\begin{gathered}
\Theta_{s s}(R=0, Z)=\frac{Q \sqrt{\pi}}{2} \exp \left(Z^{2}\right) \operatorname{erfc}(Z) \\
\Theta_{s s}(R, Z=0)=\frac{Q \sqrt{\pi}}{2} \exp \left(-R^{2} / 2\right) I_{0}\left(R^{2} / 2\right)
\end{gathered}
$$

The non-dimensional temperature can be given as follows:

$$
\Theta(R, Z, \tau)=\frac{Q}{2 \sqrt{\pi}}\left(\int_{0}^{\tau} I(\nu) d \nu-\int_{0}^{\tau_{\tau} p} I(\nu) d \nu\right)
$$

where, $I(\nu)$ is:

$$
I(\nu)=\left(\frac{1}{1+\nu}\right) \frac{1}{\sqrt{\nu}} \exp \left(-\frac{R^{2}}{1+\nu}-\frac{Z^{2}}{\nu}\right)
$$

For short time solution, $t<1$ :

$$
\begin{aligned}
& \int_{0}^{\tau}\left(\frac{1}{1+\nu}\right) \frac{1}{\sqrt{\nu}} \exp \left(-\frac{R^{2}}{1+\nu}-\frac{Z^{2}}{\nu}\right) d \nu \\
& =\sum_{n=0}^{\infty} e^{-R^{2}}(-1)^{n} L_{n}\left(R^{2}\right) \Gamma\left(-n-\frac{1}{2}\right) \times\left[Z^{2 n+1}-\tau^{n+\frac{1}{2}} \gamma^{\star}\left(-n-\frac{1}{2}, \frac{Z^{2}}{\tau}\right)\right]
\end{aligned}
$$

where, $L_{n}$ are the Laguerre polynomials: 


$$
L_{n}(x)=\frac{e^{x}}{n !} \frac{d^{n}}{d x^{n}}\left(x^{n} e^{-x}\right) .
$$

$\Gamma_{x}$ is the gamma function:

$$
\Gamma\left(-n-\frac{1}{2}\right)=\frac{(-2)^{n+1} \sqrt{\pi}}{(2 n+1) ! !}(n=0,1, \ldots)
$$

And, $\gamma^{\star}$ is a form of the incomplete gamma function:

$$
\gamma^{\star}(a, x)=\frac{x^{-a}}{\Gamma(a)} \int_{0}^{x} e^{-t} t^{a-1} d t .
$$

For long time solution $(\tau>1)$

$$
\begin{gathered}
\int_{0}^{\tau} I(\nu) d \nu=\int_{0}^{\infty} I(\nu) d \nu-\int_{\tau}^{\infty} I(\nu) d \nu . \\
\int_{\tau}^{\infty} I(\nu) d \nu=\Sigma_{n=0}^{\infty} L_{n}\left(-R^{2}\right)(-1)^{n} \frac{\Gamma\left(n+\frac{1}{2}\right)}{\tau^{n+\frac{1}{2}}} \times \gamma^{\star}\left(n+\frac{1}{2}, \frac{Z^{2}}{\tau}\right) .
\end{gathered}
$$

The maximum surface temperature, which occurs at the end of the laser pulse on the substrate can be approximately calculated by:

$$
\theta\left(R, Z=0, \tau_{p}\right) \approx \frac{Q}{\sqrt{\pi}} \arctan \left(\sqrt{\tau_{p}}\right) \exp \left(-R^{2}\right)
$$




$$
\theta\left(R, Z=0, \tau_{p}\right) \approx \frac{Q}{\sqrt{\pi}} \arctan \left(\sqrt{\tau_{p}}\right)-Q Z
$$

The maximum surface temperature which occurs at the center of the laser beam is $[48,49]$ :

$$
\theta_{\text {max }}(\tau)=\frac{Q}{\sqrt{\pi}}\left[\arctan (\pi)-\arctan \left(\sqrt{\left.\tau-\tau_{p}\right)}\right]\right.
$$

For $\tau<\tau_{p}$ :

$$
\theta(0,0, \tau)=\frac{Q}{\sqrt{\pi}} \sqrt{\tau}
$$

In the following chapters, the developed formula will be used to study the surface temperature change induced by laser irradiation and the effect of the laser. 


\title{
Chapter 3
}

\section{Experimental Setup and Procedure}

\author{
7 HIS chapter details the experimental setup and procedures. The center piece \\ of equipment used for this research is a Mega Hertz femtosecond laser system \\ L located in the Micro and Nano Fabrication Research Laboratory at Ryerson \\ University, Toronto, Canada.
}

\subsection{Laser System}

The laser system used in this study is a diode-pumped Yb-doped fiber oscillator/amplifier system which is capable of producing variable frequency rates in the range of $200 \mathrm{kHz}$ to $26 \mathrm{M} \mathrm{Hz}$ with an average power output of $12 \mathrm{~W}$ at the frequency of $2 \mathrm{M} \mathrm{Hz}$ (model: ClarkMXR Inc. IMPULSE Series Ultrashort Pulse Laser). The pulse duration of the output laser can be adjusted from 10 picosecond to 200 femtosecond (min) using the computer. 
The Yb-doped fiber- oscillator /fiber-amplifier design allows for the solid sated low-noise performance to be combined with the fiber lasers high spatial mode quality. The central wavelength of the produced beam is $1030 \mathrm{~nm}[15,51]$.

All the laser parameters including repetition rate, pulse duration and average power are computer controlled. However, parameters such as pulse duration and frequency can not be varied continuously and only discrete variations are possible to be chosen by the user(characteristic of mode-lock laser systems).

\subsubsection{Optical Setup}

The experiments were carried out using two different optical setups for the amorphization and oxidation process. In the amorphization and oxidation process, laser pulses with wavelength of $1030 \mathrm{~nm}$ and $515 \mathrm{~nm}$ were used, respectively. The process of the direct oxidation with the femtosecond laser requires stringent parameter control; thus, laser pulses with wavelength of $515 \mathrm{~nm}$ were chosen for the oxidation process (due to less fluctuation in laser power).

\subsubsection{Amorphization Process}

Figure 3.1 shows the schematic drawing of the experimental setup. The diameter of the circular output beam from the laser is around $4.5 \mathrm{~mm}$. The laser beam was expanded to $9 \mathrm{~mm}$ by a combination of a plano-convex $(f=-100)$ and a plano-concave $(f=200)$ lenses; then the laser beam was rotated to circularly polarized by a quarter waveplate placed in the beam path. The diameter of the beam was reduced to $8 \mathrm{~mm}$ by using an iris diaphragm 
before entering to galvo scanner. A two-axis galvo-scanner was used for beam scanning since it has a high scanning speed (to $3000 \mathrm{~mm} / \mathrm{s}$ ). Such a high speed is very difficult to achieve with a mechanical translation stage. In order to focus the normal beam on the surface of silicon, the scan lens of a focal length of $63.5 \mathrm{~mm}$ was employed. The theoretical focused laser spot diameter $\left(d_{0}\right)$ is calculated from: $d_{0} \approx 1.27 \lambda_{0} F / D[15,52]$. Here, $F$ is the effective focal length of the scan lens, $\lambda_{0}$ is the wavelength of the laser equal to $1030 \mathrm{~nm}$ and $D$ is the laser beam diameter at the input of the galvo-scanner, which is $8 \mathrm{~mm}$. From this formula, the theoretical spot size is calculated to be $10.38 \mu m$ in diameter. During the experiment the spot size may be bigger due to scatter and misalignment. The average laser fluence was calculated to be $0.15 \sim 0.35 \mathrm{~J} / \mathrm{cm}^{2}$ at the repetition rate of 13 and $26 \mathrm{M} \mathrm{Hz}$. In the present experiment the dwell time is $0.1 \mathrm{~ms}$ and the scanning speed of line features was in the range of $100-150 \mathrm{~mm} / \mathrm{s}[15,53]$.

1. Modulator

2. $1030 \mathrm{~nm}$ Mirror

3. $\quad \mathrm{F}=-100 \mathrm{~mm}$ Concave Lens

4. $\quad \mathrm{F}=200 \mathrm{~mm}$ Convex Lens

5. $\quad 1030 \mathrm{~nm}$ Waveplate

6. $1030 \mathrm{~nm}$ Mirror

7. Diaphragm

8. Galvo-scanner

9. $\quad \mathrm{F}=63.5 \mathrm{~mm}$ Convex Lens

10. Silicon Wafer

11. X-Y Translation Stage

12. Computer

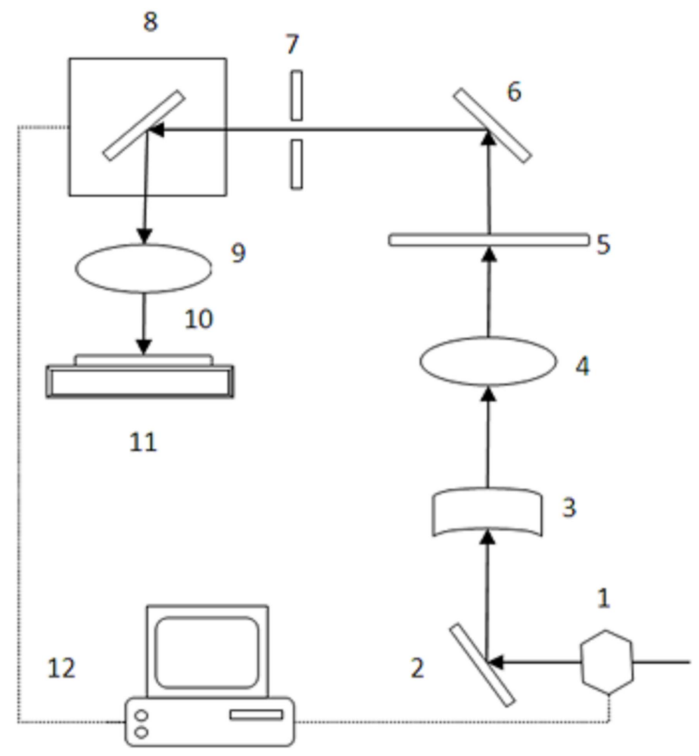

Figure 3.1: Experimental setup for amorphization process 


\subsubsection{Oxidation Process}

The optical setup used for oxidation is similar to the one described in Figure 3.1, except that a harmonic generator is needed to convert the wavelength. Accordingly, reflection mirrors shown in Figure 3.1 should be replaced by mirrors designed for $515 \mathrm{~nm}$.

Using a harmonic generator, the laser beam is frequency doubled from $1030 \mathrm{~nm}$ to $515 \mathrm{~nm}$. The theoretical diameter of the focused laser spot is calculated to be $1.02 \mu \mathrm{m}$. Samples were irradiated with laser beams of power ranging from $0.2 \mathrm{~W}$ to $1 \mathrm{~W}$ at pulse frequency ranging from $8 \mathrm{MHz}$ to $26 \mathrm{MHZ}(8,13$ and $26 \mathrm{MHz})$ and a speed of scanning of $500 \mathrm{~mm} / \mathrm{s}$. The schematic drawing of the experimental setup is shown in Figure $3.2[51,54]$.

\subsection{Samples, Procedure and Analyzing}

To study the direct patterning of silicon oxide and amorphorized silicon, undoped $<100>$ oriented silicon wafers were used. Prior to laser irradiation, silicon samples were rinsed in acetone and then treated in pure water. First, silicon wafers were irradiated of predetermined patterns with fs pulses with pulse energy controlled in oxidation or amorphization range under ambient conditions. In order to investigate the feasibility of using this technique in applications such as lithography, the irradiated samples were etched in a $\mathrm{KOH}$ solution (KOH $30 \%$, temperature: $\left.80^{\circ} \mathrm{C}\right)[15,54]$.

Irradiated and etched samples were examined by varies characterization tools. SEM was used to characterize the morphology; EDX spectroscopy, XRD and micro-Raman spectroscopy determined the chemical and material properties. Optical near field microscope and optical 
1. $\quad 1030 \mathrm{~nm}$ mirror

2. $\mathrm{F}=500 \mathrm{~mm}$ Convex Lens

3. $1030 \mathrm{~nm} \lambda / 2$ Waveplate

4. $\mathrm{F}=-150 \mathrm{~mm}$ Convex Lens

5. $\quad 515 \mathrm{~nm}$ Harmonic Generator

6. $\quad 515 \mathrm{~nm}$ Mirror

7. $\quad 515 \mathrm{~nm}$ Mirror

8. $515 \mathrm{~nm}$ Mirror

9. $\quad 515 \mathrm{~nm} \lambda / 4$ Waveplate

10. Diaphragm

11. Galvo scanner

12. $\mathrm{F}=63.5 \mathrm{~mm}$ Lens

13. Sample and Sample Holder

14. Modulator

15. Computer

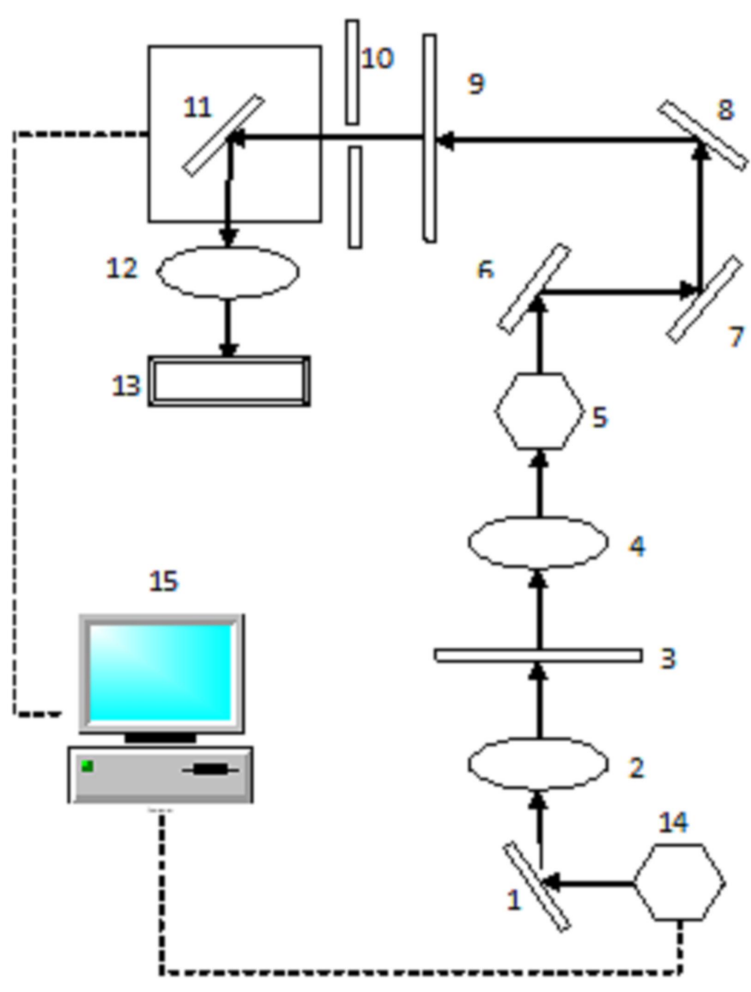

Figure 3.2: Experimental setup for oxidation process

spectrometer were used to evaluate the optical properties. Back scattering micro-Raman analysis was performed at room temperature using the $532 \mathrm{~nm}$ line of the $\mathrm{Ar}$ ion laser source $[15,51]$.

More explanation on how these techniques are used for studying the material/chemical/optical properties of samples, will be provided in following chapters. 


\section{Chapter 4}

\section{Direct-writing of Oxidized Silicon}

\section{Thin-film on Si Substrate}

\footnotetext{
HIS chapter reports the attempt of developing a new method for the generation of the silicon oxide pattern in micro-scale based on high frequency pulses of

L the femtosecond laser. The proposed approach enables a direct (single-step) generation of an oxide layer on a silicon substrate. In comparison with previous methods, the direct oxidation of silicon induced by the femtosecond laser is a maskless single-step technique which offers a higher flexibility and reduced processing time.
} 


\subsection{Experimental Results}

Although a wide range of laser power at various pulse frequencies was used for irradiation, micro scale embossed lines were only evident at two points: laser power of $1 \mathrm{~W}$ at a pulse frequency of $13 \mathrm{MHz}\left(9 \mathrm{~J} / \mathrm{cm}^{2}\right)$ and $0.35 \mathrm{~W}$ at $26 \mathrm{MHz}\left(1.65 \mathrm{~J} / \mathrm{cm}^{2}\right)$. In other instances, the irradiated area was either completely ablated or slightly modified. As shown in Figure 4.1, at $8 \mathrm{MHz}$, the line on the left presents no material breakdown but color-change, whereas at $26 \mathrm{MHz}$, the line on the right clearly shows material removal. The embossed line can only be generated at $1 \mathrm{~W}$ at $13 \mathrm{MHz}$ (middle line) [51].

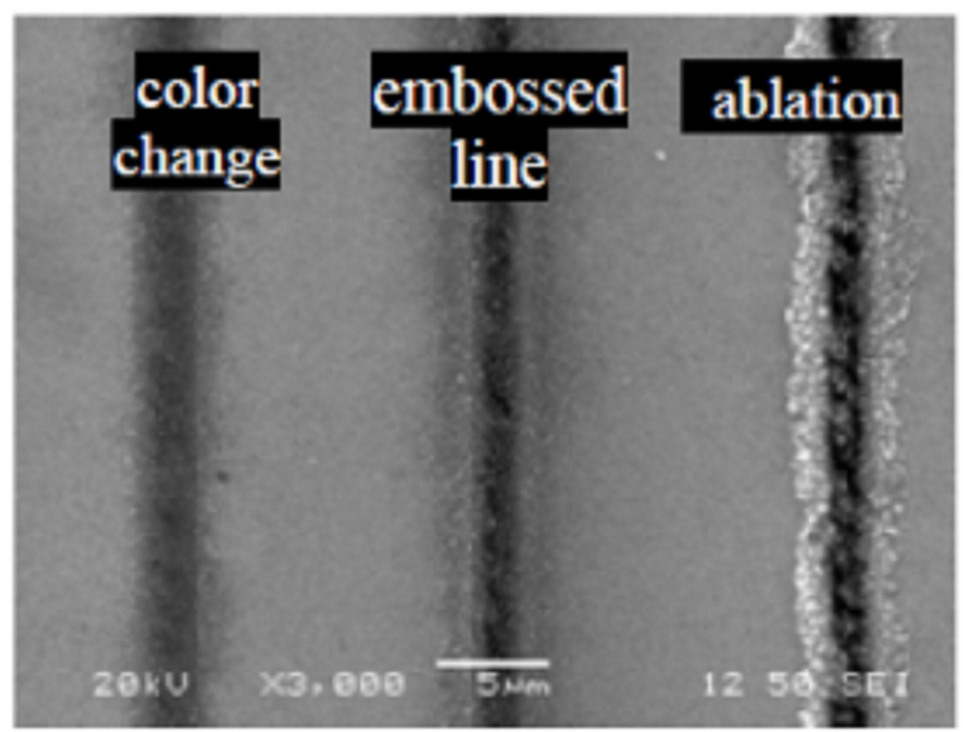

Figure 4.1: SEM image of silicon surface after irradiation with Femtosecond Laser Pulses at 1 W at the various pulse frequency (left to right: $8 \mathrm{MHz}\left(14 \mathrm{~J} / \mathrm{cm}^{2}\right), 13 \mathrm{MHz}\left(9 \mathrm{~J} / \mathrm{cm}^{2}\right)$ and $26 \mathrm{MHz}\left(4.5 \mathrm{~J} / \mathrm{cm}^{2}\right)$

With carefully controlled laser parameters, embossed lines can be created consistently, as shown in Figure 4.2. It is also noticed that this process has much better repeatability at 
$26 \mathrm{MHz}$ than at $13 \mathrm{MHz}$.

Figure 4.3 represents optical microscope topography images of the irradiated areas. Cross sectional optical microscope images show embossed lines around $100 \mathrm{~nm}$ high and $3.5 \mu \mathrm{m}$ width induced with femtosecond laser pulses with a power of $0.35 \mathrm{~W}$ supplied at $26 \mathrm{MHz}$ (Figure $4.3 \mathrm{a})\left(1.65 \mathrm{~J} / \mathrm{cm}^{2}\right)$ and $40 \mathrm{~nm}$ high and $3 \mu \mathrm{m}$ width when the laser power is $1 \mathrm{~W}$ operating at $13 \mathrm{MHz}\left(9 \mathrm{~J} / \mathrm{cm}^{2}\right.$ ). (Figure 4.3b). Increasing pulse frequency results in increased line height.

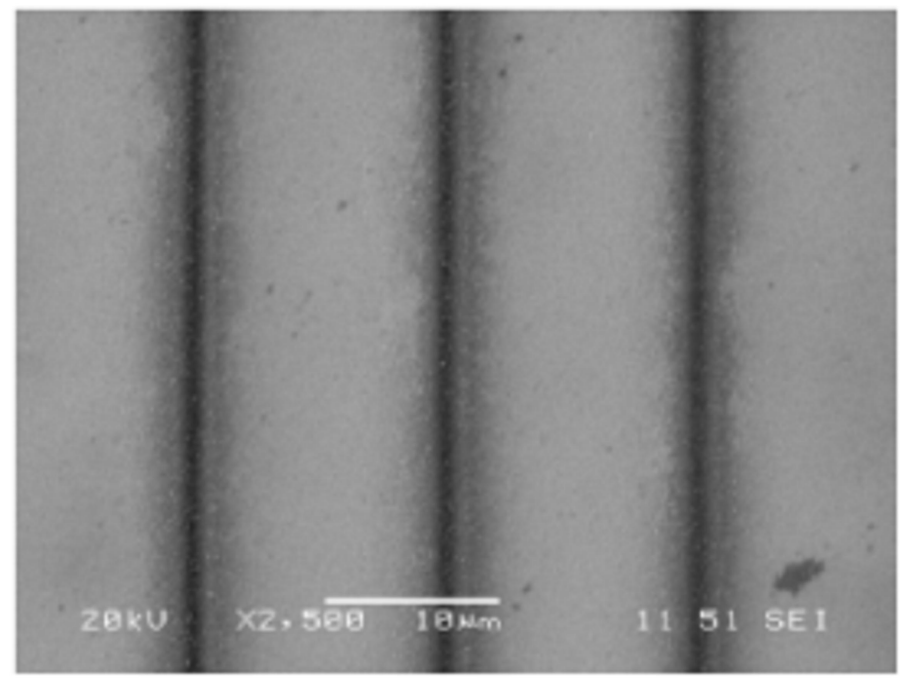

Figure 4.2: SEM image of silicon surface after irradiation with Femtosecond Laser pulses at $0.35 \mathrm{~W}$ at the pulse Frequency of $26 \mathrm{MHz}\left(1.65 \mathrm{~J} / \mathrm{cm}^{2}\right)$.

In Figure 4.4a, the EDX result clearly shows the presence of oxygen in irradiated lines which are believed to be due to Si-O-Si bounds. In an effort to verify the elemental composition of the embossed lines, a micro-Raman spectroscopic analysis was conducted. Back scattering micro-Raman analysis was performed at room temperature using the $532 \mathrm{~nm}$ line of the $\mathrm{Ar}$ ion laser source. The Raman spectroscopic measurements on the processed samples showed 


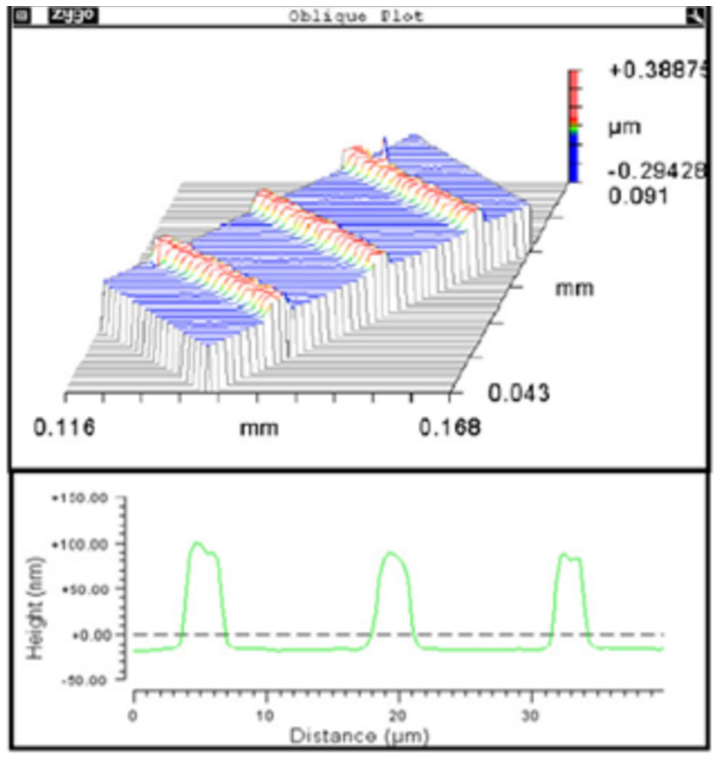

(a)

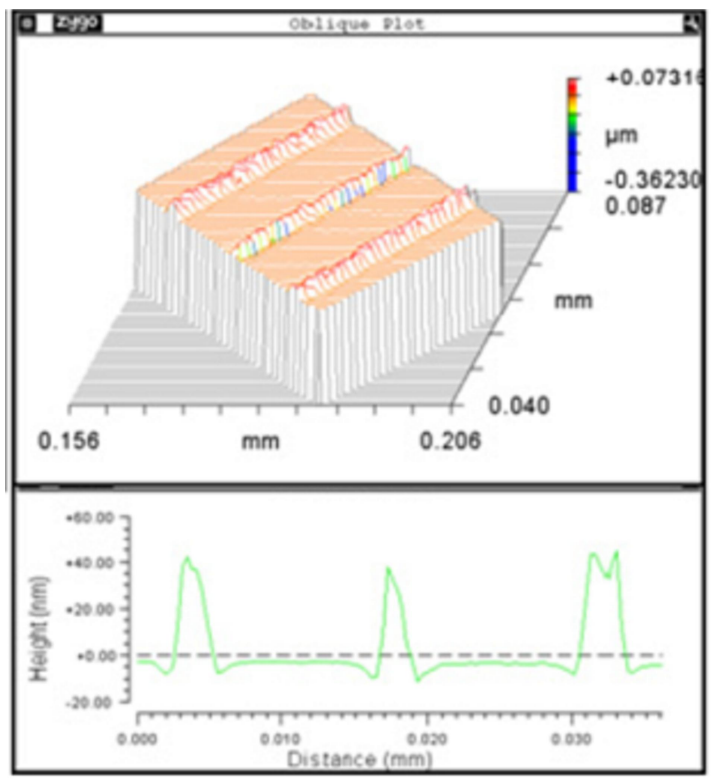

(b)

Figure 4.3: (a) Optical microscope topography and cross sectional image $\left(26 \mathrm{MHz}, 0.35 \mathrm{~W}, 1.65 \mathrm{~J} / \mathrm{cm}^{2}\right)$, (b) optical microscope topography and cross sectional image $\left(13 \mathrm{MHz}, 1 \mathrm{~W}, 1 \mathrm{~J} / \mathrm{cm}^{2}\right)$

a sharp peak at the wavenumber of $519 \mathrm{~cm}^{-1}$, which is the characteristic peak of silicon. Moreover, a hump was observed at the wavenumber of $954 \mathrm{~cm}^{-1}$ indicating the existence of silicon oxide $[51,55]$. (See Figure 4.4b). Therefore, it can be concluded that the irradiated zone was converted into silicon oxide.

\subsection{Numerical Model for Surface Temperature Calcu- lation}

The growth of an oxide layer can be explained by a fundamental thermal model $[37,51]$. Recent simulations using various thermal models have shown that thermal oxidation of 

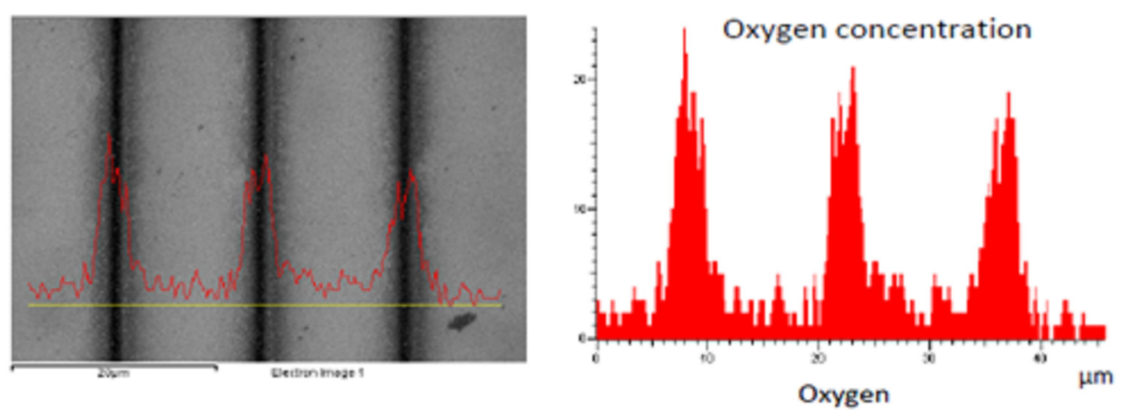

(a)

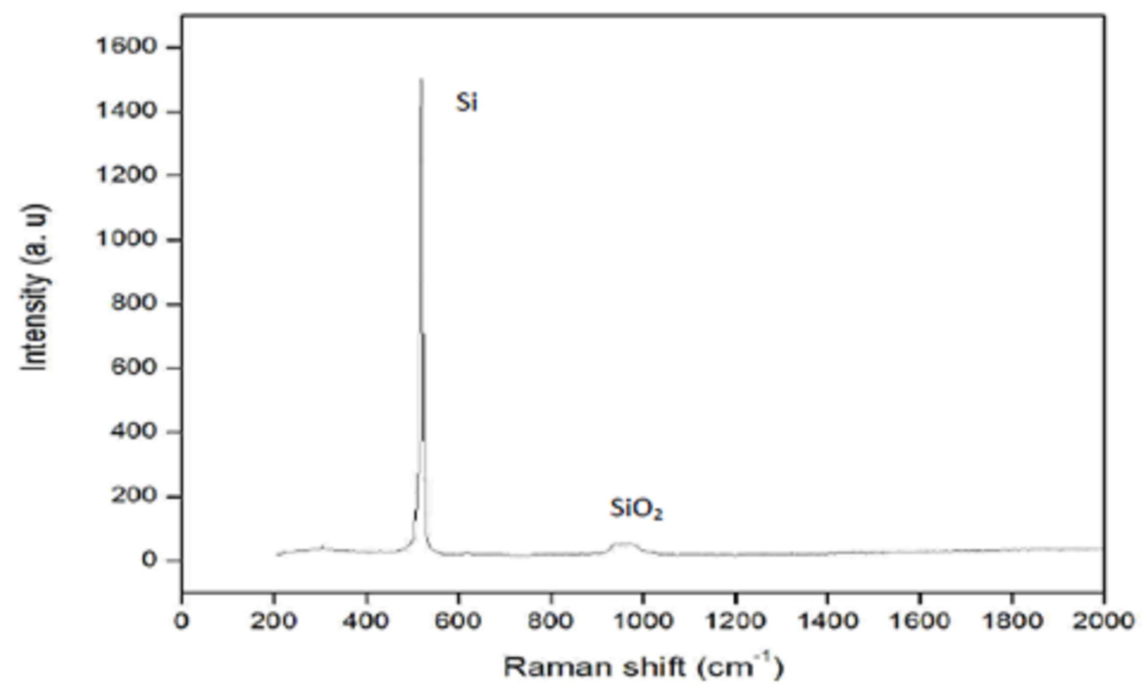

(b)

Figure 4.4: (a) Graph of EDX spectroscopy analysis (b) Graph of Micro-Raman spectroscopy analysis

silicon takes place at temperatures in the range of $700 \sim 1300{ }^{\circ} \mathrm{C}[37,56-59]$. In the following analysis, it is assumed that if the temperature of the silicon surface is brought up and kept in the range of $700 \sim 1300{ }^{\circ} \mathrm{C}(1000 \sim 1600 K)$, oxidization occurs.

In the analyses of the heat accumulation, the surface temperature at the end of a laser pulse, $\Delta T$, can be approximately calculated by the following formula [25]. 


$$
\Delta T=\frac{I_{a} r^{2} \tau_{1}}{4 \sqrt{\pi} k t \sqrt{D t}}
$$

where $I_{a}$ is the absorbed laser light intensity, $r$ is the radius of the focal spot, $\tau_{1}$ is the pulse duration, $k$ is thermal conductivity, $D$ is thermal diffusivity of the silicon and $t$ is the pulse interval. Pulse interval $t$ is given by $t=1 / f$ ( $f$ is the pulse frequency).

The accumulated temperature rise at the end of $N^{t h}$ pulse is $T[51,60]$ :

$$
T=N \times \Delta T=N \frac{I_{a} r^{2} \tau_{1}}{4 \sqrt{\pi} k t \sqrt{D t}}
$$

The light intensity, $I_{a}$, can be estimated by the reflection coefficient, $R$, at a wavelength of $520 \mathrm{~nm}$ and residual energy coefficient, $K[51,61]$ :

$$
I_{a}=K(1-R) I_{i}
$$

The incident light intensity, $I_{i}$, is calculated from laser power, $P$, pulse frequency, $f$, which is the reciprocal of pulse interval, $t$, and laser spot diameter, $d$.

$$
I_{i}=\frac{4 P}{\tau_{1} \pi d^{2} f}
$$

Substituting Equation 4.3 and Equation 4.4 in Equation 4.2, yields:

$$
T=\frac{N \times K(1-R) P}{4 K \sqrt{\pi^{3} D t}}
$$


Previous studies showed that the reflection coefficient of silicon during irradiation with the femtosecond laser is 0.393 at a wavelength of 488 and 0.329 at the wavelength of 800 $\mathrm{nm}[51,62]$.

Thus, the reflection coefficient of silicon at a wavelength of $520 \mathrm{~nm}$ is calculated to be around 0.38 . When the fluence is less than ablation threshold, experiments revealed that $K$ is around 0.8 for silicon [63]. In this experiment, the speed of scanning is $500 \mathrm{~mm} / \mathrm{s}$, the thermal conductivity $(k)$ is $155 \mathrm{~W} /(\mathrm{mK})$ and the thermal diffusivity is $8.5 \times 10^{-5} \mathrm{~m}^{2} / \mathrm{s}$. [64]. Figure $4.5 \mathrm{a}$ shows the surface temperature at various pulse frequency and average power calculated from Equation 4.4.

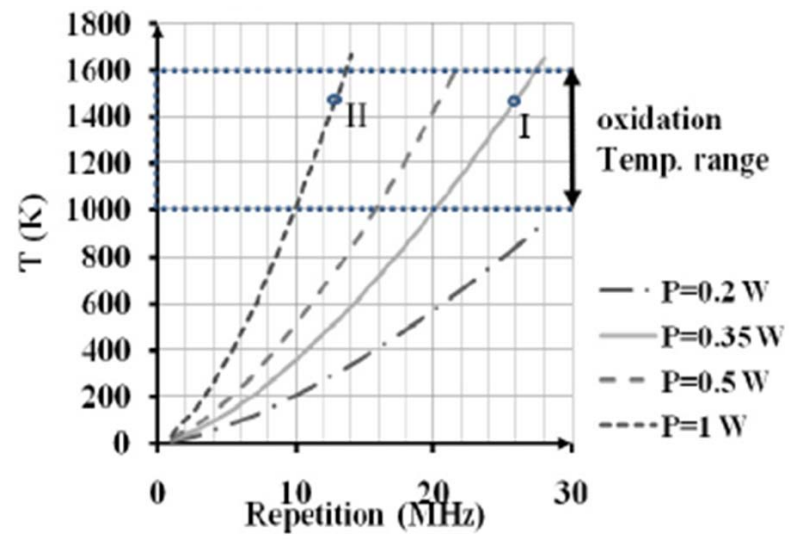

(a)

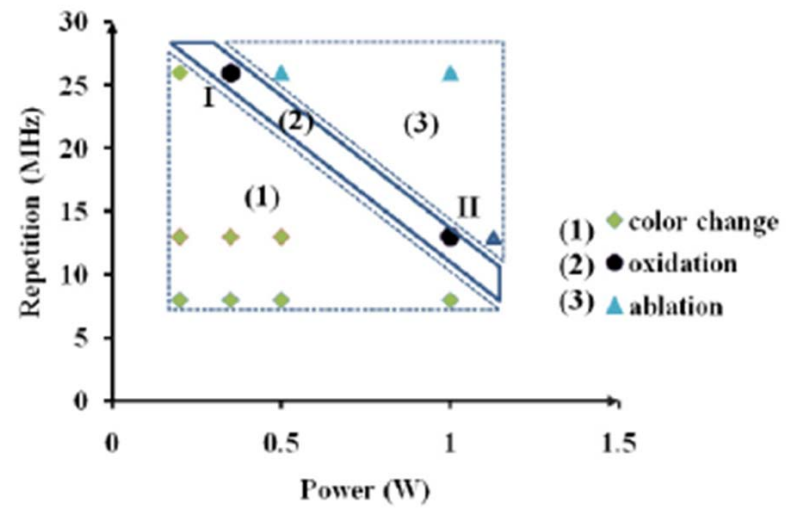

(b)

Figure 4.5: (a) theoretical results based on Equation 4.4 (Relation between $\mathrm{T}$ and frequency at various powers) and (b)Experimental results

As shown in Figure 4.5a, the temperature of the silicon substrate can be brought up to $1450 \mathrm{~K}$ using 214 femtosecond pulsed laser light at $0.35 \mathrm{~W}$ with the frequency of $26 \mathrm{M} \mathrm{Hz}$ (point I) and at $1 \mathrm{~W}$ with the frequency of $13 \mathrm{MHz}$ (point II), which could lead to the formation of silicon oxide on the substrate surface. This calculation is in agreement with 
the results of the experiment. Figure 4.5b maps the range of laser parameters used for the experiments and the observed morphology alteration. Only two points, (I) and (II), fall into the oxidation temperature ranges in Figure 4.5a, and this is where the embossed lines were observed. Figure 4.5b defines three zones (1), (2) and (3) which can be named as the color-change, oxidation and ablation zone, respectively. As Figure 4.5b presents, oxidation occurs only at a narrow band of Zone (2), which is slightly below the border line of ablation. This means that direct oxidation with the femtosecond laser requires a stringent parameter control. Even a slight deviation in laser parameters, for examples fluctuation in laser power, will result in surface temperature drift and will not result in oxidation. Therefore, higher pulse frequency is preferred for oxidation since at the same laser power the pulse energy fluctuation is lower at higher pulse frequency. Furthermore, as shown in Figure 4.5b, the tolerance of the power for the oxidation process could be around $\pm 0.1 \mathrm{~W}$ and the percentage of power stability is constant (1percent), thus the absolute power fluctuation is less in lower powers such as $0.35 \mathrm{~W}$ rather than $1 \mathrm{~W}$ and higher. This explains why better repeatability was observed at $26 \mathrm{MHz}$ during the experiments [51].

Increasing pulse frequency has a noticeable influence on the heat accumulation process; high pulse frequency results in both decreasing the heat dissipation between pulse intervals and increasing surface temperature at the end of $N$ th pulse. Therefore, with the same laser power, the surface temperature would be higher at $26 \mathrm{M} \mathrm{Hz}$ in comparison with $13 \mathrm{M} \mathrm{Hz}$ [30]. Consequently, increasing pulse frequency results in increasing the line height.

Using high repetition $(\mathrm{MHz})$ ultra fast (fs) laser pulses enable us to bring the temperature of the target surface to the oxidation temperature range and keep it in this range. In other words, the laser fluence can be controlled to be below the ablation threshold and in the 
oxidation range by using the megahertz frequency femtosecond laser irradiation [54].

\subsection{Summary}

In this study, a new method was proposed for laser direct patterning of silicon oxide on Si-substrate, using a megahertz femtosecond pulsed laser. Embossed lines of silicon oxide around $3 \sim 4 \mu \mathrm{m}$ width and less than $100 \mathrm{~nm}$ height were generated by irradiation with laser at $1 \mathrm{~W}\left(9 \mathrm{~J} / \mathrm{cm}^{2}\right)$, at $13 \mathrm{MHz}$ and $0.35 \mathrm{~W}\left(1.65 \mathrm{~J} / \mathrm{cm}^{2}\right)$, at 26 mega hertz scanning speed: $500 \mathrm{~mm} / \mathrm{s}$ ). It can be concluded that the oscillator laser systems are the suitable ones for the laser oxidation process, since they provide laser pulses with the low power, wide range of frequency and better laser power stability. An analytical model was developed for the calculation of the average surface temperature after $n$-pulses; it was found that for a constant power and a constant repetition rate at megahertz, an increase in the pulse number does not correspond to a significant increase in the surface temperature. Moreover, at the controlled laser power level, the surface temperature will not exceed the melting point of silicon. A SEM, a Micro-Raman and EDX spectroscope and an optical microscope were employed to characterize the oxide layers. Using the high frequency femtosecond pulsed laser makes this technique particularly suitable for rapid prototyping and customscale manufacturing for a wide variety of applications in MEMS, NEMS, fabrication of semiconductor and LOC systems which demand for flexibility in re-design. This technique does not require a photomask and any redesign can be easily done in the large scanning fields

$(m m \times m m)$ by using Computer-Aided Design (CAD) software for the beam scanning [51,54]. 


\section{Chapter 5}

\section{Direct-writing of Amorphorized}

\section{Silicon Thin-film on Si Substrate}

His chapter presents development of an alternative maskless nanolithography by
a combination of femtosecond laser amorphization of silicon and wet etching.
Using a high repetition femtosecond laser $(\mathrm{MHz})$ makes it possible to control laser fluence below ablation threshold which causes the crystalline silicon phase transition to an amorphous state. The induced thin layer of amorphous silicon is then used as an etch stop in the following wet chemical etching $(\mathrm{KOH})$ for maskless lithography. This may lead to a promising solution for maskless nanolithography because the laser beam is a non-contact probe and can be scanned at very high speed. 


\section{$5.1 \quad$ Procedure}

Figure 5.1 illustrates the proposed maskless fabrication technique, which combines femtosecond laser irradiation and wet chemical etching. First, silicon wafers are irradiated with femtosecond pulses with pulse energy well below the damage threshold. Then, the treated samples are etched in a $\mathrm{KOH}$ solution [15].

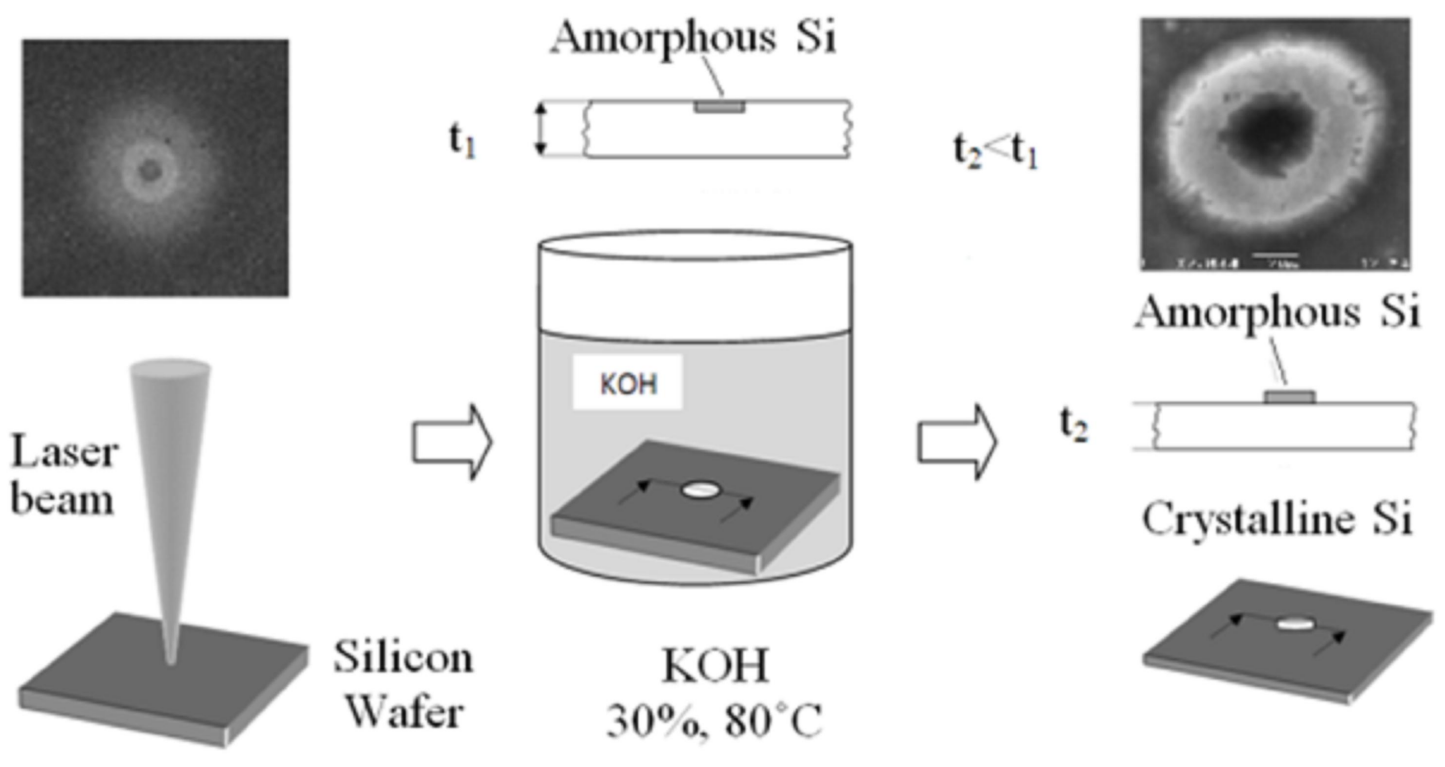

Figure 5.1: Process of experiment

To study the silicon amorphization, undoped $<100>$ oriented silicon wafers were used. Prior to laser irradiation, the samples were cleaned with alcohol and ultra pure water. The theoretical spot size on the samples is calculated to be $10.38 \mu \mathrm{m}$ in diameter. During the experiment the spot size may be bigger due to scatter and misalignment. The average laser fluence was calculated to be $0.15 \sim 0.35 \mathrm{~J} / \mathrm{cm}^{2}$ at the repetition rate of 13 and $26 \mathrm{MHz}$. In the present experiment the dwell time is $0.1 \mathrm{~ms}$ and scanning speed of line features is in the 
range of $100-150 \mathrm{~mm} / \mathrm{s}$. Etched samples were examined under a SEM, a Micro-Raman and EDX spectroscope to evaluate the quality of amorphous layer and the etching process. Back scattering micro-Raman analysis was performed at room temperature using the $532 \mathrm{~nm}$ line of the Ar ion laser source [15].

\subsection{Results and Discussion}

Figure 5.2b shows morphology after irradiation with sub-threshold femtosecond laser pulses. Surface morphologies vary according to the intensity distribution of a Gaussian beam. At the center region where the laser fluence was the highest, ablation occurred, as expected. In this zone the intensity of a Gaussian beam is above the surface damage threshold. Therefore, material removal takes place. The next annulus could be a thin layer of amorphous or polycrystalline silicon because when observed under SEM this region showed a difference in reflectivity. The amorphous phase could be formed on crystalline silicon because of the rapid cooling after the femtosecond excitement, which is up to $10^{13}-10^{15}{ }^{\circ} \mathrm{C} / \mathrm{s}$ [65]. Therefore, there is not enough time for the material to return to the crystalline phase. It is noteworthy that the phase change on crystalline silicon was restricted only within a certain range of laser fluence $[65,66]$. Above this fluence range, for example, at the center of the Gaussian beam, high pulse energy causes ablation. It was observed that after etching the center zone was removed by etchant. Thus, the central zone was not in the amorphous state [15].

In the outermost annulus, it was not expected to see any phase change because the laser energy fluence was lower than the amorphous modification threshold. However, the SEM image (Figure 5.2b) shows that outside the unmodified annulus, there was another modified 


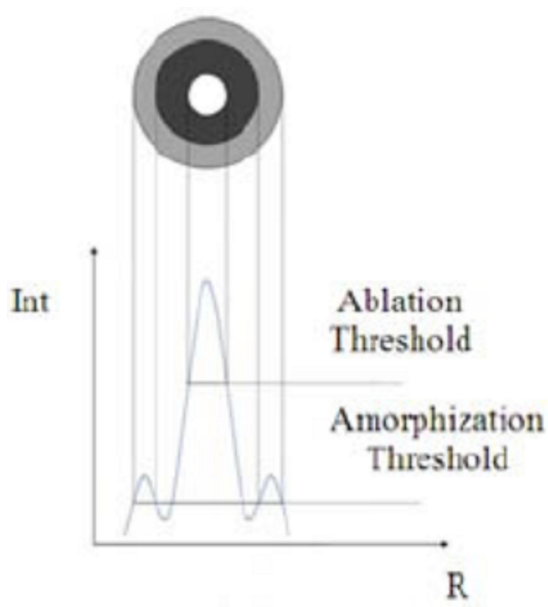

(a)

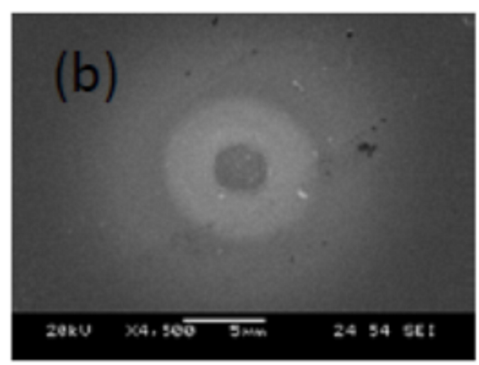

(b)

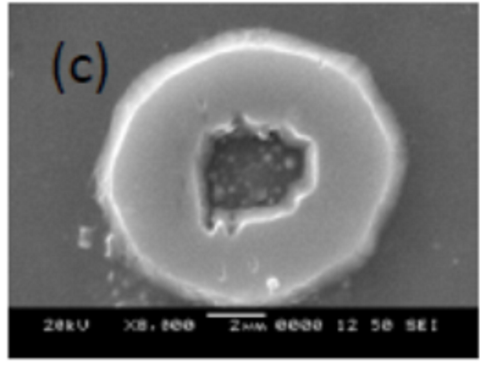

(c)

Figure 5.2: (a) Schematic drawing of laser intensity and surface modification on silicon substrate. (b) Silicon wafer after irradiation with sub-threshold. (c) Silicon wafer after etching

annulus. This annulus can be attributed to the secondary intensity peak generated by diffraction. After etching, this region was not seen in the SEM images. Laser energy fluence at the secondary peak was not high enough to completely change the phase of the material. It was only sufficient to slightly modify the material; therefore, it was removed in the postirradiation etching process. After etching, it was observed that the non-irradiated area and the central region of the laser irradiation were etched by $\mathrm{KOH}$; whereas, the annulus around the central region of the laser spot was scarcely etched. Consequently, a ring feature stood out on the etched silicon substrate, suggesting that the amorphous layer was resistant to $\mathrm{KOH}$. The internal quadrilateral shapes were due to the orientation of the silicon crystal (see Figure 5.3). This observation is in agreement with the etch rate of amorphous silicon reported by Kawasegi N. et al. [27, 29]. According to Kawasegi et al, the etch rate of amorphous silicon which formed by magnetron spattering was over 30 times lower than that of crystalline silicon $<100>$. 


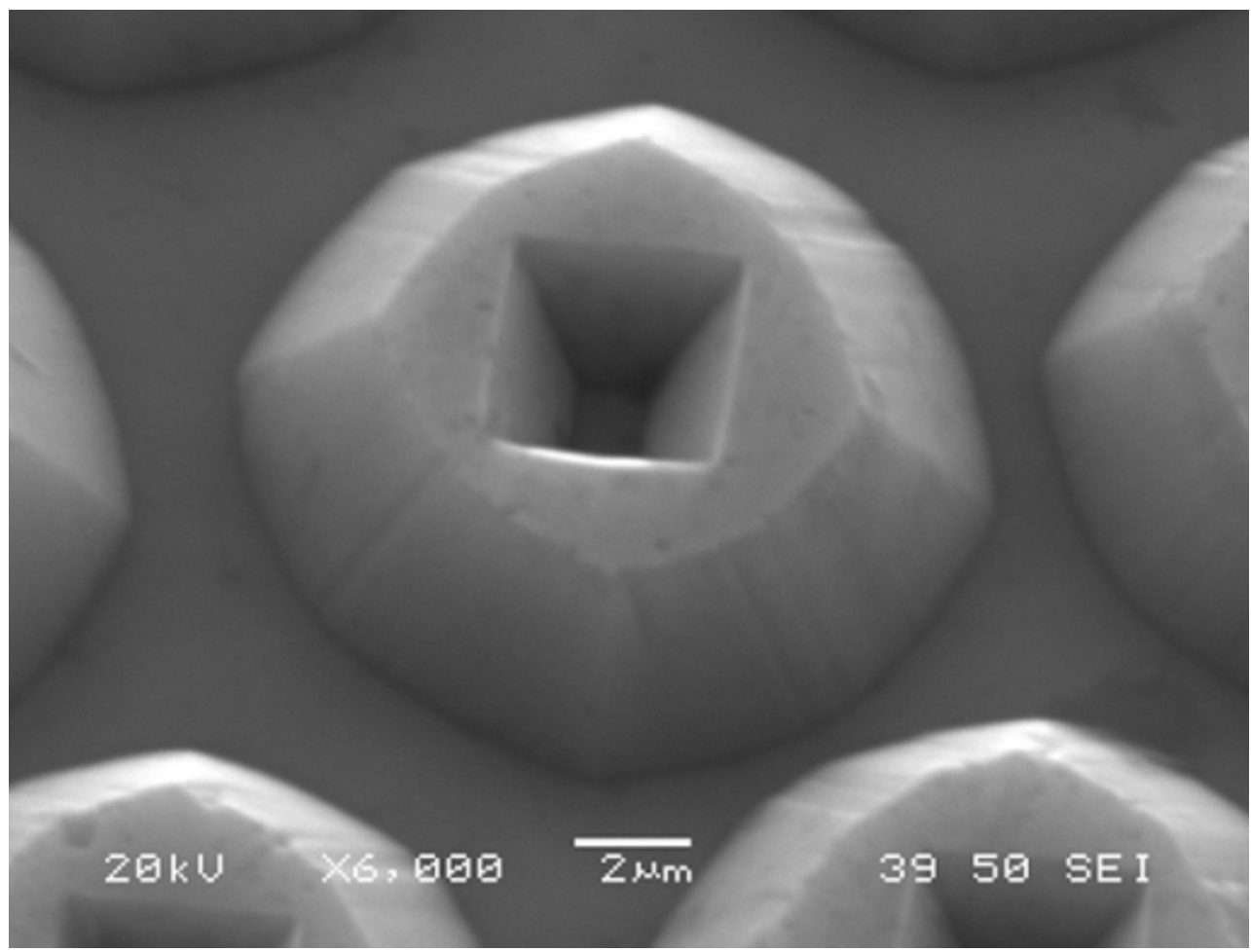

Figure 5.3: SEM image of ring feature after etching in $\mathrm{KOH}$ solution for $15 \mathrm{~min}$ at $45{ }^{\circ} \mathrm{C}$ (Average laser fluence: $\left.0.27 \mathrm{~J} / \mathrm{cm}^{2}\right)$

A well defined circular dot instead of a ring feature is more applicable in most applications. In order to get a circular dot after wet etching, the intensity of the laser beam should be controlled such that the peak intensity does not exceed the ablation threshold. Figure 5.4 gives SEM images of features obtained after wet etching. The round pad was obtained by reducing the pulse energy, as shown in Figure 5.4(b). The pad was around $2 \mu m$ in diameter, which is far smaller than the estimated focused laser spot. This demonstrated that the intensity of the laser beam can be accurately controlled such that only a small section surrounding the central peak of the Gaussian beam is above the amorphization threshold, enabling the creation of features smaller than the focused spot. Using the same 
technique, sub-100 $\mathrm{nm}$ ablated features have been created with a laser spot of $2 \mu \mathrm{m}$ diameter [38].
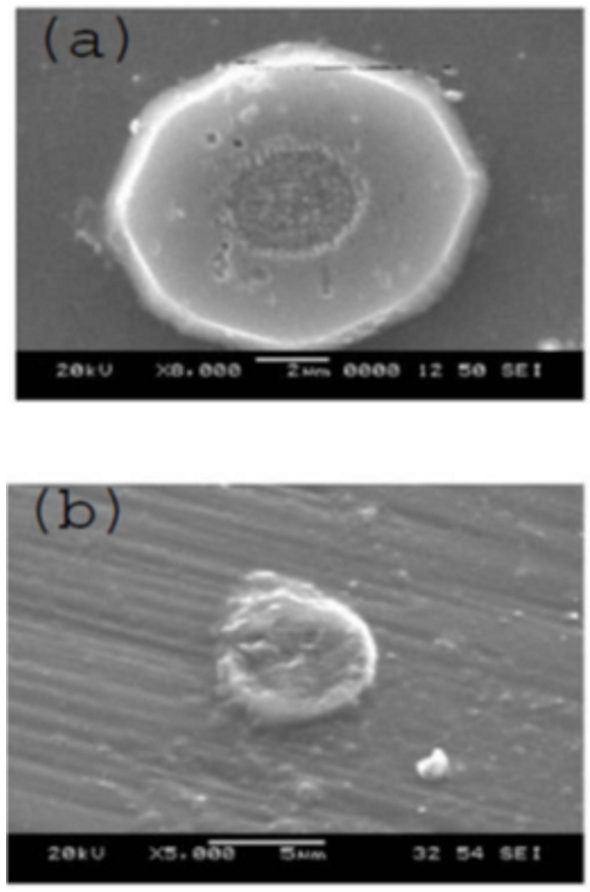
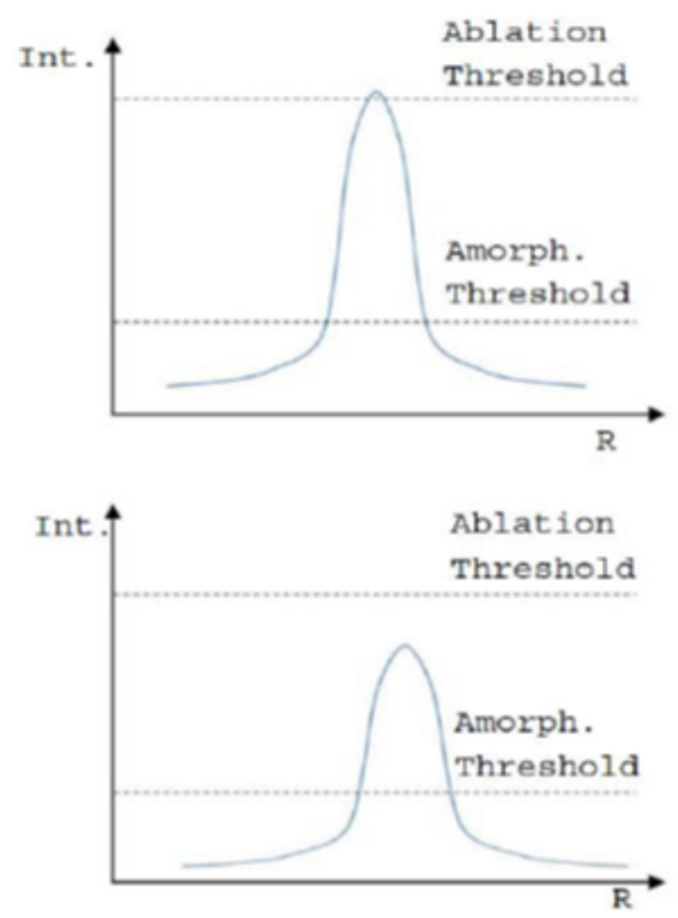

Figure 5.4: SEM images features after 3 min etching) (a) ring feature at average laser fluence of $0.3 \mathrm{~J} / \mathrm{cm}^{2}$ (b) solid round at $0.2 \mathrm{~J} / \mathrm{cm}^{2}$

The fabrication of micro and nanometer-scale lines may be useful for such diverse applications as micro and nano scale length standards, optical grating fabrication, or in the development of novel sensors [67]. Figure 5.5 presents line features generated with the described maskless lithography. The thickness and width of the induced layer (amorphous layer) were calculated to be approximately $1.4 \mu \mathrm{m}$ and $6 \mu \mathrm{m}$. The average laser fluence was $0.28 \mathrm{~J} / \mathrm{cm}^{2}$ 
at the repetition rate of $26 \mathrm{MHz}$. As expected, the part of the substrate that was not irradiated with the laser beam was dissolved after etching and a pyramid-like structure with sidewall slope at $54.74^{\circ}$ was formed (See Figure 5.5, zone (b)), while, the irradiated zone was not etched in the etchant solution and the angle of the sidewall of the irradiated zone was $90^{\circ}$ (See Figure 5.5, zone (a)). It can be concluded from this observation that the irradiated zone is not in crystalline state. Further analyses performed by EDX and Micro-Raman demonstrated that the irradiated zone is silicon in the amorphous state rather than the crystalline state or silicon oxide [15].

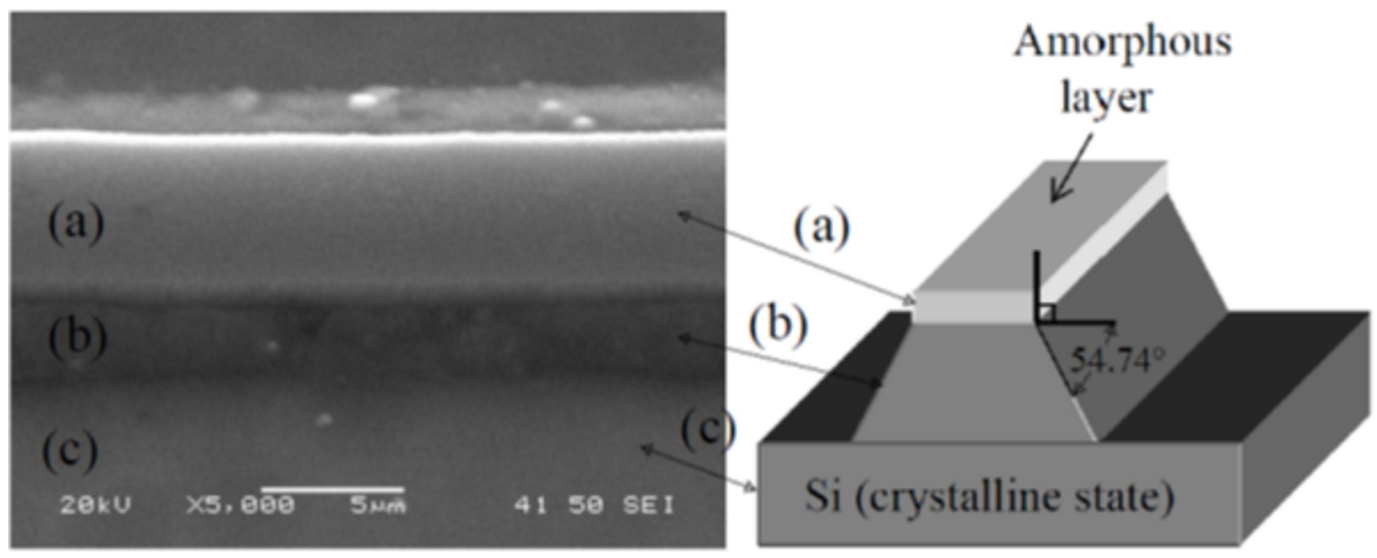

Figure 5.5: SEM image (at the incident angle of $35^{\circ}$ ) of line feature after etching in $\mathrm{KOH}$ solution (Pulse energy: $0.28 \mathrm{~J} / \mathrm{cm}^{2}$ )

Figure 5.6 presents line features generated with a scanning laser spot of various pulse energies. As pulse energy reduces, the line width reduces. The smallest line width achieved with the $10 \mu \mathrm{m}$ laser spot was around $150 \mathrm{~nm}$, as shown in Figure 5.6(d). The line width is around $1 / 67$ of the laser spot diameter. Since the laser beam can easily be focused down to $1-2 \mu m$ with a focusing lens of a high numerical aperture, together with the adoption of laser frequency doubling or tripling, nano-scale feature size can be expected [15]. 

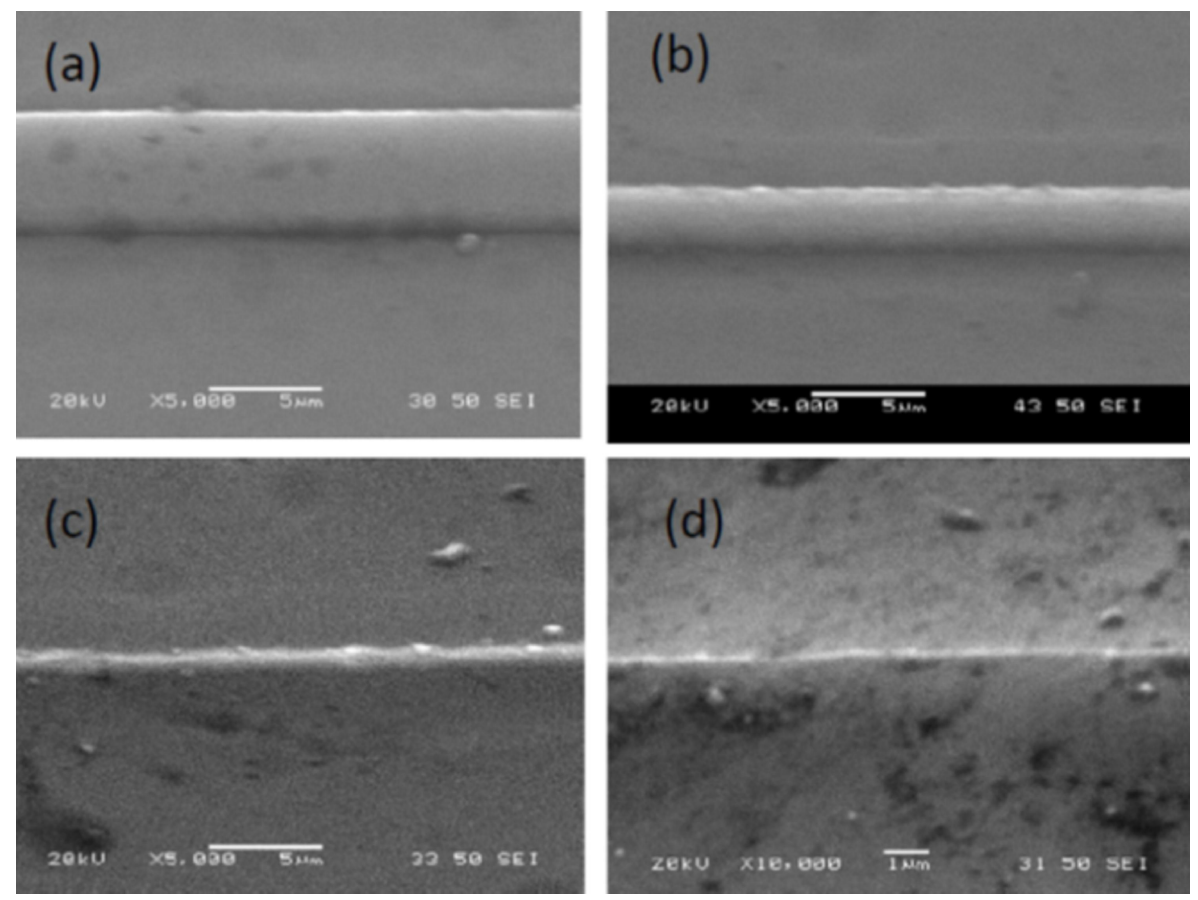

Figure 5.6: (a) $5 \mu \mathrm{m}$ linewidth at pulse energy of $0.28 \mathrm{~J} / \mathrm{cm}^{2}$ (at $26 \mathrm{MHz}$ ) (a) $2.5 \mu \mathrm{m}$ linewidth at pulse energy of $0.24 \mathrm{~J} / \mathrm{cm}^{2}$ (at $26 \mathrm{MHz}$ ) (b) $1.0 \mu \mathrm{m}$ linewidth at pulse energy of $0.2 \mathrm{~J} / \mathrm{cm}^{2}$ (at $26 \mathrm{MHz}$ ) (c) $150 \mathrm{~nm}$ linewidth at pulse energy of $0.08 \mathrm{~J} / \mathrm{cm}^{2}$ (at $26 \mathrm{MHz}$ )

Investigations done previously indicated that upon the sub-damage threshold laser irradiation on silicon wafers, a thin layer on the top surface has been converted to a chemical compound, such as silicon oxide and silicon hydroxide [35,68]. However, this is not the case in this experiment. EDX analysis of the irradiated zone excluded the possibility of compound formation since no trace of oxygen was observed (Figure 5.7).

Raman Spectroscopy measurements were performed to study the crystal properties of different zones observed after laser irradiation. Raman spectrum is dominated by the signal at $517 \mathrm{~cm}^{-1}$ which corresponds to crystalline silicon. In contrast the broad band observed around $506.9 \mathrm{~cm}^{-1}$ is characteristic for amorphous silicon [62]. Therefore, it can be con- 

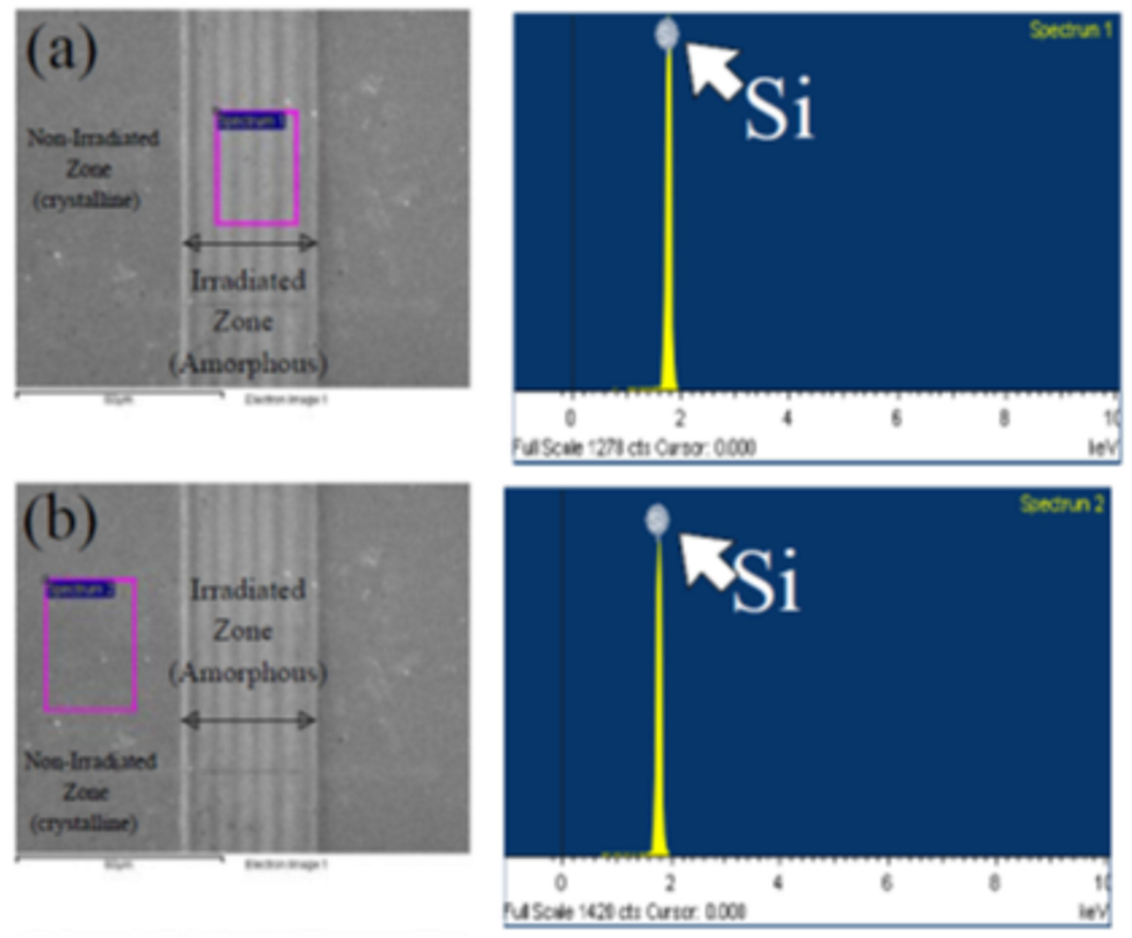

Figure 5.7: EDX analysis of (a) irradiated zone and (b) non-irradiated zone

cluded that the induced zone is amorphous silicon rather than silicon oxide (Figure 5.8).

Compared with approaches that are based on AFM, the described method has many advantages. Initially, at $3000 \mathrm{~mm} / \mathrm{sec}$ probe scanning speed the processing time of patterning is next to zero for a feature with dimensions within the scan field. On the other hand, it takes a few minutes to complete the patterning if an AFM is used [28, 29]. Secondly, the scanning filed of a laser system is much larger than that of an AFM: $1 \mathrm{~mm} \times 1 \mathrm{~mm}$ is normally associated with a $10 \mu \mathrm{m}$ laser spot. Therefore, sub-millimeter devices can be fabricated without stitching and, for large scale patterning, cell stitching can be performed with more tolerance on misalignment between cells. Finally, due to the non-contact nature of the laser probe, this process does not suffer issues that are normally associated with tool 
wear.

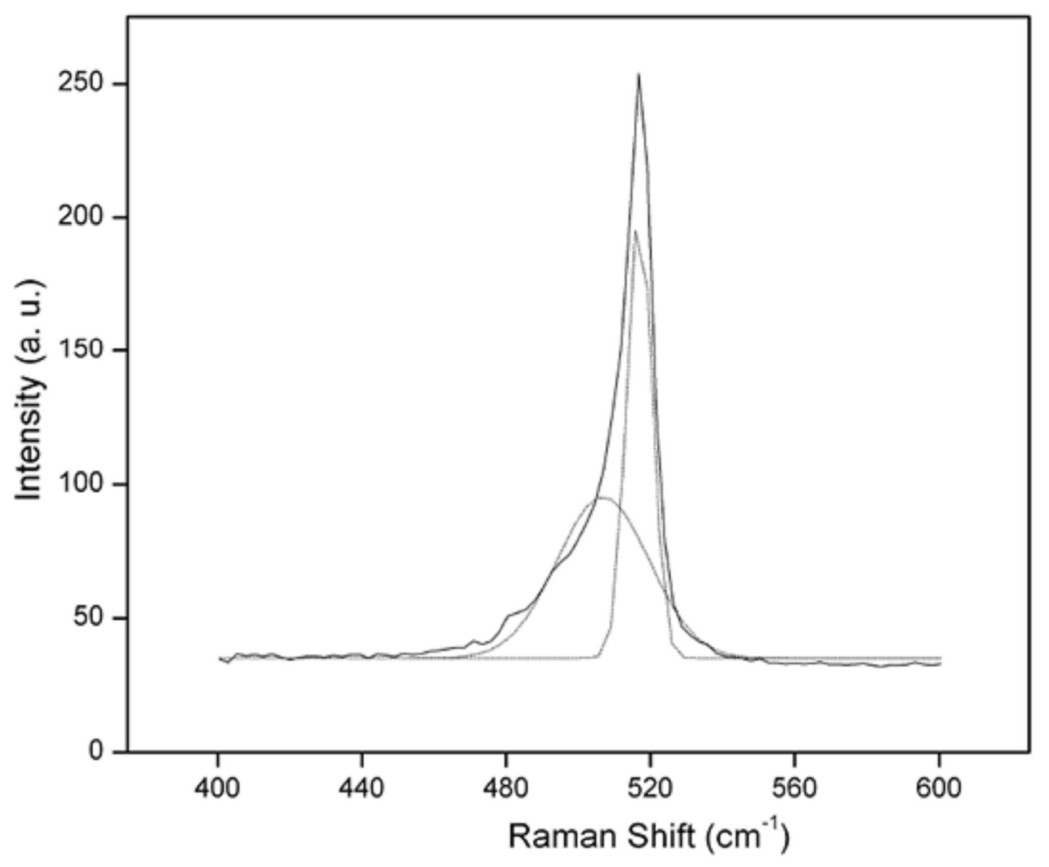

Figure 5.8: Raman spectroscopy graph

\subsection{Summary}

Amorphous layers formed by laser irradiation acted as an etch stop in $\mathrm{KOH}$, and micro and nano-features were fabricated by this method $\left(0.15 \sim 0.35 \mathrm{~J} / \mathrm{cm}^{2}\right.$ at 13 and $26 \mathrm{MHz}$, dwell time: $0.1 \mathrm{~ms}$, scanning speed: $100-150 \mathrm{~mm} / \mathrm{s}$ ). The Micro-Raman and EDX spectroscopy analysis revealed the amorphous state of the modified silicon. An important advantage of this technique is the use of femtosecond laser pulses which make it particularly suitable for rapid prototyping and custom-scale manufacturing for a wide variety of applications in MEMS, NEMS, fabrication of semiconductors and LOC systems. The features smaller 
than $1 / 67$ of the focused spot size were generated by controlling the laser fluence and etching parameters. Submicron and nano-scale feature size can be expected by reducing the focused spot size and optimizing etching parameters. Moreover, the wet etch process guarantees well defined shape and smooth finishing in the final products. 


\section{Chapter 6}

\section{Effect of Laser Parameters}

\footnotetext{
T $\mathrm{N}$, this chapter, a theoretical and experimental analysis of the effect of the laser parameters is carried out. These include repetition rate, pulse width and number of pulses

L on the quality of amorphorized area and the size of the feature fabricated by high repetition (MHz) femtosecond laser irradiation.
}

\subsection{Laser Parameters and Laser Annealing of Silicon}

As shown in Table 6.1, the oxidation process was conducted with lower laser powers and higher scanning speeds $\left(0.35\right.$ and $\left.1 \mathrm{~W}, 500 \mathrm{~mm} / \mathrm{s}^{2}\right)$ in comparison with the amorphization process $\left(4.5-6.5 \mathrm{~W}, 100-150 \mathrm{~mm} / \mathrm{s}^{2}\right)$. In the amorphization process, in order to obtain a thin layer of amorphorized silicon on top of the silicon substrate, the surface target of silicon should melt and resolidify (Chapter 5); thus, the scanning speed (number of pulses) should 
be high enough to melt the substrate. However, in the oxidation process, melting the target surface should be avoided (higher scanning speed up to $500 \mathrm{~mm} / \mathrm{cm}^{2}$ ).

\begin{tabular}{|c|c|c|}
\cline { 2 - 3 } \multicolumn{1}{c|}{} & Oxidation & Amorphization \\
\hline Wavelength $(\mathrm{nm})$ & 515 & 1030 \\
\hline Spot diameter $(\mu \mathrm{m})$ & 1.02 & 10.4 \\
\hline Laser power $(\mathrm{W})$ & $0.35,1.0$ & $4.5-6.5$ \\
\hline Laser fluence $\left(\mathrm{J} / \mathrm{cm}^{2}\right)$ & $1.65,9$ & $0.7-0.3$ \\
\hline Frequency $(\mathrm{MHz})$ & 13,26 & $8-13$ \\
\hline Pulse width $(\mathrm{fs})$ & 214 & $214-3500$ \\
\hline Scanning speed $(\mathrm{mm} / \mathrm{s})$ & 500 & $100-150$ \\
\hline
\end{tabular}

Table 6.1: Laser parameters for the oxidation and amorphization process

As Chapter 4 presents, oxidation occurs only at a narrow band below the border line of ablation. This means that direct oxidation with laser pulses requires a stringent parameter control. Even a slight deviation in laser parameters, for examples fluctuation in laser power, will result in surface temperature drift and will not result in oxidation. Also, in the laser system used for this research, parameters such as pulse duration and frequency cannot be varied continuously and only discrete variations are possible to be chosen by users. These limitations explain why the oxidized areas were only evident at two points (Chapter 4). Due to these limitations, the effect of laser parameters on laser annealing of silicon were investigated only for the amorphization process, which is believed to have resemblance to the oxidation process. Thus, the results of the effect of laser parameters in the amorphization process presented in this chapter, can be used for the oxidation process as well. 


\subsection{Experimental Setup}

To study the silicon amorphization, undoped $<100>$ oriented silicon wafer were used. First, silicon samples were rinsed in acetone and then in pure water to remove contamination and debris. After that, samples were irradiated with femtosecond pulses with pulse energy well below the damage threshold to create predetermined patterns of amorphous silicon. Finally, the treated samples were etched in a wet alkaline etchant $(\mathrm{KOH})[15,41]$.

The focused laser spot diameter was calculated to be around $10.4 \mu \mathrm{m}$ in diameter at the wavelength of $1030 \mathrm{~nm}$. In the experiment, the pulse repetition rate varied from 8 to $26 \mathrm{MHz}$ and scanning speed is set in the range of $50-150 \mathrm{~mm} / \mathrm{s}$ using $E z C A D^{\odot}$ software. The laser power was measured to be $5.5-7.5 \mathrm{~W}$, which was obtained with the power-meter prior to the beam entering galvo-scanner [41].

Etched samples were examined under a SEM to evaluate the quality of the amorphous layer and the etching process. The results are presented and discussed in the following sections.

\subsection{Results and Discussion}

The laser amorphization/oxidation process of silicon is very complex and highly dependent on laser parameters such as pulse duration, repetition rate and pulse number. 


\subsubsection{Effect of pulse repetition}

Figure 6.1 shows the amorphorized layers obtained at a repetition rate of $8 \mathrm{MHz}$ (average power of $4.8 \mathrm{~W}$ ), $13 \mathrm{M} \mathrm{Hz}$ (average power of $5.6 \mathrm{~W}$ ) and $26 \mathrm{M} \mathrm{Hz}$ (average power of $6.25 \mathrm{~W}$ ), respectively. It is evident that the quality of the amorphorized area is better with the higher repetition rate at $26 \mathrm{MHz}$ and no damage at $26 \mathrm{MHz}$ is visible, even though the average laser power is the highest at $26 \mathrm{MHz}$.
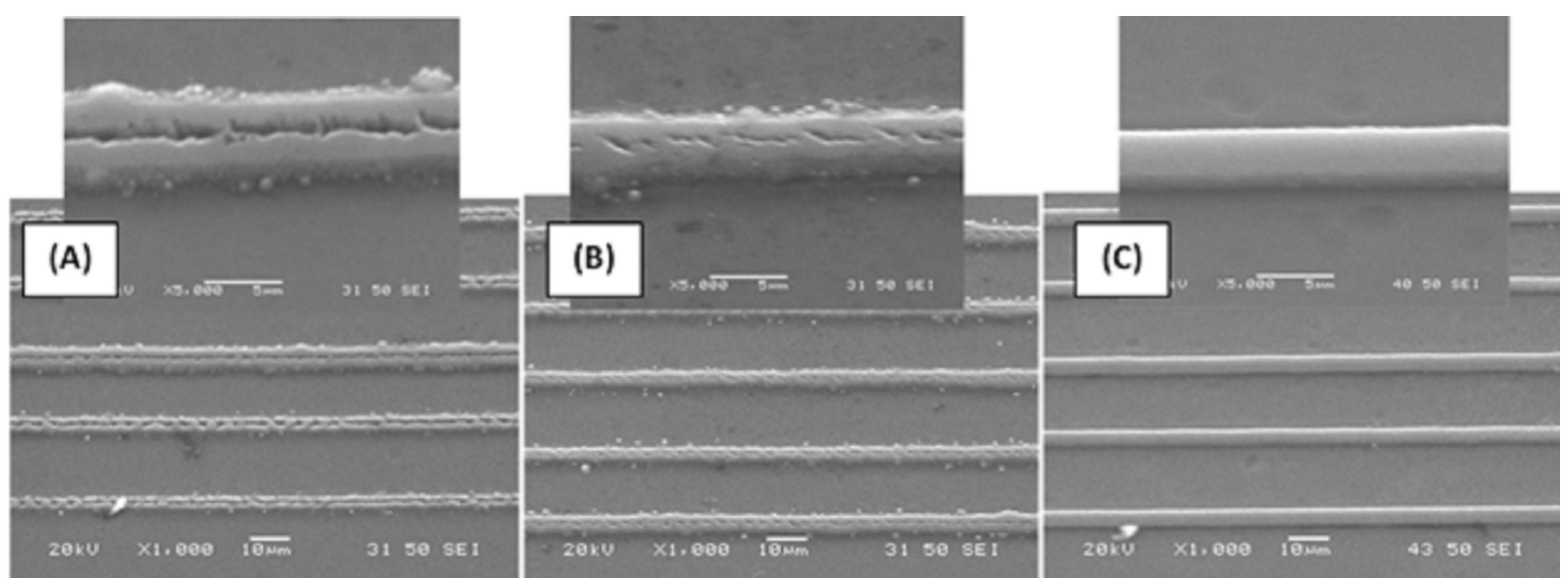

Figure 6.1: SEM images at different pulse repetition rates (pulse duration: 214 fs), (a) 8 MHz, 4.8 W, (b) $13 \mathrm{MHz}, 5.6 \mathrm{~W}$, and (c) $26 \mathrm{MHz}, 6.25 \mathrm{~W}$

Pulse energy, $E_{a}$, is calculated from $E_{a}=\frac{P}{f}$, where $P$ is an average laser power and $f$ is repetition rate. The average fluence per pulse can be calculated from $I_{0}=\frac{E_{a}}{\pi r^{2}}$; where $r$ is a radius of focal point and $E_{a}$ is average pulse energy. Thus, $I_{0}=\frac{P}{f \pi r^{2}}$. As seen in Table 6.2, at lower repetition rate, much higher pulse energy is delivered and therefore a stronger source of thermal diffusion is provided. Moreover, the silicon surface absorption increases with pulse energy as a result of increasing photoionization rate $[69,70]$. With lower repetition rate, fluctuations in pulse energy is also higher and even a slight deviation will result in 
pulse energy overcoming the ablation threshold and causing material breakdown [51,71]. Thus, at the lower repetition rate, the combination of higher pulse energy, absorption rate and fluctuation of laser energy results in damaged tracks (ablation) visible in SEM images at 8 and $13 \mathrm{MHz}$ (Figure 6.1).
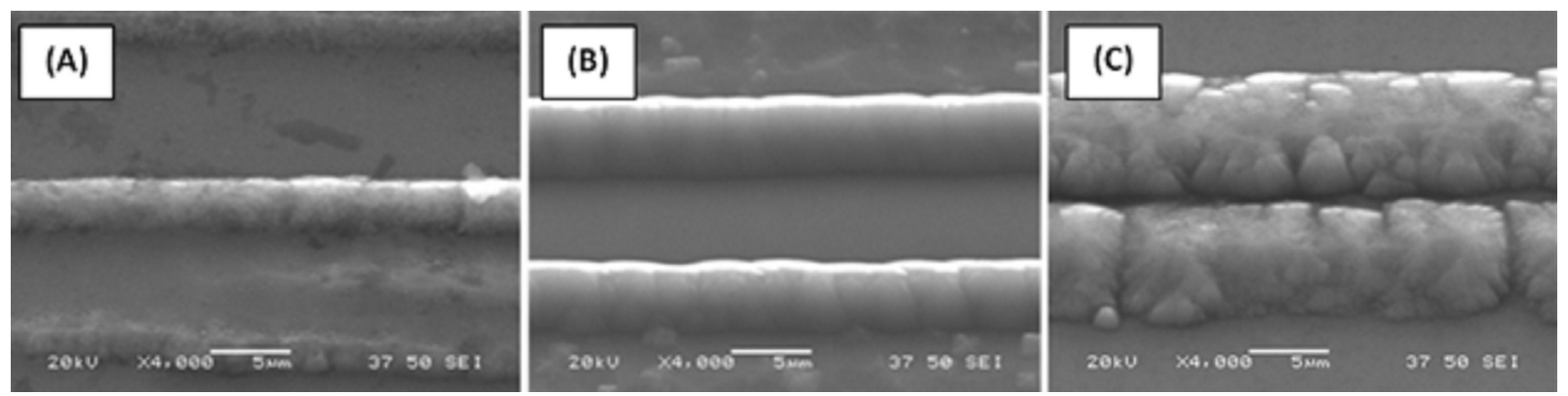

Figure 6.2: SEM images at different pulse width at the scanning speed of $75 \mathrm{~mm} / \mathrm{s}$, pulse repetition of 26 $\mathrm{MHz}$ and the power of $6 \mathrm{~W}$, (a) $214 \mathrm{fs} 4.7 \mu \mathrm{m}$, (b) $720 \mathrm{fs}, 5.3 \mu \mathrm{m}$, and (c) $3500 \mathrm{fs}, 7.2 \mu \mathrm{m}$

\begin{tabular}{|c|c|c|}
\hline Rep. (MHz) & Power (W) & Pulse Fluence $\left(\mathrm{J} / \mathrm{cm}^{2}\right)$ \\
\hline 8 & 4.8 & 0.71 \\
\hline 13 & 5.6 & 0.51 \\
\hline 26 & 6.25 & 0.28 \\
\hline
\end{tabular}

Table 6.2: Laser fluence at different powers and repetition rates at the pulse duration of 214 fs

\subsubsection{Effect of pulse duration}

To indicate how the width of the amorphorized area changes with pulse duration, Figure 6.2 and Figure 6.3 show the width of the amorphorized zone for a range of pulse duration from $214 \mathrm{fs}$ to $3500 \mathrm{fs}$ at the constant power and repetition rate. According to Woodard and Dryden [41,48], for a constant amount of energy, an increase in the pulse width corresponds to a decrease in the temperature distribution, since for infinitesimally short pulse duration 
all the energy would be injected to the target at once, leading to extremely high surface temperature. However, if a constant amount of power is applied, then it would be expected that the longer the pulse duration, the larger the temperature distribution [48]. It can be concluded for the case of constant power, the Heated Affected Zone (HAZ) became wider for increased pulse duration; thus, the width of the amorphorized area became larger at longer pulse duration.

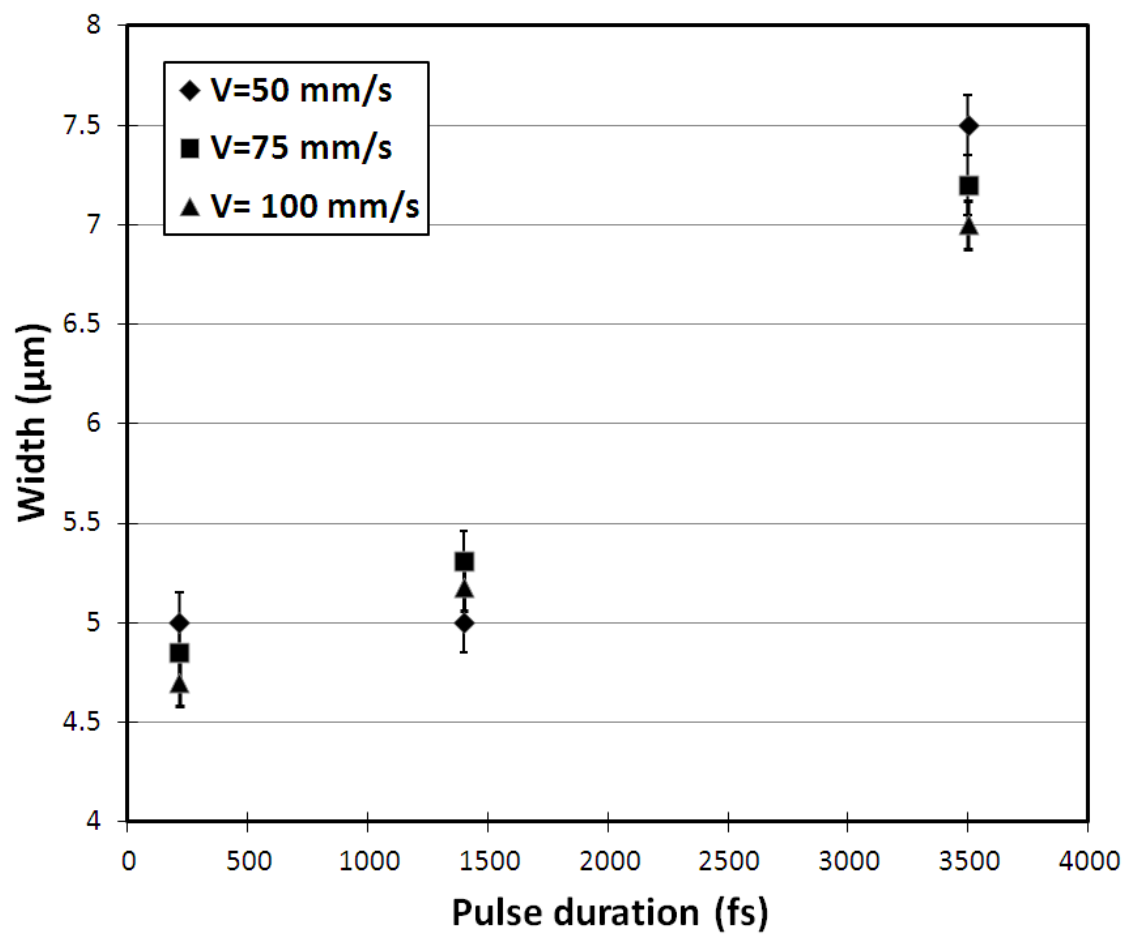

Figure 6.3: Width of amorphorized zone vs pulse width for $214 \mathrm{fs}, 720 \mathrm{fs}$, and $3500 \mathrm{fs}(26 \mathrm{MHz})$

To verify the accuracy of the experimental results, analytical methods were used to study the effects of pulse duration on the amorphization process. For cylindrical symmetric flow without internal heat generation and constant, isotropic material properties, the transient heat conduction can be calculated by: 


$$
\frac{1}{r} \frac{\partial}{\partial r}\left(r \frac{\partial T}{\partial r}\right)+\frac{\partial^{2} T}{\partial z^{2}}=\frac{1}{\alpha} \frac{\partial T}{\partial t}
$$

The boundary condition can be given by:

$$
\left.\frac{\partial T}{\partial z}\right|_{z=0}=-\frac{1}{k} \frac{\eta P(t)}{\pi r_{0}^{2}} \exp \left(-r^{2} / r_{0}^{2}\right) \text { for } t \leq t_{p}
$$

where $P$ is the peak power of the laser pulse which is a function of time, $\eta$ is the fraction of the surface absorption, $t_{p}$ is pulse width and $r_{0}$ is the diameter of the beam spot.

It is convenient to introduce nondimensional groups as follows [48]:

$$
\begin{aligned}
& R=r / r_{0}, \\
& Z=z / r_{0}, \\
& \Theta=T / T_{r e f}, \\
& \tau=4 \alpha t / r_{0}^{2}
\end{aligned}
$$

Thus:

$$
\frac{1}{R} \frac{\partial}{\partial R}\left(R \frac{\partial \theta}{\partial R}\right)+\frac{\partial^{2} \theta}{\partial Z^{2}}=4 \frac{\partial \theta}{\partial \tau}
$$

The boundary condition:

$$
\left.\frac{\partial \theta}{\partial Z}\right|_{z=0}=-Q \exp \left(-R^{2}\right)\left[u(\tau)-u\left(\tau-\tau_{p}\right)\right]
$$


The nondimensional temperature in the integral form can be estimated by:

$$
\theta(R, Z, \tau)=\frac{Q}{2 \sqrt{\pi}} \int_{\tau-\tau_{p}}^{\tau} I(\nu) d \nu
$$

where,

$$
I(\nu)=\left(\frac{1}{1+\nu} \exp \left(-\frac{R^{2}}{1+\nu}-\frac{Z^{2}}{\nu}\right)\right)
$$

Here, $I$ is absorbed laser energy and $\nu$ is the volume of irradiated material. The maximum surface temperature which occurs at the end of the laser pulse on the substrate can be approximately calculated by:

$$
\theta\left(R, Z=0, \tau_{p}\right) \approx \frac{Q}{\sqrt{\pi}} \arctan \left(\sqrt{\tau_{p}}\right)-Q Z
$$

The maximum non-dimensional surface temperature which occurs at the center of the laser beam is given by:

$$
\theta_{\max }(\tau)=\frac{Q}{\sqrt{\pi}}\left[\arctan (\pi)-\arctan \left(\sqrt{\tau-\tau_{p}}\right)\right]
$$

For $\tau<\tau_{p}: \theta(0,0, \tau)=\frac{Q}{\sqrt{\pi}} \sqrt{\tau}$.

Amorphization will begin at the melting temperature of silicon, which is taken to be around $1400{ }^{\circ} K\left(T_{m}\right)$. It is assumed that amorphization happens instantly at the melting temperature because of the rapid cooling rate of silicon after the laser irradiation (up to $10^{13}{ }^{\circ} \mathrm{C} / \mathrm{s}$ ) 


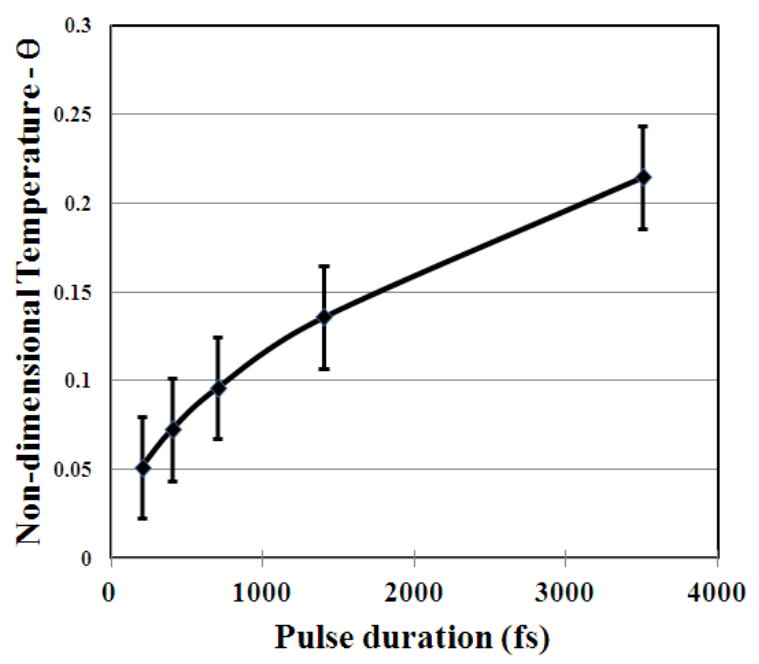

(a)

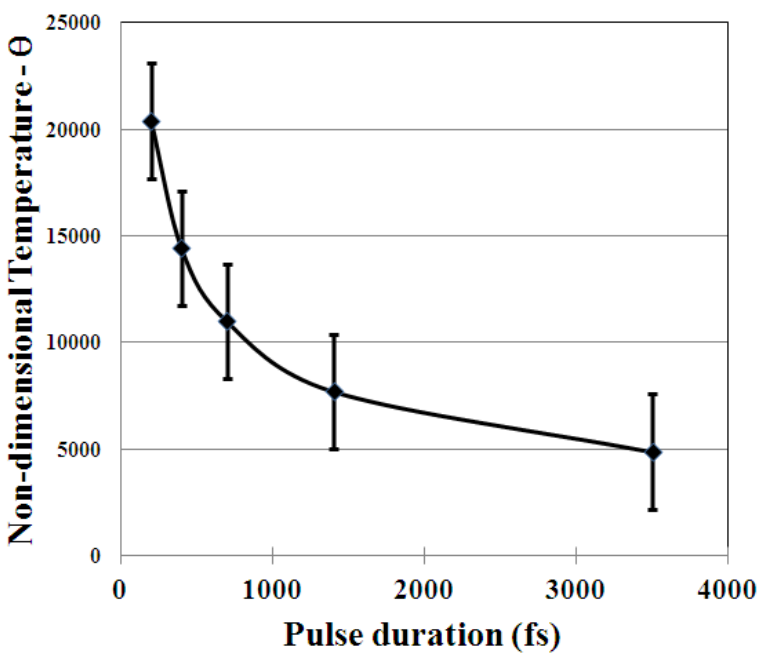

(b)

Figure 6.4: Computed non-dimensional surface temperature at constant amount of a) power and b) energy (b)

which means there is not enough time for the melted silicon to be re-solidified into a crystalline state $[47,49,65]$. The volume where the temperature reaches the amorphization temperature $\left(T_{a}\right)$ is assumed to fully transform to amorphorized silicon by the end of the cycle. Figure 6.4a and Figure 6.4b, show the computed results of nondimensional surface temperatures with constant average laser pulse power (Figure 6.4a) and energy (Figure 6.4b). If a constant amount of power is applied, nondimensional power, $Q$, can be taken arbitrarily $1(Q=1)$. It was found from the results shown in Figure 6.4a that an increase in the pulse width results in an increase in the surface temperature.

On the other hand, for a constant amount of energy $\left(Q \times \tau_{p}=\right.$ const. or $\left.Q=\frac{1}{\tau_{p}}\right)$, the energy would be applied at each pulse and the shorter pulse duration results in larger surface temperature (Figure 6.4b).

Table 6.3 shows the different results of Raman peaks of a-Si (at $\left.480 \mathrm{~cm}^{-1}\right)$, an increase in 


\begin{tabular}{|c|c|c|l|l|l|l|}
\hline No. & $\begin{array}{l}\text { Pulse dur- } \\
\text { ation }(\mathrm{fs})\end{array}$ & $\begin{array}{l}\text { Power } \\
(\mathrm{W})\end{array}$ & $\begin{array}{l}\text { Non- } \\
\text { dimensional } \\
\text { pulse duration } \\
-\tau\end{array}$ & $\begin{array}{l}\text { Non- } \\
\text { dimensional } \\
\text { power - Q }\end{array}$ & $\begin{array}{l}\text { Non- } \\
\text { dimensional } \\
\text { temperat- } \\
\text { ure }-\Theta\end{array}$ & $\begin{array}{l}\text { Raman peak of } \\
\text { a-Si at 480 } \mathrm{cm}^{-1} \\
(\text { a.u. })\end{array}$ \\
\hline 1 & 200 & 8 & $2.514 \times 10^{-6}$ & 1.2192 & 0.06250 & 23.125 \\
\hline 2 & 400 & 7.5 & $5.030 \times 10^{-6}$ & 1.1430 & 0.08288 & - \\
\hline 3 & 700 & 7.2 & $8.802 \times 10^{-6}$ & 1.0973 & 0.10526 & 42.675 \\
\hline 4 & 1400 & 5.4 & $17.604 \times 10^{-6}$ & 0.8230 & 0.11165 & 64.75 \\
\hline 5 & 3500 & 5.2 & $44.001 \times 10^{-6}$ & 0.7930 & 0.17008 & 97.8 \\
\hline
\end{tabular}

Table 6.3: Analytical and experimental results of the amorphorized silicon wafers

non-dimensional temperatures for different values of the experiments.
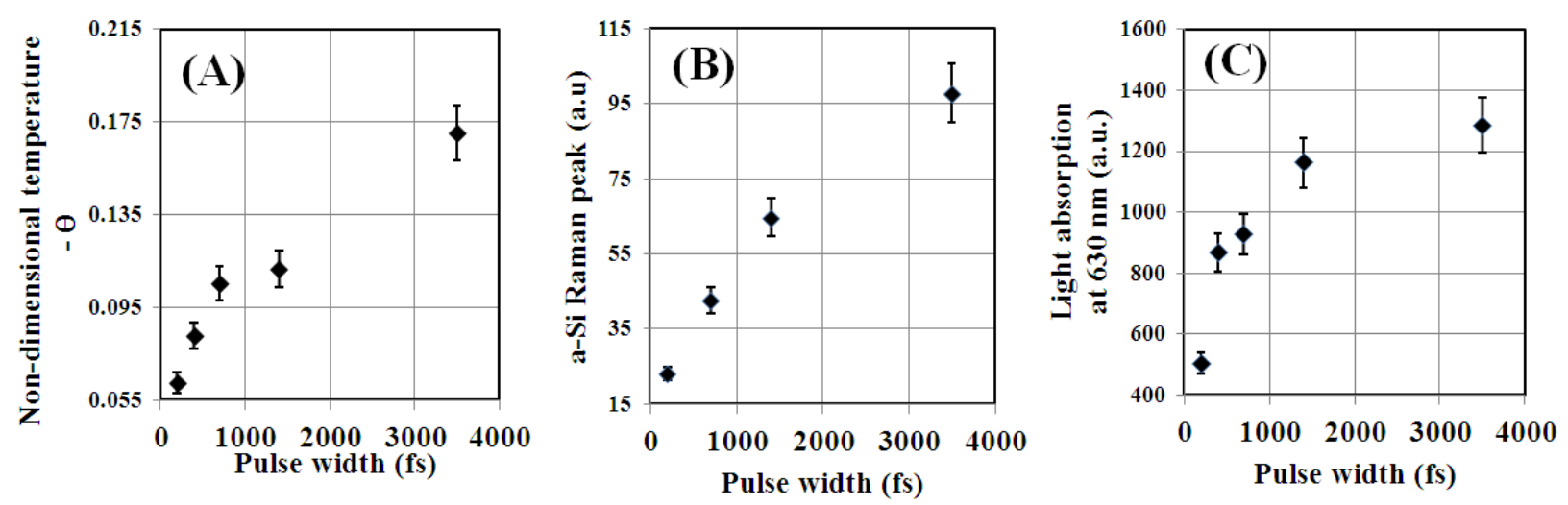

Figure 6.5: Analytical/experimental graphs of: a) non-dimensional surface temperature, b) raman peak of amorphous silicon, c) light absorption at $630 \mathrm{~nm}$

\subsubsection{Effect of number of laser pulses}

\subsubsection{Effect of number of laser pulses on target surface temperature}

The analytical/experimental results of the experiment for different parameters such as nondimensional temperature, micro-Raman peaks of amorphorized silicon and light absorption 
of irradiated samples for different pulse widths and laser power levels are shown in Figure 6.5. The computed results in Figure 6.5(a) show that by increasing the pulse duration (and decreasing the laser power at the same time) the surface temperature of the irradiated area increased, which means that more energy was injected into the $\mathrm{Si}$-substrate; thus, the HAZ, or the volume where the temperature reaches the amorphization temperature, became wider; it resulted in an increase of the amount of silicon converted from a crystalline to amorphorized state as shown in the micro-Raman spectroscopy for the amorphorized silicon peak at $480 \mathrm{~cm}^{-1}$ (Figure 6.5(b)). Moreover, it was found that the light-absorption of the amorphorized sample improved by irradiation with longer laser pulses. Although the calculated results are not actual parameters (nondimensional) and they may contain errors due to the assumptions, the tendency of the surface temperature, amorphization rate and improvement in light absorption in relation to pulse duration is quite clear and this can be used to explain the phenomenon observed in experimental results [49].

After being irradiated by the femtosecond laser with variable scanning speed, the samples were observed under a SEM. Figure 6.6 shows that the width of amorphous zones changed with the various irradiation scanning speeds. The effective number of pulses, $N_{\text {eff }}$, can be calculated from scanning speed, $\nu$, by $N_{\text {eff }}=\sqrt{\pi 2 \omega_{0}} f / \nu$. Corresponding to $25,50,75$ and $100 \mathrm{~mm} / \mathrm{s}$, the numbers of pulses are 10712, 5356, 3570 and 2678, respectively. In fact, the increase in the pulse numbers leads to an increase in the width of amorphorized lines. As shown in Figure 6.7, the experimental results support such a conclusion. At the same average input power of $7.2 \mathrm{~W}$, the width of the amorphorized zone enlarged with the incident pulse number increasing. As the incident pulse number increases, more energy was deposited; therefore, the heat affected areas would expand [41,72]. 


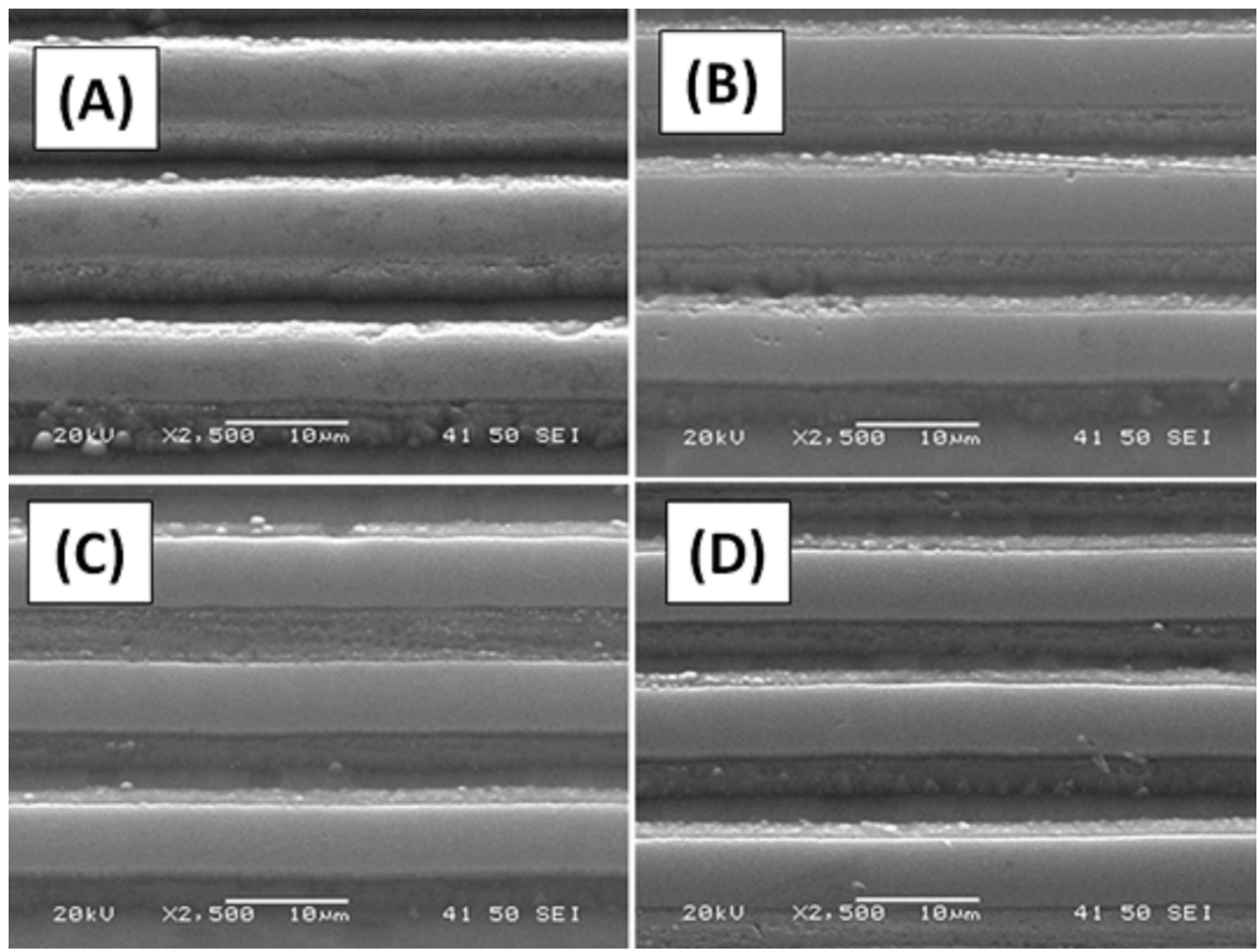

Figure 6.6: SEM images of silicon samples after irradiated with 3500 fs laser at different number of pulses (26 MHz), (a) $25 \mathrm{~mm} / \mathrm{s}$, (b) $50 \mathrm{~mm} / \mathrm{s}$, (c) $75 \mathrm{~mm} / \mathrm{s}$, and (d) $100 \mathrm{~mm} / \mathrm{s}$

Furthermore, analytical and theoretical methods were used to investigate the effects of laser parameters. In the analyses for calculating the surface temperature after the end of the laser pulse, $\Delta T$, at the center of the spot area can be approximately calculated by $[38,39]$ :

$$
T(0, t)=T\left(0, t_{p)}\left(\frac{t_{p}}{t}\right)^{1 / 2}=\sqrt{2 / \pi} \frac{I_{a}\left(a t_{p}\right)^{1 / 2}}{k}\left(\frac{t_{p}}{t}\right)^{1 / 2}\right.
$$

where, $t_{p}$ is pulse duration, $a$ is the thermal diffusion coefficient and $k$ is the heat conduction coefficient and $I_{a}$ is the absorbed laser light intensity, which is estimated by the residual 


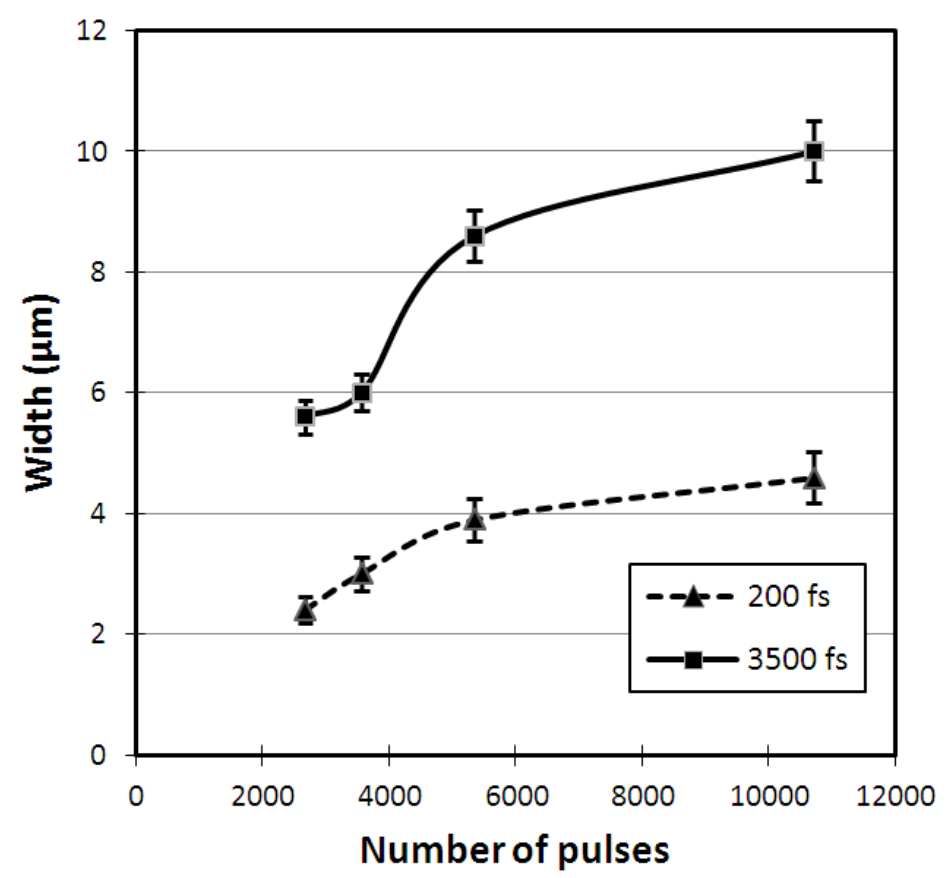

Figure 6.7: Width of amorphorized zone vs number of pulses for 2000 to 11000 (26 MHz)

energy coefficient, $K$, and the reflection coefficient, $R$, at a wavelength of $1030 \mathrm{~nm}$ as follows $[51,61]:$

$$
I_{a}=K(1-R) \frac{4 P}{\pi d^{2} t_{p} f}
$$

where $P$ is laser power, $f$ is pulse frequency, and $d$ is laser spot diameter. Substituting Equation 6.1 in Equation 2.6, it is simplified to:

$$
T\left(0, t_{p}\right)=\sqrt{\frac{2 a}{\pi^{3} t_{p}}} \frac{4 K(1-R) P}{k f d^{2}}
$$

The reflection coefficient of silicon at a wavelength of $1030 \mathrm{~nm}$ is calculated to be around 
0.325 [62]. Experiments showed that $K$ for silicon is around 0.8 when the fluence is less than the ablation threshold [63]. In the experiment, the thermal conductivity $k$ is $155 \mathrm{~W} /(\mathrm{mK})$ and the thermal diffusivity is $8.5 \times 10^{-5} \mathrm{~m}^{2} / \mathrm{s}$ [64]. The maximum surface temperature at the end of the laser pulse is $T_{\max }$ or $T_{m}=T\left(0, t_{p}\right)$, and $T_{\min }$ is the minimum temperature at the beginning of the following laser pulse, which is given by $T_{\min }=\alpha T_{\max }$. Here $\alpha$ is the constant ratio for the previous maximum and the following minimum temperatures and equal to $\alpha=\left(t_{p} / t_{p p}\right)^{1 / 2}$, where $t_{p p}$ is the pulse interval and equal to $t_{p p}=1 / f$ ( $f$ is pulse repetition rate) and $t_{p}$ is pulse duration. From the mentioned relationships, the maximum and minimum surface temperature of irradiated area can be approximately calculated as $[38,39]$ :

First pulse:

$$
\begin{aligned}
& \left(T_{\max }\right)_{1}=T_{m} \\
& \left(T_{\min }\right)_{1}=\alpha T_{m} ;
\end{aligned}
$$

Second pulse:

$$
\begin{aligned}
& \left(T_{\min }\right)_{2}=\alpha(1+\alpha) T_{m} \\
& \left(T_{\max }\right)_{2}=(1+\alpha) T_{m}
\end{aligned}
$$

$n$-th pulse:

$$
\begin{aligned}
& \left(T_{\max }\right)=\left(1+\alpha+\alpha^{2}+\alpha^{3}+\ldots+\alpha^{n-1}\right) T_{m}=\left[\left(1-\alpha^{n}\right) /(1-\alpha)\right] T_{m} \\
& \approx(1-\alpha)^{-1} T_{m}\left(T_{\min }\right)_{n}=\alpha\left(T_{\max }\right)_{n}
\end{aligned}
$$

The surface temperature average over any $i$-th laser pulse and the time gap between the $i$-th and the $(i+1)$-th is given by: 


$$
\bar{T}_{i}=\frac{1}{t_{p}+t_{p p}} \int_{0}^{t_{p}}+t_{p p} T_{m, i}(0, t) d t=2 \alpha T_{m, i} \frac{\left(1-\frac{2}{3} \alpha\right)}{\left(1+\alpha^{2}\right)}
$$

Finally, the average surface temperature after $n$ pulses can be calculated by:

$$
\bar{T}_{n}=2 \alpha \frac{\left(1-\frac{2}{3} \alpha\right)}{\left(1+\alpha^{2}\right)} \frac{T_{m}}{(1-\alpha)}\left(1+\frac{\alpha^{n}-\alpha}{n(1-\alpha)}\right)
$$

In the case $n \gg 1$ and $\alpha \ll 1$ :

$$
\bar{T}_{n} \cong 2 \alpha T_{m}=2 T_{m}\left(\frac{t_{p}}{t_{p p}}\right)^{1 / 2}
$$

The computed results are shown in Figure 6.8. Initially, the surface temperature increases with the accumulation of number of pulses. After a certain number of pulses, the surface temperature reaches saturation. Further increase in the number of pulses does not lead to a significant rise in the surface temperature. This computation shows that at a constant laser power of $5.6 \mathrm{~W}$, the final surface temperature at $13 \mathrm{MHz}$ is around $932^{\circ} \mathrm{C}$, compared to $1196^{\circ} \mathrm{C}$ at $8 \mathrm{M} \mathrm{Hz}$. The final surface temperature at $26 \mathrm{M} \mathrm{Hz}$ at a higher laser power of $6.5 \mathrm{~W}$ is $770^{\circ} \mathrm{C}$. Although the calculated temperature may contain the large error due to the assumptions, the tendency of the surface temperature in relation to the repetition rate is quite clear, and this can be used to explain the phenomenon observed in Figure 6.1 and Figure 6.6. At a constant laser power, the surface temperature at $8 \mathrm{MHz}$ and $13 \mathrm{MHz}$ is marginally below the melting point of silicon. Therefore, a slight deviation in pulse energy will result in the surface temperature rising above the threshold for material removal. It 
(a) $6.5 \mathrm{~W}, 26 \mathrm{MHz}$

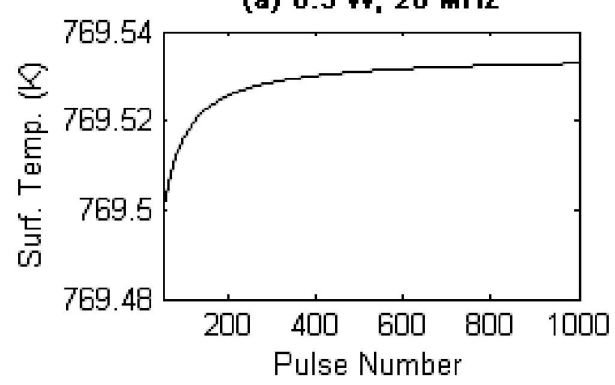

(c) $5.6 \mathrm{~W}, 13 \mathrm{MHz}$

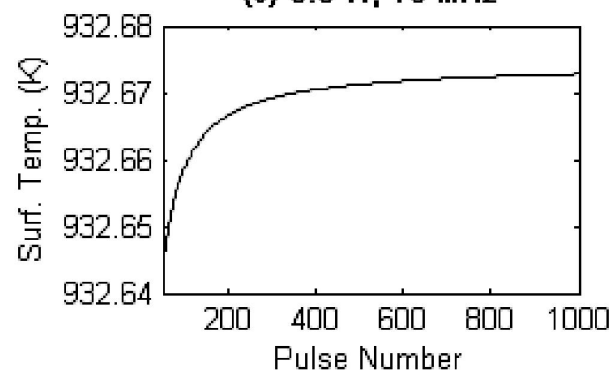

(e) $5.6 \mathrm{~W}, 8 \mathrm{MHz}$

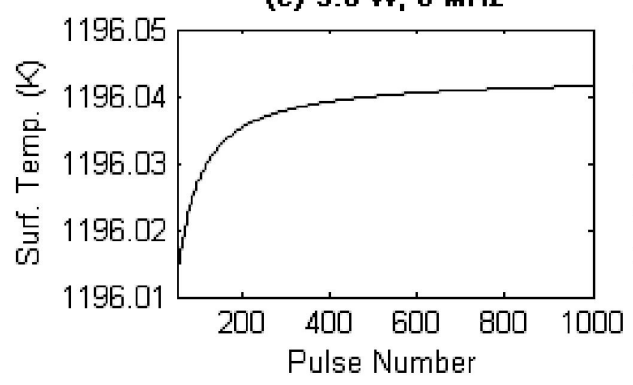

(b) $7.2 \mathrm{w}, 26 \mathrm{mHz}$

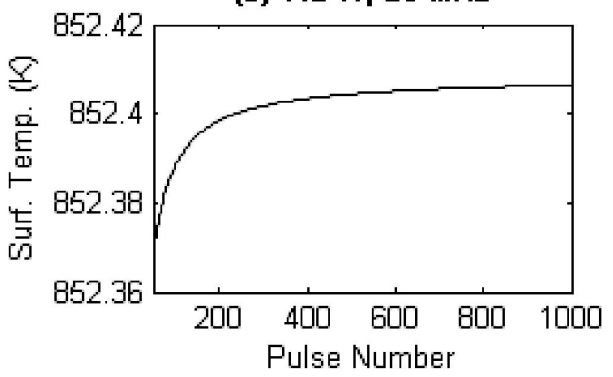

(d) $6.2 \mathrm{w}, 13 \mathrm{mHz}$

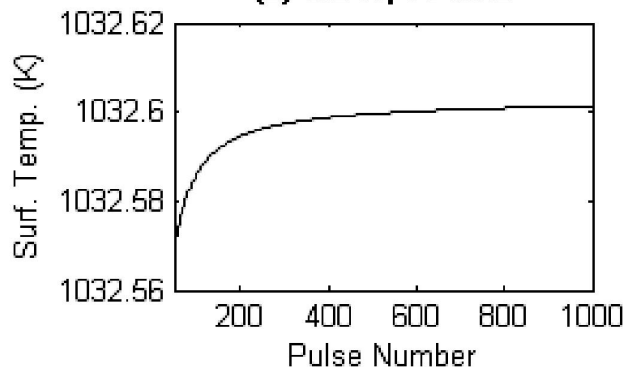

(f) $6.5 \mathrm{~W}, 8 \mathrm{mHz}$

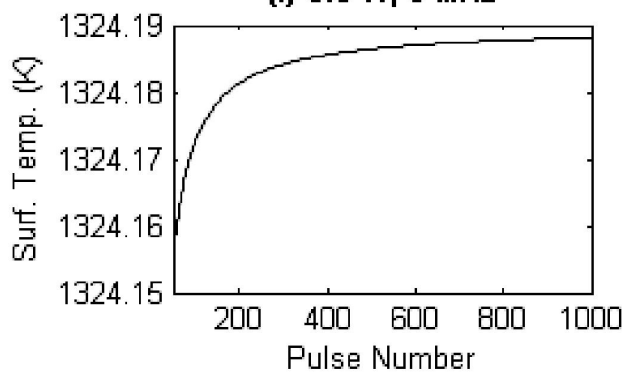

Figure 6.8: Change in surface temperature at different pulse repetitions and powers (pulse width of 214 fs)

can be concluded that the center-line ablated cracks shown in Figure 6.1 are not because of the large number of pulses but the combination of higher pulse energy. In contrast, the final surface temperature at $26 \mathrm{MHz}$ is always maintained below the ablation threshold, preventing the presence of visible damage at the center of the laser focus. The computed results explain the fact that if the laser fluence is kept below the ablation threshold, no ablation occurs no matter how many pulses irradiate the silicon substrate. This could be 
attributed to the heat diffusion in between successive pulses [38, 39, 41, 54]. It is important to note that although the number of pulses incident on the silicon surface does not lead to the increased surface temperature up to the ablation, the width of the amorphorized zone becomes wider with increasing number of pulses [41].

\subsubsection{Effect of number of laser pulses on the amorphization threshold}

As indicated previously by researchers $[71,73]$, for lasers with the Gaussian beam profile, the feature size can be calculated as follows:

$$
D^{2}=2 \omega_{0}^{2}\left\{\ln \phi_{0}-\ln \phi_{t h}(N)\right\}
$$

where, $\omega_{0}$ is the radius of the laser spot, $\phi \operatorname{th}(\mathrm{N})$ is the multi-pulse modification (amorphization) threshold, which depends both on the number of pulses and material, and $\phi_{0}$ is the maximum laser fluence which is given by:

$$
\phi_{0}=\frac{2 E_{\text {pulse }}}{\pi \omega_{0}^{2} f}
$$

From Equation 6.3 and Equation 6.4, the multi-pulse amorphization threshold can be estimated as [41]:

$$
\phi_{t h}(N)=\frac{2 P}{\pi \omega_{0}^{2} f \exp \left[0.5\left(D / \omega_{0}\right)^{2}\right]}
$$

Figure 6.9 shows the calculated results of $\phi \operatorname{th}(\mathrm{Ns})$ based on the analytical solution combined 
with the experimental results for the width of the amorphorized zone for different numbers of pulses. As shown in Figure 6.9, the n-pulse amorphization threshold decreases with the

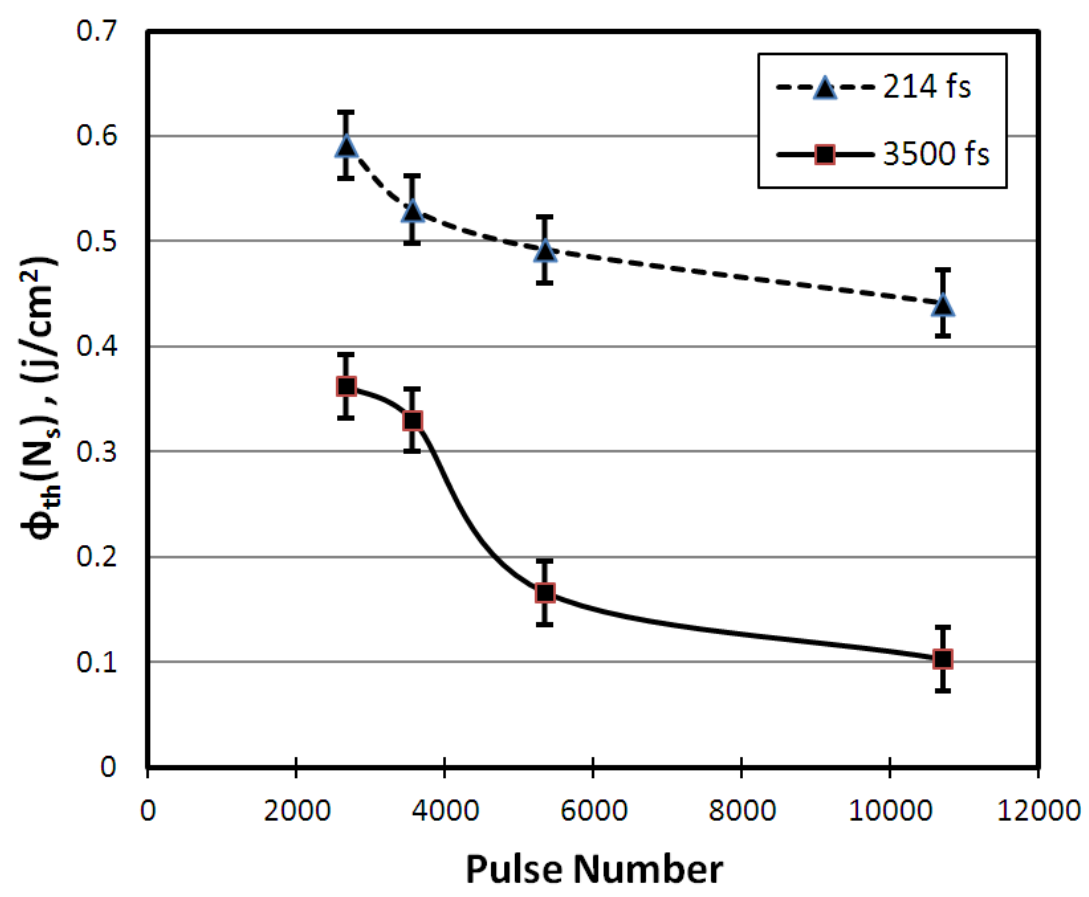

Figure 6.9: $\phi(N)$ vs number of pulses $(26 \mathrm{MHz})$

increase in the number of pulses. However, beyond 5000 pulses the amorphization threshold nearly reaches saturation. This result agrees well with previous results obtained for the ablation threshold $[38,41,71,73]$.

Table 6.4 tabulates the measured amorphization line width at various repetition rate. It is worth mentioning that even though the relative fluence (pulse fluence $/ \phi_{t h}(\mathrm{~N})$ ) is almost constant (2. 35-2.1) at all repetition rates, the line width is larger at lower repetition rates, which indicates that the dominating factor for line width is pulse energy. 


\begin{tabular}{|l|l|l|l|l|l|l|}
\hline $\begin{array}{l}\text { Rep. } \\
(\mathrm{MHz})\end{array}$ & Power $(\mathrm{W})$ & $\begin{array}{l}\text { Pulse } \\
\text { Fluence } \\
\left(\mathrm{J} / \mathrm{cm}^{2}\right)\end{array}$ & $\begin{array}{l}\text { Speed } \\
(\mathrm{mm} / \mathrm{s})\end{array}$ & $\begin{array}{l}\text { Pulse } \\
\text { Number }\end{array}$ & $\begin{array}{l}\text { Width } \\
(\mu \mathrm{m})\end{array}$ & $\begin{array}{l}\phi_{t h}(\mathrm{~N}) \\
\left(\mathrm{J} / \mathrm{cm}^{2}\right)\end{array}$ \\
\hline 8 & 4.8 & 0.71 & 100 & 900 & 5.80 & 0.302 \\
\hline 13 & 5.6 & 0.51 & 100 & 1350 & 4.40 & 0.231 \\
\hline 26 & 6.25 & 0.28 & 100 & 2700 & 4.05 & 0.131 \\
\hline
\end{tabular}

Table 6.4: $\phi_{t h}(\mathrm{~N})$ at different powers and repetition rates (pulse duration: $214 \mathrm{fs}$ ).

\subsection{Summary}

In this chapter, the effect of laser parameters (repetition rate, number of pulses, pulse duration) on the quality of amorphorized silicon induced by high repetition femtosecond laser pulses and hence the size of the amorphorized feature has been studied. The experimental results outlined in this report demonstrate that the quality and controllability of amorphization of silicon would be better at the higher repetition rate. It was also found that with shorter pulse duration the width of the amorphorized zone became smaller as well.

A theoretical model of surface temperature has been developed to investigate the effect of the number of pulses on the induced amorphorized area. From the experimental and theoretical results, it was found that at constant laser power, surface temperature is low at higher repetition rate, which is favorable for amorphization. However, with the increase in the number of pulses, the width of amorphorized zone becomes larger and the n-pulse amorphization threshold decreases as well [41].

Furthermore, a theoretical model of nondimensional surface temperatures has been used to investigate the effect of the pulse width on an induced amorphorized thin layer on $\mathrm{Si}$-substrate, which was in good agreement with experimental results. Using high repe- 
tition laser pulses with the different pulse durations in the range of $200-3500 \mathrm{fs}$ enabled us to control the laser fluence in the amorphization range, and finally a thin film of amorphorized silicon was fabricated on $\mathrm{Si}-$ substrate [49]. 


\section{Chapter 7}

\section{Application of}

\section{Amorphorized/Oxidized Silicon}

\section{Thin-film}

I

$\mathrm{N}$ this chapter, the feasibility of using the proposed technique as a solution for maskless lithography will be carried out. The proposed approach enables a direct (single-step) generation of an oxide/amorphous layer on a silicon substrate which also can act as an etch stop in the wet etching process. The direct oxidation/amorphization of silicon induced by the femtosecond laser is a maskless single-step technique which offers a higher flexibility and reduced processing time. In addition this method allows for large-area patterning (in mm scale) at fast writing speed under ambient condition.

Moreover, in this chapter, the feasibility of using a-Si thin film generated by the proposed 
method for solar cell fabrication is investigated. In this method, controlled laser fluence, which is injected into the silicon substrate, causes the thin layer of crystalline Si-substrate to be converted to a thin film of amorphous silicon. This method is single step and does not need to have additive materials; in comparison with current $a-S i$ thin-film solar cell fabrication methods, both processing time and wafer damage are significantly reduced in this method.

\subsection{Maskless Lithography Using Oxidized/Amorphorized Silicon Etch-stop Layer Induced by Mega Hertz Repetition Femtosecond Laser Pulses}

Photolithography is considered to be an important method in a wide range of applications such as fabrication of information storage media, micro and nano photonics, nanoelectromechanical systems (NEMS), microelectromechanical systems (MEMS), microfluidics and LOC Systems $[3,5-9,15]$.

Patterning layers of etch stop materials on substrates (such as silicon) is one of the most important issues in the photolithography process. Silicon oxide is one of the most attractive materials which is used as an etch stop during etching processes. In contrast to other etch stop materials which suffer from one or more problems, silicon oxide offers a lot of desired characteristics and advantages which make silicon oxide an evermore-valuable compound in the photolithography method. Known methods for the generation of silicon dioxide $\left(\mathrm{SiO}_{2}\right)$ patterns generally involve either physical or chemical vapor deposition; the deposited films 

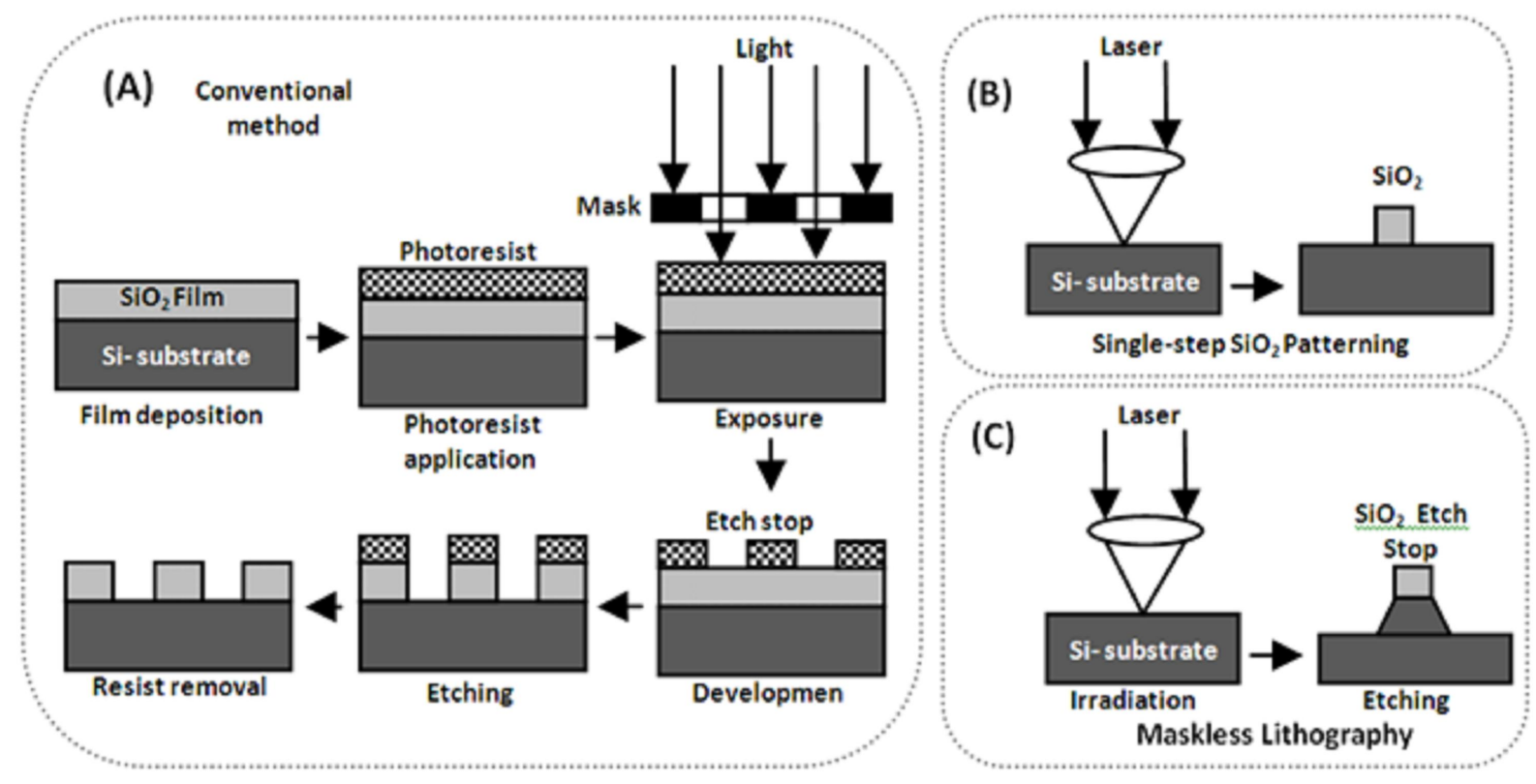

Figure 7.1: a) conventional lithography method, b) direct (single-step) silicon oxide patterning, c) direct maskless lithography induced by fs laser

must be patterned subsequently using a resist and various etching techniques which are often accomplished through multi-step fabrication methods [51,74-80]. Additionally, conventional photolithography technique is not well-suited to cost-effective, and high-throughput processing since there are several steps in the fabrication process and this technique requires a photomask for replication and its fabrication is time consuming and expensive $[38,81,82]$. Over the past few years, a number of novel techniques such as Scanning Electron Beam Lithography (SEBL) [16,83,84], Focused Ion Beam (FIB) [17,85], multi-axis electron beam lithography (MAEBL) [86], Interference Lithography (IL) [87,88], Maskless Optical-Projection Lithograph (MOPL) [89], Easy Soft Imprint Nanolithography (ESINL) [90], Scanning-Probe Lithography (SPL), DPL [91,92], and TNL based on AFM [21,22,25,27] have been reported for maskless replication of micro/nano scale patterns of silicon oxide on silicon substrates. 
This chapter attempts to use this oxidized/amorphorized layer as an etch stop in alkaline etchant, such as $\mathrm{KOH}$ to develop a unique approach for single step maskless lithography by a combination of direct-write femtosecond laser oxidation/amorphization of silicon (etch stop layer) and chemical wet etching. Using megahertz frequency femtosecond laser pulses makes it possible to control laser fluence below ablation threshold which causes crystalline silicon conversion to an oxidized or amorphorized silicon. The induced thin layer of oxidized/amorphorized silicon is then used as an etch stop in the following wet chemical etching (e.g. $\mathrm{KOH}$ ) for maskless lithography. In comparison with previous methods, this method can be a promising solution for both direct generation of silicon oxide or amorphous silicon pattern on Si-substrate (Figure 7.1(b)) and maskless lithography (Figure 7.1(c)); the direct oxidation/amorphization of silicon (etch-stop layer) induced by femtosecond laser is a maskless single-step technique which offers a higher flexibility and reduces the processing time. In addition this method allows for the large-area patterning (in mm-scale) at fast writing speed under ambient conditions. In comparison to systems such as AFM, FIB and Electron Beam (EB), which are employed in other techniques, lasers are much more economical and can be acquired and operated at lower cost [93] which makes it particularly suitable for rapid prototyping and custom-scale manufacturing for a wide variety of applications in MEMS, NEMS, fabrication of semiconductor and LOC systems. 


\subsubsection{Maskless lithography using thin-film silicon oxide as an etch stop}

Figure 7.2 shows morphology of the oxidized silicon patterns on Si-substrate after irradiation with sub-ablation threshold femtosecond laser pulses. SEM images show bump lines around $7 \mu \mathrm{m}$ width and $500 \mathrm{~nm}$ height induced with femtosecond laser pulses with a power of $3.3 \mathrm{~W}$ supplied at $26 \mathrm{MHz}$. As shown in Figure 7.2, the embossed lines (made of silicon oxide) can be generated consistently on a silicon surface with carefully controlled laser parameters.

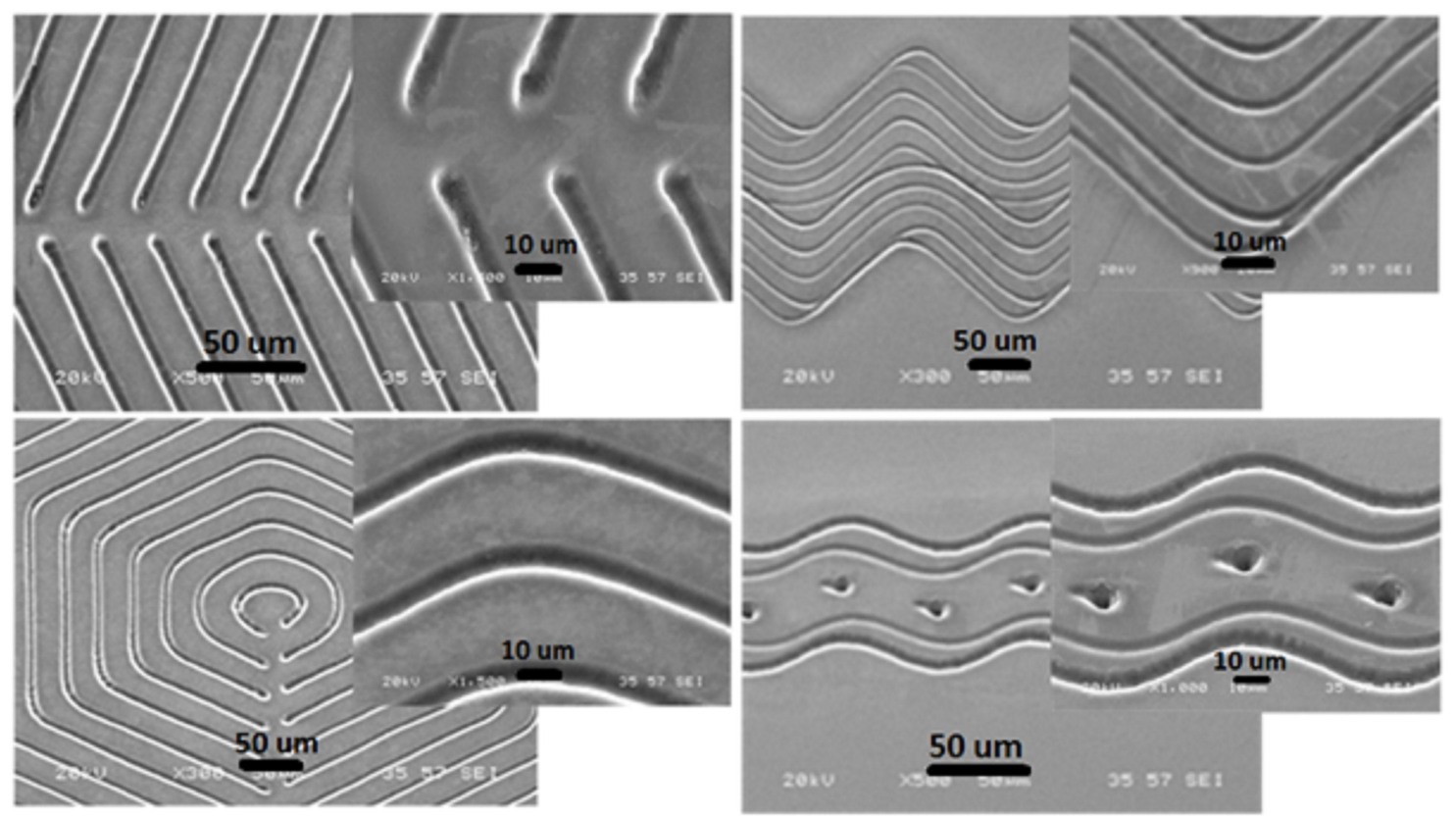

Figure 7.2: SEM images of silicon oxide pattern induced by $214 \mathrm{fs}$ laser pulses at the frequency of $26 \mathrm{MHz}$ and the average power of $3.3 \mathrm{~W}\left(I=0.15 \mathrm{~J} / \mathrm{cm}^{2}\right)$

Generally in the silicon oxidation process, the final silicon oxide layer is approximately $54 \%$ above and $46 \%$ below the original silicon surface which is because of the difference in 
densities between $\mathrm{Si}$ and $\mathrm{SiO}_{2}$ [55]. Figure 7.3 represents optical microscope topography images of the induced areas after irradiation. Cross sectional views show embossed lines around $500 \mathrm{~nm}$ height and $7 \mu \mathrm{m}$ width induced at the laser power of $3.3 \mathrm{~W}$ and frequency of $26 \mathrm{MHz}$ with the pulse duration of $214 \mathrm{fs}$. Due to this fact that the final oxide layer is $54 \%$ above and $46 \%$ below the original surface, it can be concluded that the real thickness of oxide layer on Si-substrate is approximately $1 \mu \mathrm{m}$.

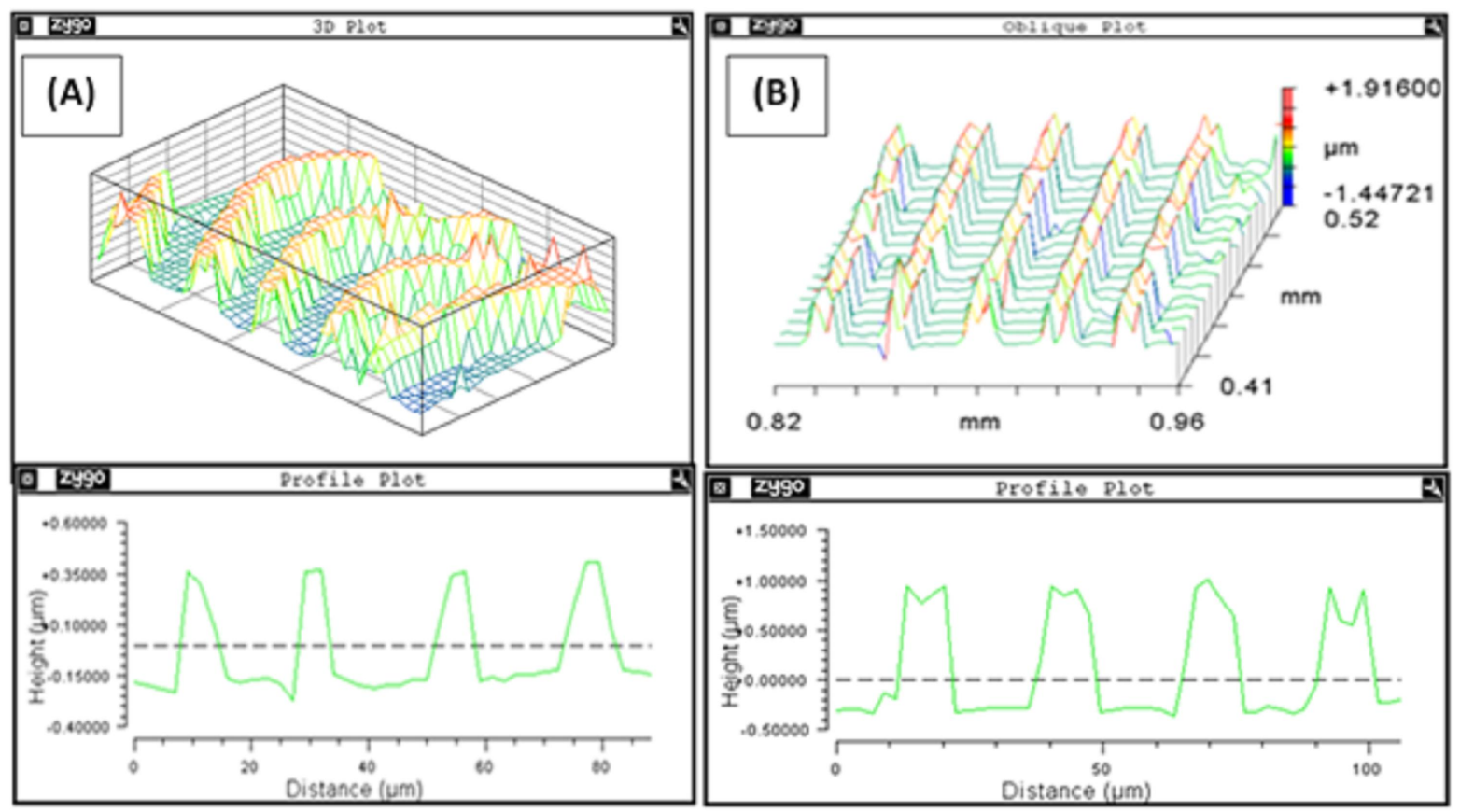

Figure 7.3: Optical microscope topography and cross sectional images (a) after irradiation (height: $500 \mathrm{~nm}$ ) and (b) after etching process (height: $1000 \mathrm{~nm}$ )

In order to verify the elemental composition of the irradiated area (embossed lines), EDX and Raman spectroscopic analyses were employed. EDX results indicated the existence of oxygen in irradiated lines which are explained to be due to the Si-O-Si bonds in an irradiated area (Figure 7.4). 

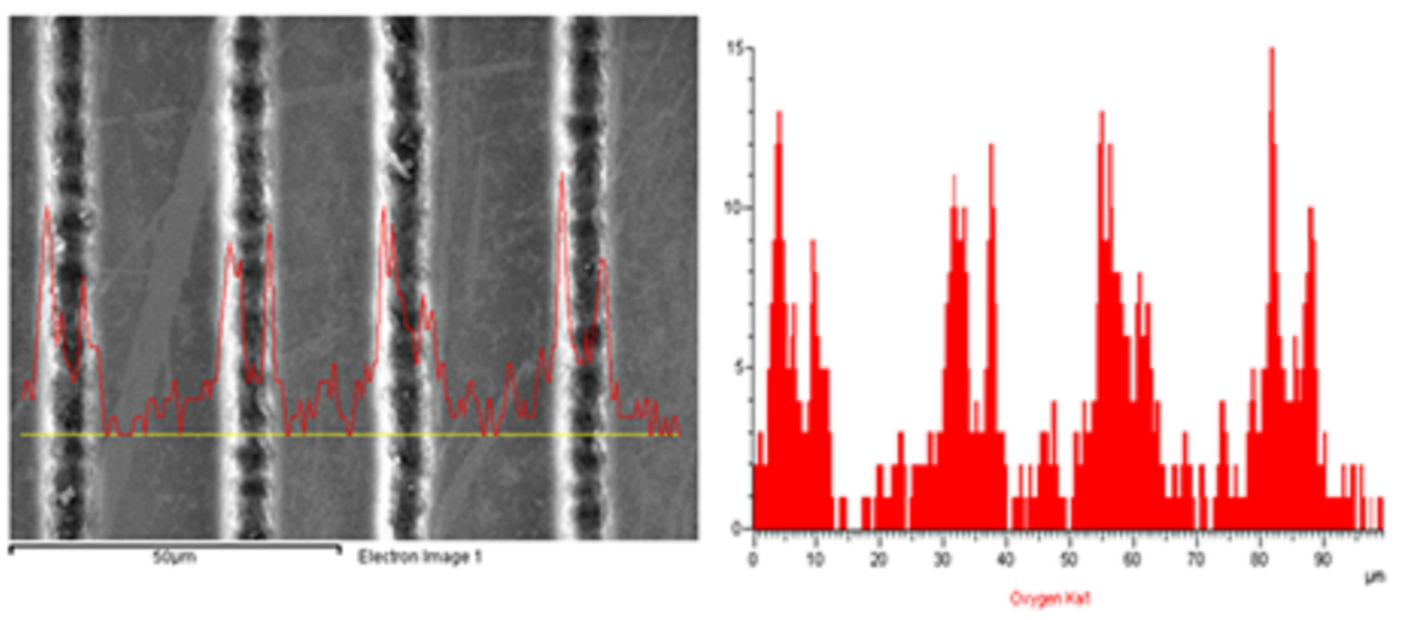

Figure 7.4: EDX results of irradiated area

Raman spectroscopy was dominated by the signal at wavenumber of $519 \mathrm{~cm}^{-1}$ which is the characteristic peak of silicon (Figure 7.5). Furthermore, a hump was observed at the wavenumber of $954 \mathrm{~cm}^{-1}$ corresponding to silicon oxide [37]. Back scattering micro-Raman analysis was performed under ambient conditions using the $532 \mathrm{~nm}$ line of an Ar ion laser source. Consequently, it can be concluded that irradiated lines were converted to silicon oxide.

Figure 7.6 shows XRD data for the silicon samples before and after irradiation by laser. XRD measurement of the samples was performed using an X-ray diffractometer equipped with the X-ray source of $C u$ rotating anode generator $(\lambda=1.54184 \AA)$ operated in a large angle rate of $10^{\circ}-60^{\circ}$. In spite of the good resolution of XRD measurements, the spectra of laser irradiated samples (post-irradiation) exhibits only one crystalline peak and no other crystalline peaks of $S i$ were detected. Moreover, the XRD graph of the laser treated samples (post-irradiation) shifted to the lower level of intensity. Therefore, it can be concluded that the irradiated area converted from a crystalline silicon to an amorphous silicon oxide 


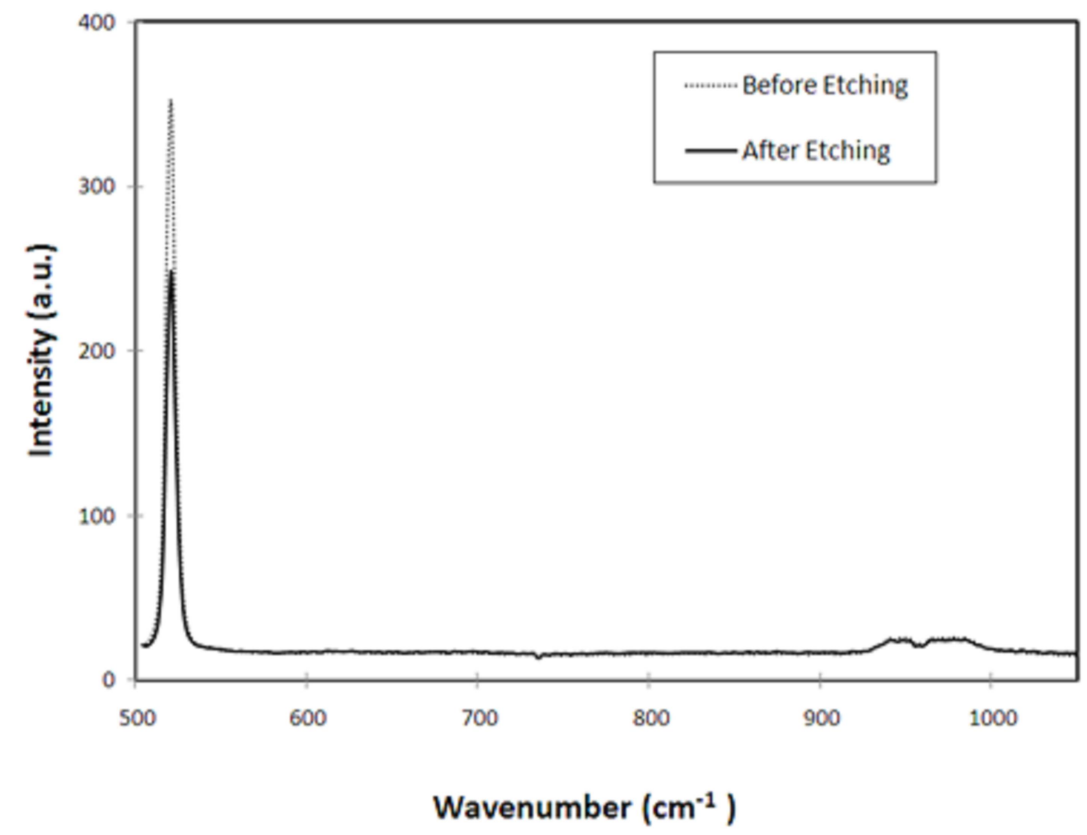

Figure 7.5: micro-Raman spectroscopy results of irradiated samples before/after etching process

$\left(a-S_{i} O_{x}\right)$ which is in agreement with other spectroscopy results obtained by micro-Raman and EDX [54].

The manufacture of many micro/nano scale devices from silicon requires the use of an etch stop layer to selectively stop the anisotropic etching of silicon in chemical etchant such as $\mathrm{KOH}$. Previous studies proposed by researchers have shown that the silicon oxide is an excellent material for creating etch-stop layers on the silicon wafers [74-80]. Additionally, conventional photolithography technique is not well-suited to cost-effective, and high-throughput processing since there are several steps in the fabrication process and this technique requires a photomask for replication and its fabrication is time consuming and expensive $[38,81,82]$. Figure 7.7, shows SEM images of the induced samples after etching in a $30 \% \mathrm{KOH}$ alkaline solution at temperature of $65{ }^{\circ} \mathrm{C}$ after 4 minutes. As shown, the silicon oxidized pattern on 


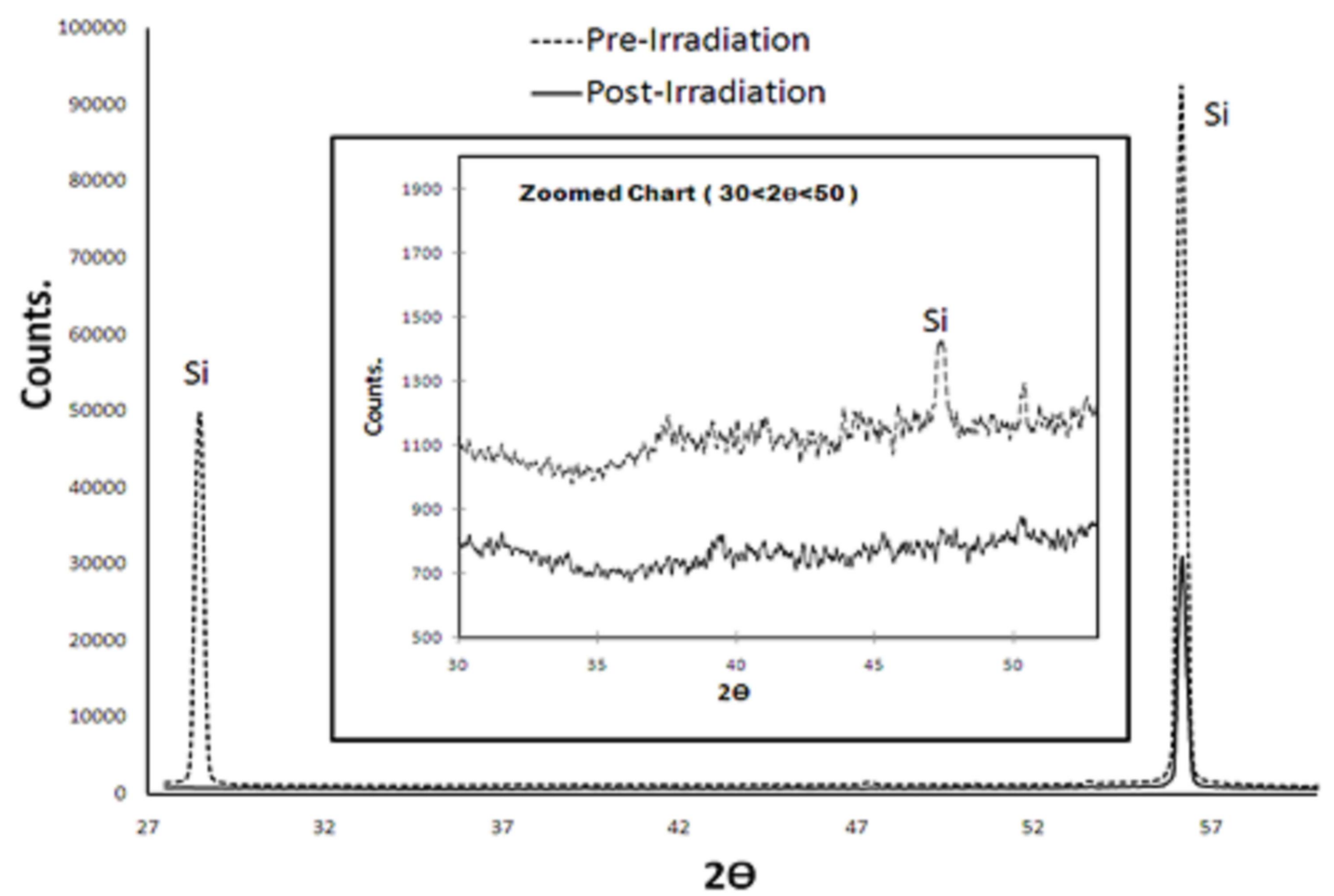

Figure 7.6: XRD results of pre/post-irradiated samples. XRD spectrum of pre-irradiated area is dominated by the signals at $2 \Theta=28,56$ and 47 (zoomed chart) which corresponds to crystalline silicon. The XRD measurements on the processed samples shows only a peak shifted to lower level of intensity at $2 \Theta=56$

silicon substrate acted as an etch stop during the etching process and finally, micro scale features were generated on the silicon substrate. Figure 7.3(b) shows optical microscope topography images of induced Si samples after etching in KOH. As shown in cross sectional view, the height of the generated lines after etching is around $1 \mu \mathrm{m}$ supporting the fact that bumps are made of silicon oxide and act as an etch stop in an alkaline etchant [54].

Figure 7.5 shows the micro-Raman spectroscopy results of the irradiated lines before and after the etching process. The peak at $520 \mathrm{~cm}^{-1}$ which is corresponding of crystalline silicon shifted to a lower energy level after an etching but the peak of silicon oxide remained nearly 

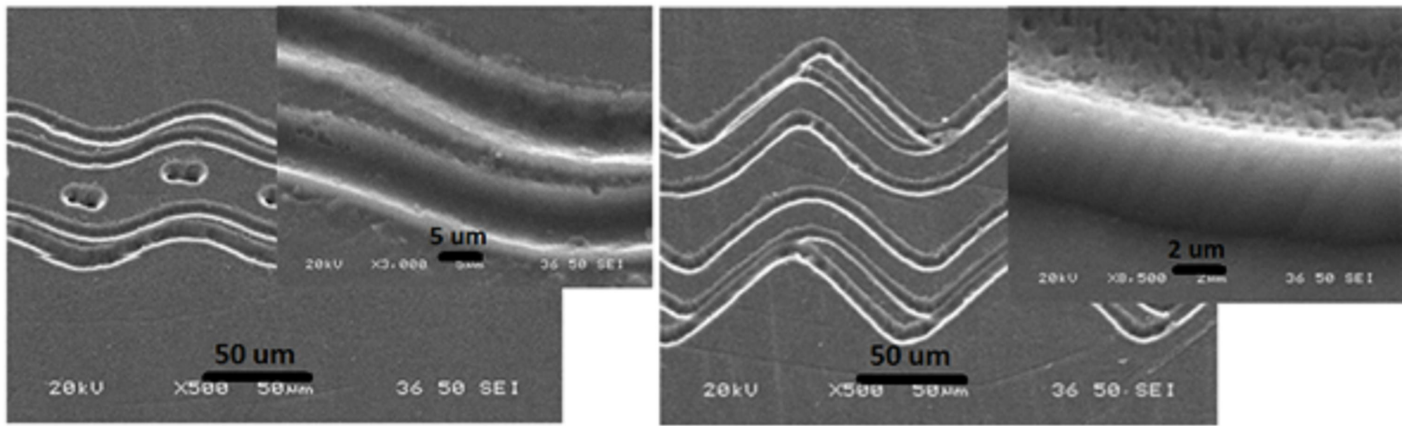

Figure 7.7: SEM images of silicon oxide pattern after etching in $\mathrm{KOH}(30 \%)$ at $65^{\circ} \mathrm{C}$ after 5 min

in the same level of energy supporting the statement that the irradiated area converted to a silicon oxide and acted as an etch stop during the etching process in $\mathrm{KOH}$ [54].

\subsubsection{Maskless lithography using amorphous silicon etch-stop layer}

To study the silicon amorphization, un-doped $<100>$ oriented silicon wafers were used. Prior to laser irradiation, the samples were cleaned with alcohol and ultra pure water. As shown in Figure 7.8, silicon wafers were irradiated with femtosecond pulses with pulse energy well below the damage threshold. Finally, the treated samples were etched in a $\mathrm{KOH}$ solution [15].

Figure 7.9 shows morphology of irradiated samples during the etching process with alkaline etchant $\left(\mathrm{KOH} 30 \%\right.$, temperature: $\left.80^{\circ} \mathrm{C}\right)$. After etching, it was observed that the nonirradiated area and the central region of the laser irradiated zone were etched by alkaline etchant, whereas, the annulus around the central region of the laser spot was not etched. Consequently, a ring feature stood out on the silicon substrate, suggesting that the amorphous layer was resistant to $\mathrm{KOH}$. This etch ratio value agrees well with the result of the etch 


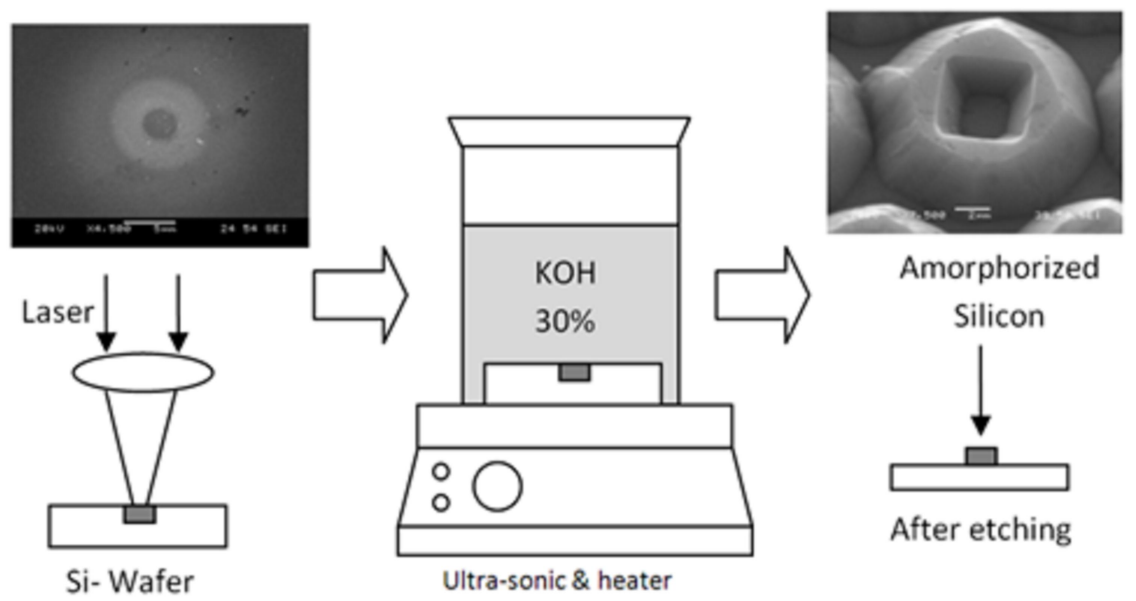

Figure 7.8: Process of experiment

rate of amorphous silicon reported by Kawasegi et al. [27, 29,94]. According to their reports, the etch rate of amorphous silicon generated by magnetron spattering is about 30 times lower than the etch rate of crystalline silicon $<100>$.

Because of the Gaussian distribution of the beam, the laser fluence at the center region was above the surface damage threshold; therefore, ablation occurred in this area. It was observed that after etching, the center zone was removed by the etchant. Thus, the central zone was not in the amorphous state. However, in the surrounding annulus area, the amorphous phase was formed on crystalline silicon, which acted as an etch stop during the etching process.

In order to obtain a smaller circular dot instead of a donut feature, the laser beam should be accurately controlled such that central peak of the Gaussian beam intensity does not exceed the surface damage threshold. Figure 7.10 shows a SEM image of the circular feature obtained after wet etching. 


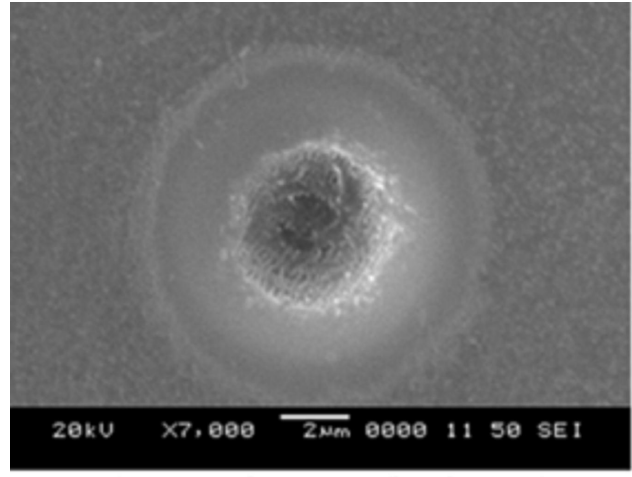

a) During etching process (less than $1 \mathrm{~min}$ )

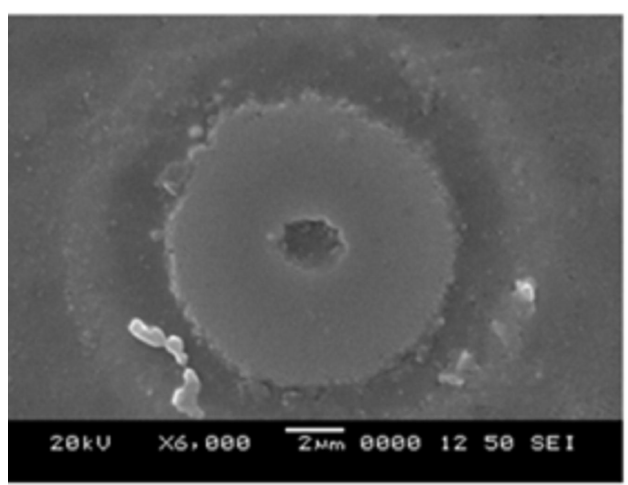

c) During etching process (between $2 \sim 3 \mathrm{~min}$ )

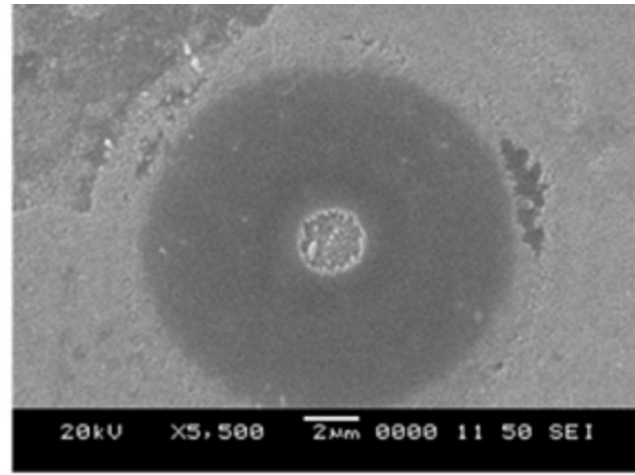

b) During etching process (between $1 \sim 2 \mathrm{~min}$ )

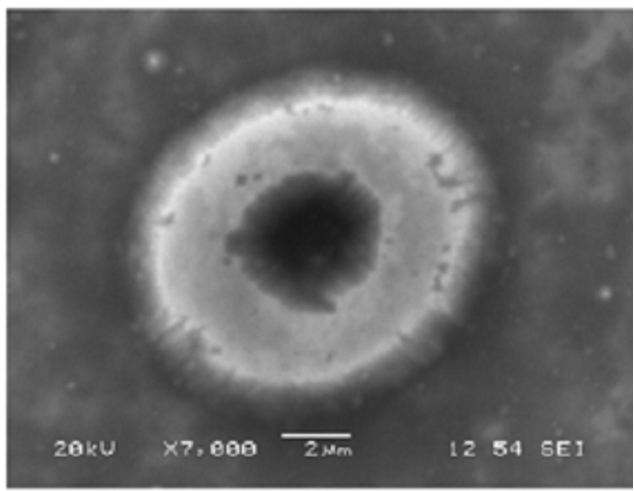

d) During etching process (between $3 \sim 4 \mathrm{~min}$ )

Figure 7.9: SEM images of irradiated samples during etching process

Figure 7.11 presents a line feature with $1.4 \mu m$ thickness and $6 \mu m$ width generated with a scanning laser spot of $0.28 \mathrm{~J} / \mathrm{cm}^{2}$ at $26 \mathrm{MHz}$. As shown in Figure 7.4, the irradiated zone was not etched; however, the non-irradiated area was dissolved during etching and finally, a pyramid-like structure was formed on silicon substrate. The etched (100) planes have a slope with an angle of $54.7^{\circ}$ as expected [95].

As shown in Figure 7.12, the amorphorized layers generated respectively at 8, 13 and $26 \mathrm{M} \mathrm{Hz}$ had a better quality and repeatability at higher pulse frequency (at $26 \mathrm{MHz}$ ). It is due to this fact that with higher pulse frequency at $26 \mathrm{M} \mathrm{Hz}$, the fluctuation in laser power is 

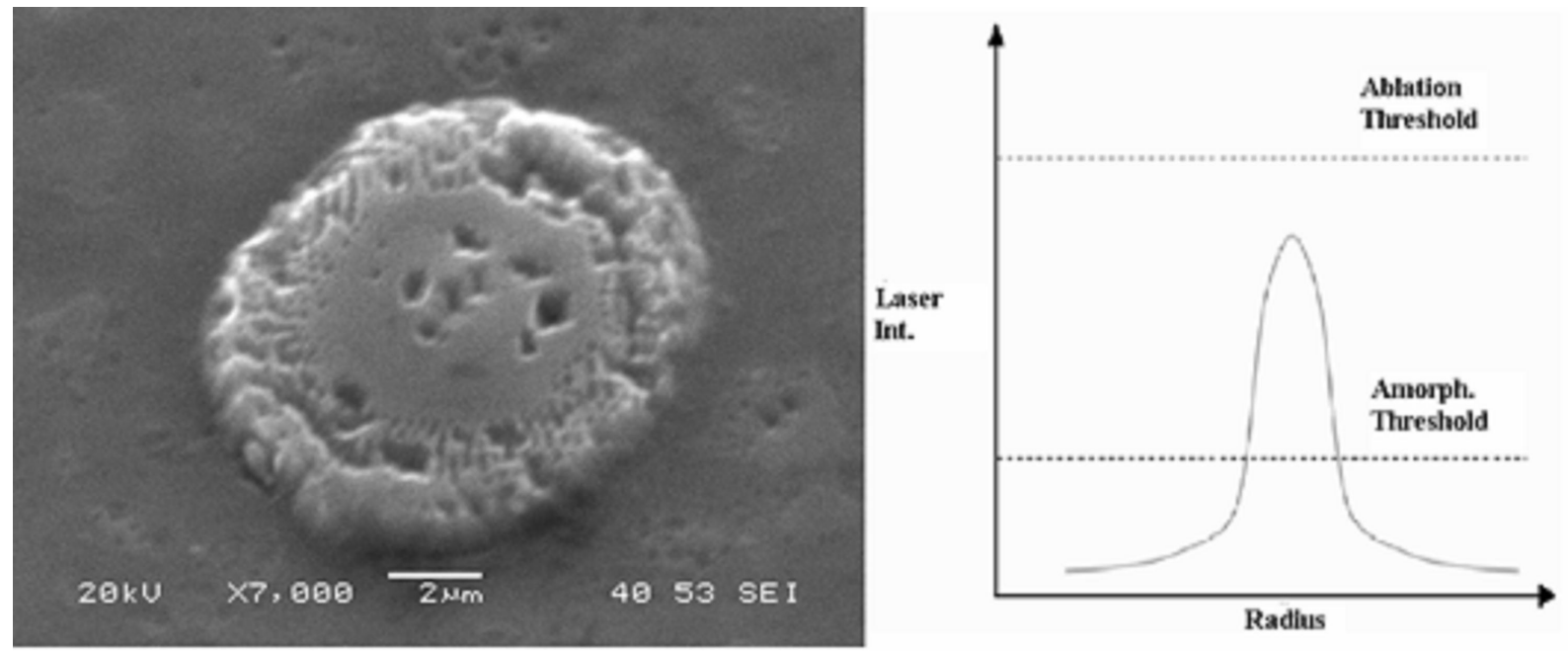

Figure 7.10: SEM image of feature after 3 min etching - solid round at $0.2 \mathrm{~J} / \mathrm{cm}^{2}$
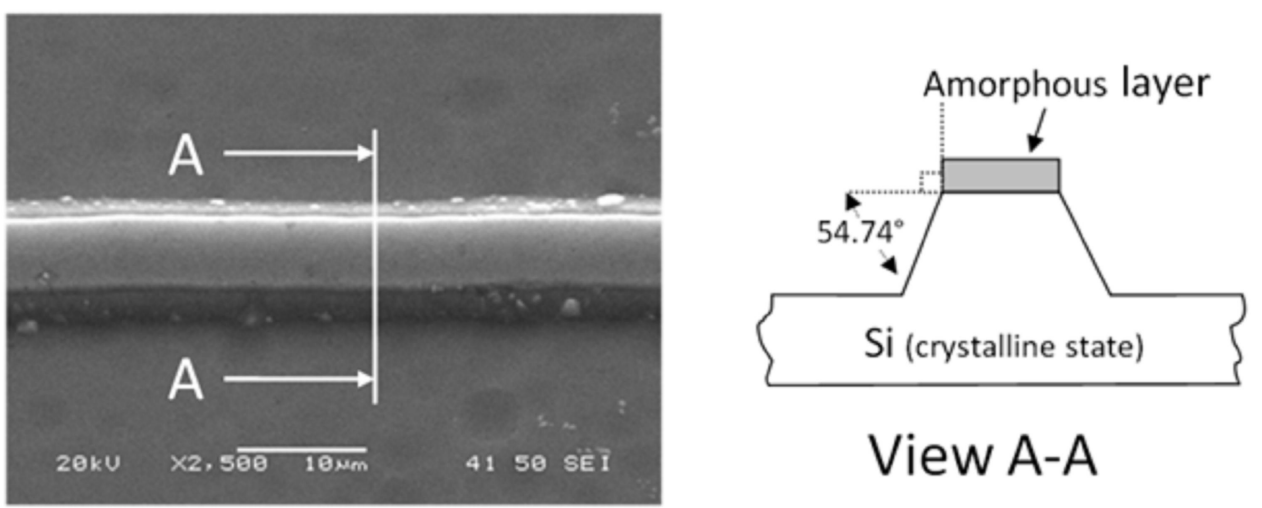

Figure 7.11: SEM image at the incident angle of $35^{\circ}$ of line feature after etching in $\mathrm{KOH}$ (Pulse energy: $\left.0.28 \mathrm{~J} / \mathrm{cm}^{2}\right)$

less in comparison with the power fluctuation at 8 and $13 \mathrm{MHz}$ [15]. In actuality, the silicon amorphization needs a strict parameter control, and the higher power fluctuation in lower pulse frequencies (at 8 and $13 \mathrm{MHz}$ ), may result in ablation or poor quality of the amorphorized layer.

Figure 7.13 presents the smallest lines generated with reduced pulse energy of $0.1 \mathrm{~J} / \mathrm{cm}^{2}$ at a 


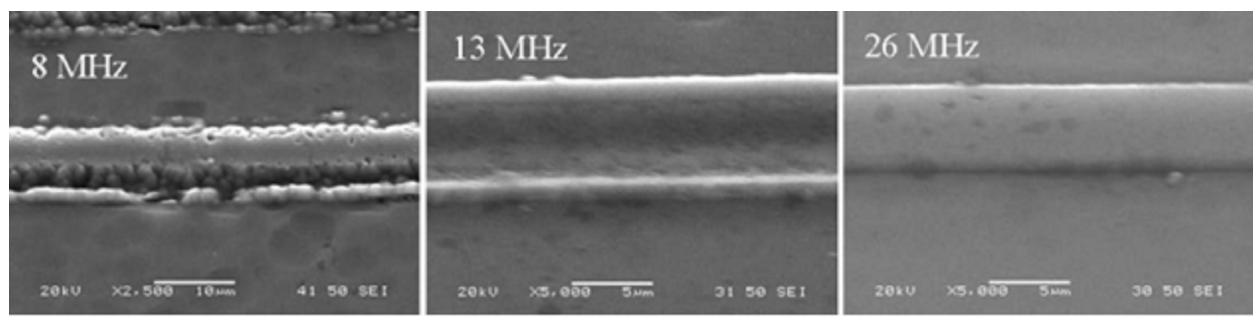

Figure 7.12: SEM images at different pulse repetition rates ](pulse duration: $214 \mathrm{fs}$ )

frequency of $26 \mathrm{M} \mathrm{Hz}$. The line width obtained with the $10 \mu \mathrm{m}$ laser spot is around $150 \mathrm{~nm}$, which is around 1/67 of the laser spot's diameter. Using the same technique, sub-100 nm ablated features generated with a laser spot of $2 \mu \mathrm{m}$ diameter were reported in previous research work [38].

In order to investigate the chemical and structural properties of the generated layer by femtosecond laser pulses on a silicon substrate, Micro-Raman and EDX spectroscopy analyses were performed. EDX analysis of the irradiated zone excluded the possibility of compound formation and a generated layer on the top surface has not been converted to a chemical compound, such as silicon oxide and silicon hydroxide (Figure 7.14).

Raman Spectroscopy analyses of different zones after laser irradiation showed a sharp peak at the wavenumber of $517 \mathrm{~cm}^{-1}$, which is the characteristic peak of crystalline silicon. Furthermore, another peak was observed at the wavenumber of $506.9 \mathrm{~cm}^{-1}$, which is the characteristic of amorphous silicon [62](See Figure 7.15). Therefore, it can be concluded that the irradiated zone was converted from crystalline silicon into amorphous silicon. 


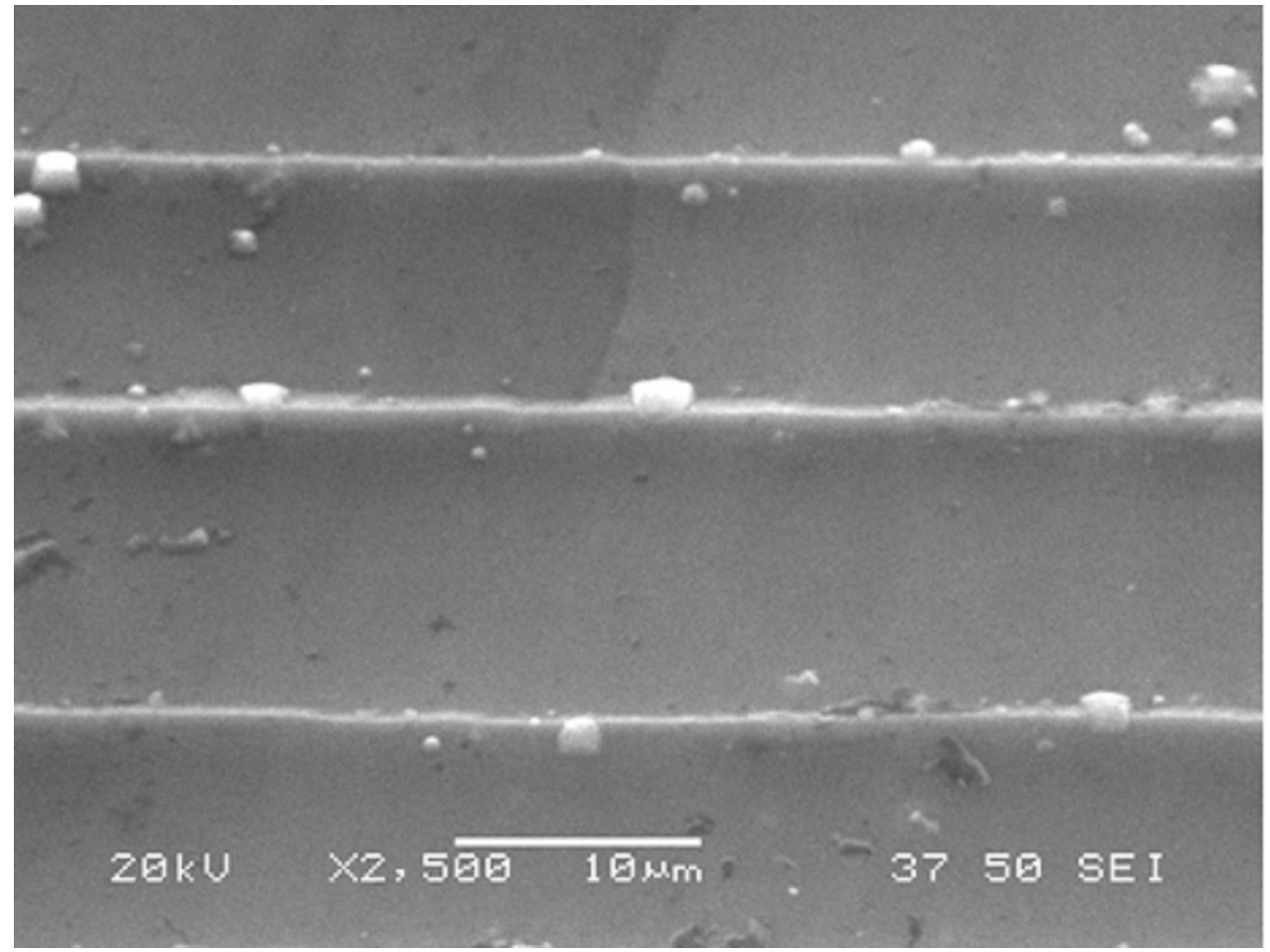

Figure 7.13: $150 \mathrm{~nm}$ line width at pulse energy of $0.1 \mathrm{~J} / \mathrm{cm}^{2}$ (at $26 \mathrm{MHz}$ )

\subsection{Enhancement of the Optical Absorption of Thin- film of Amorphorized Silicon for Photovoltaic En- ergy Conversion}

The use of thin film silicon is one of the most important approaches in photovoltaic devices and solar cell fabrication due mainly to its low material cost and ease of manufacturing $[11-13]$

One major challenge, however, is to improve the light absorption efficiency of thin film silicon 


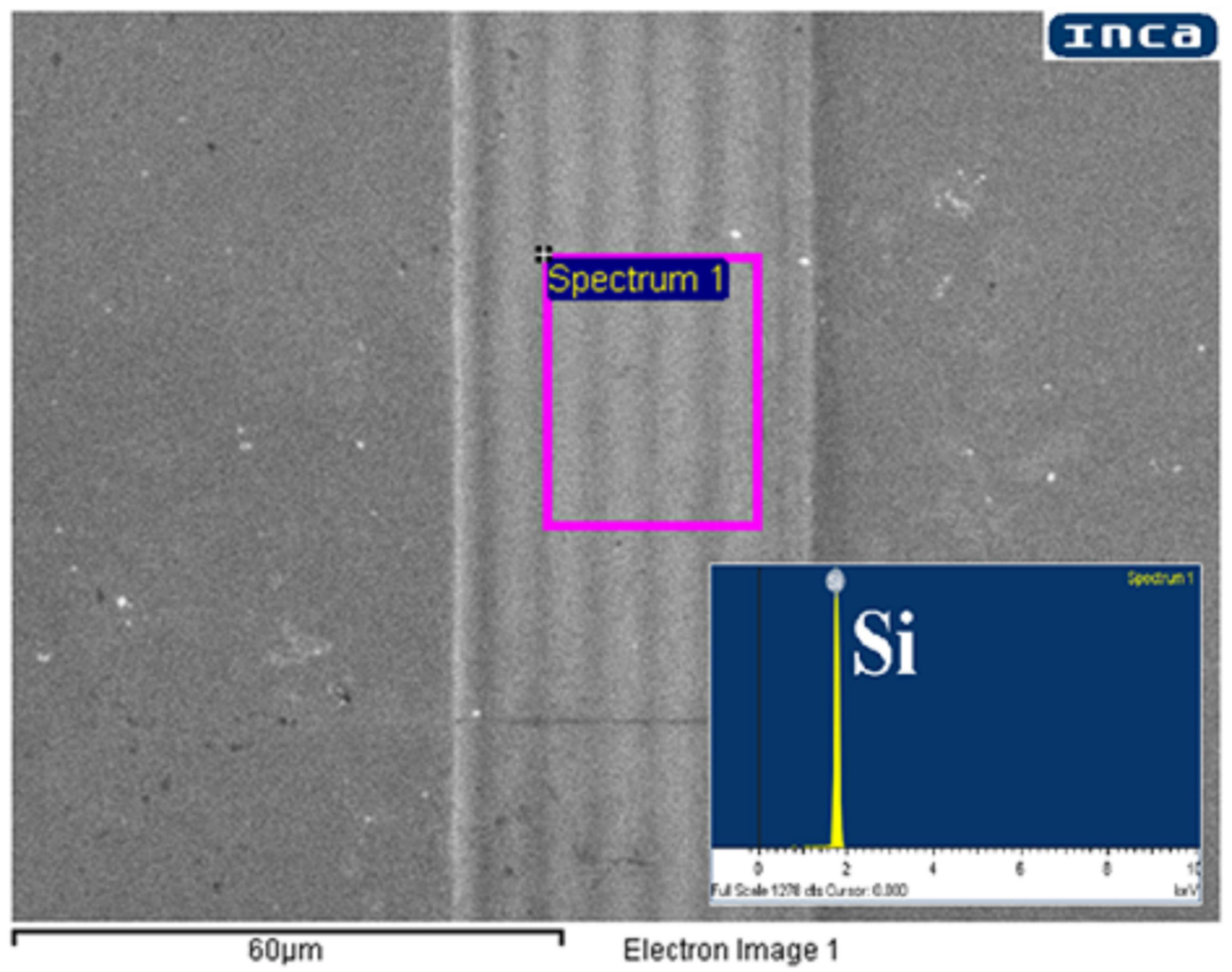

Figure 7.14: EDX analysis of irradiated zone

solar cells. Over the last decades, many approaches have been proposed by researchers to modify the optical properties (reducing reflection and improving light trapping) of thin film silicon solar cells $[96,97]$. These approaches are usually achieved through new solar cell structures and fabrication techniques that improve light trapping and energy conversion capability [97-100]. These new structures depend on thick and thin layers of silicon in different phases and compounds such as amorphous silicon (a-Si), hydrogenated amorphous silicon (a-Si:H), micro and nanocrystalline silicon (m/nc-Si or m/nc-Si: H), silicon oxide and other Thin-Film Silicon (TF-Si) accomplished via CVD, Liquid Phase Epitaxy (LPE) 


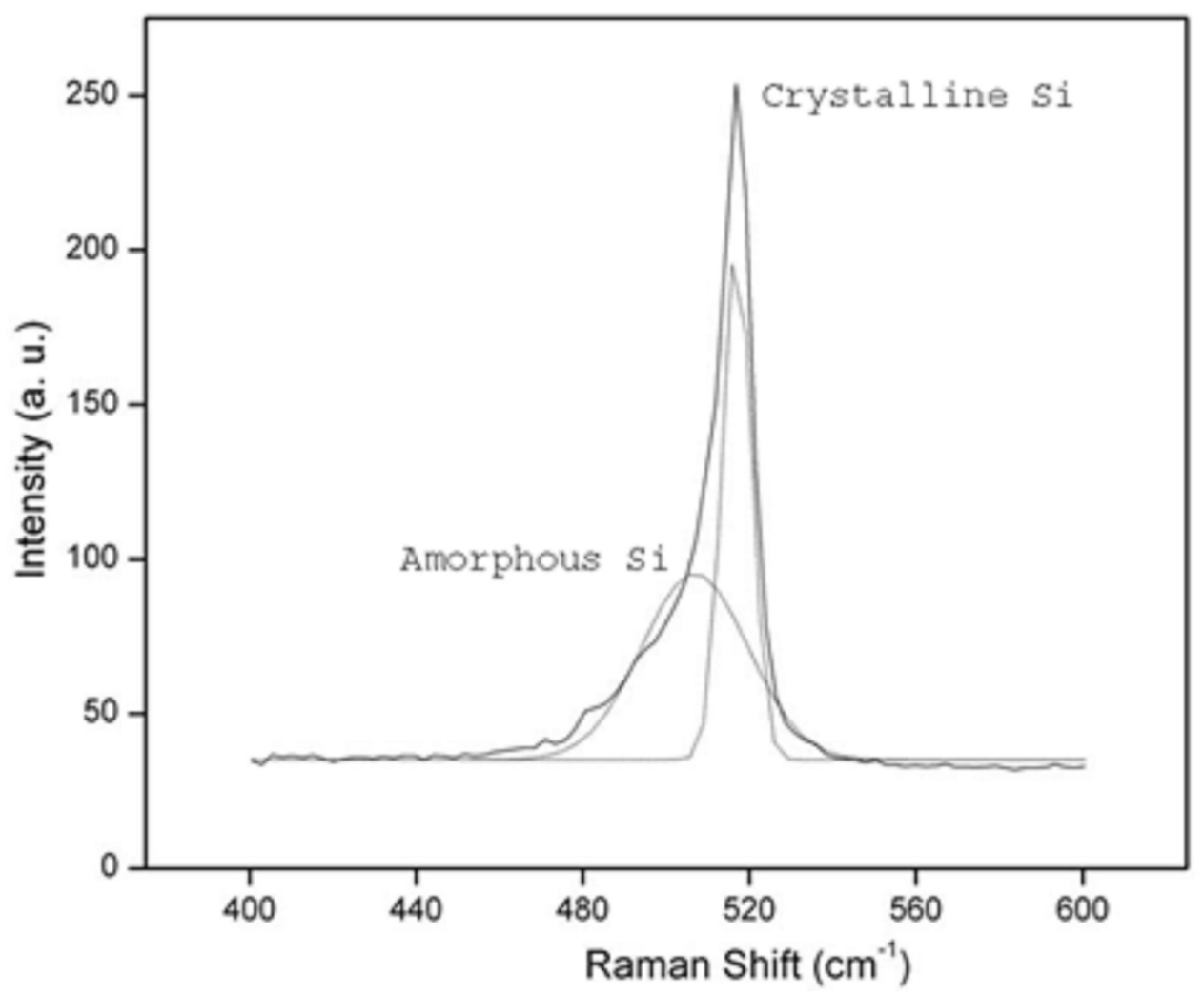

Figure 7.15: Raman spectroscopy graph

or wet and dry thermal oxidation $[11,13,101,102]$. By using some modified techniques such as replacing chemical vapour deposition with PECVD or dry oxidation with wet oxidation, process temperatures can be decreased in order to reduce both processing time and silicon wafer damage [103-105]. Although, these techniques have some advantages, they still suffer from some limitations such as surface damage, process time and the high cost of fabrication. To have a viable technology based on thin-film solar cells it is important to realize a lowcost and high throughput technique compared to the previous techniques as well as to demonstrate a suitable technique for rapid prototyping and custom-scale manufacturing in solar cell fabrication [49]. 
In this chapter, a new method for single-step fabrication of a thin film of amorphous silicon (a-Si) on silicon substrate induced by megahertz frequency laser pulses under ambient conditions was demonstrated. Using high repetition laser pulses with the pulse duration in the range of 200 to 3500 femtosecond enables us to control the laser fluence in the amorphization range of silicon, and finally a thin film of amorphorized silicon of predetermined design would be generated on the silicon substrate. In comparison with previous solar cell fabrication methods based on thin film amorphous silicon, the proposed method is single step and both processing time and wafer damage are significantly reduced. In this method laser energy which is injected into the silicon substrate causes the thin layer of crystalline Si-substrate to be converted to a thin film of amorphous silicon. In other words, this technique does not need to have additive materials, and the amorphorized layer of silicon is grown from a $S i$ wafer substrate [49].

\subsubsection{Results and Discussion}

With carefully controlled laser parameters, amorphorized lines can be created consistently at some points: laser power of 5.2, 5.4,7.2,7.5 and $8 \mathrm{~W}$ at a pulse width of $3500,1400,700,400$ and 200 femtoseconds respectively at a scanning speed of $100 \mathrm{~mm} / \mathrm{s}$ and pulse frequency of $26 \mathrm{MHz}$. Figure 7.16 illustrates parallel amorphorized lines fabricated on silicon substrate at a repetition rate of $26 \mathrm{MHz}$ with the pulse duration of 3500 and 1400 femtosecond (fs) [49].

The irradiated $S i$ samples were coated with gold, before being observed under the SEM, in order to make the surface conductive. In Figure 7.17, EDX results only showed the presence 

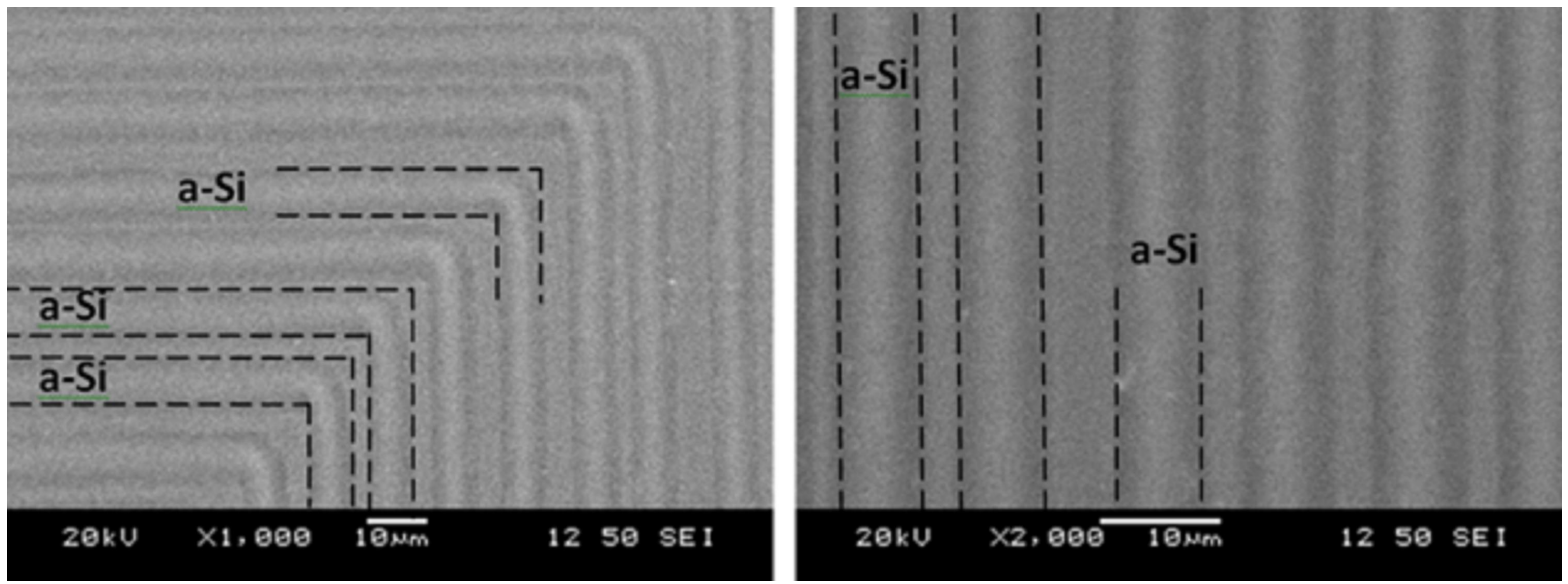

Figure 7.16: SEM images of amorphorized silicon (Frequency: $26 \mathrm{MHz}$, Power: $5.4 \mathrm{~W}$, Scanning speed: $50 \mathrm{~mm} / \mathrm{s}$, Pulse duration: $3500 \mathrm{fs}$ (left), $1400 \mathrm{fs}$ (right))

of silicon $(\mathrm{Si})$ and gold $(\mathrm{Au})$ in irradiated lines which excluded the possibility of compound formation since no trace of oxygen or other materials was observed.

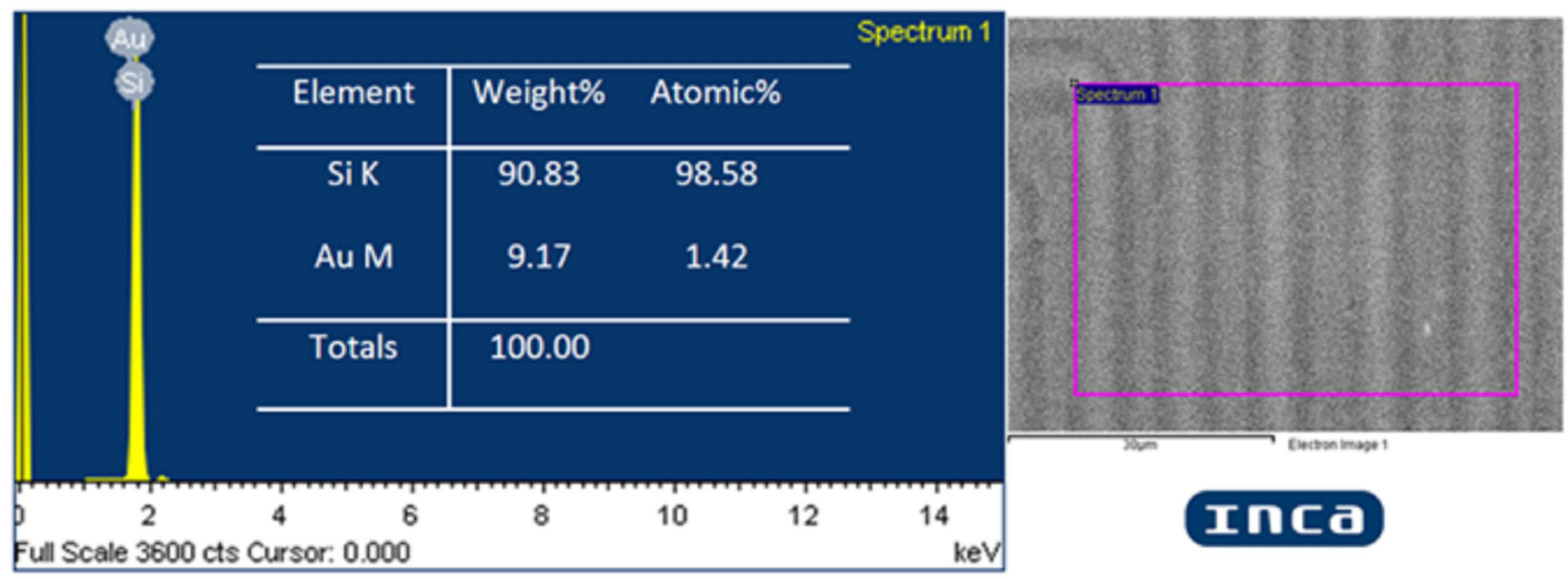

Figure 7.17: EDX results of irradiated area (samples were coated with gold for better spectroscopy results)

In an effort to verify the crystal properties of the irradiated lines which are believed to be made of amorphous silicon, a micro-Raman spectroscopic analysis was conducted. Back 
scattering micro-Raman analysis was performed at ambient conditions using a $532 \mathrm{~nm}$ line of an Ar ion laser source. The Raman spectroscopic measurements on the processed samples showed (Figure 7.18) a sharp peak at the wavenumber of $519 \mathrm{~cm}^{-1}$, which is the characteristic peak of crystalline silicon. Moreover, a secondary peak was observed at the wavenumber of $480 \mathrm{~cm}^{-1}$ indicating the existence of amorphorized silicon in the irradiated area [106]. Therefore, it can be concluded that the irradiated area was converted from crystalline silicon to amorphorized silicon.
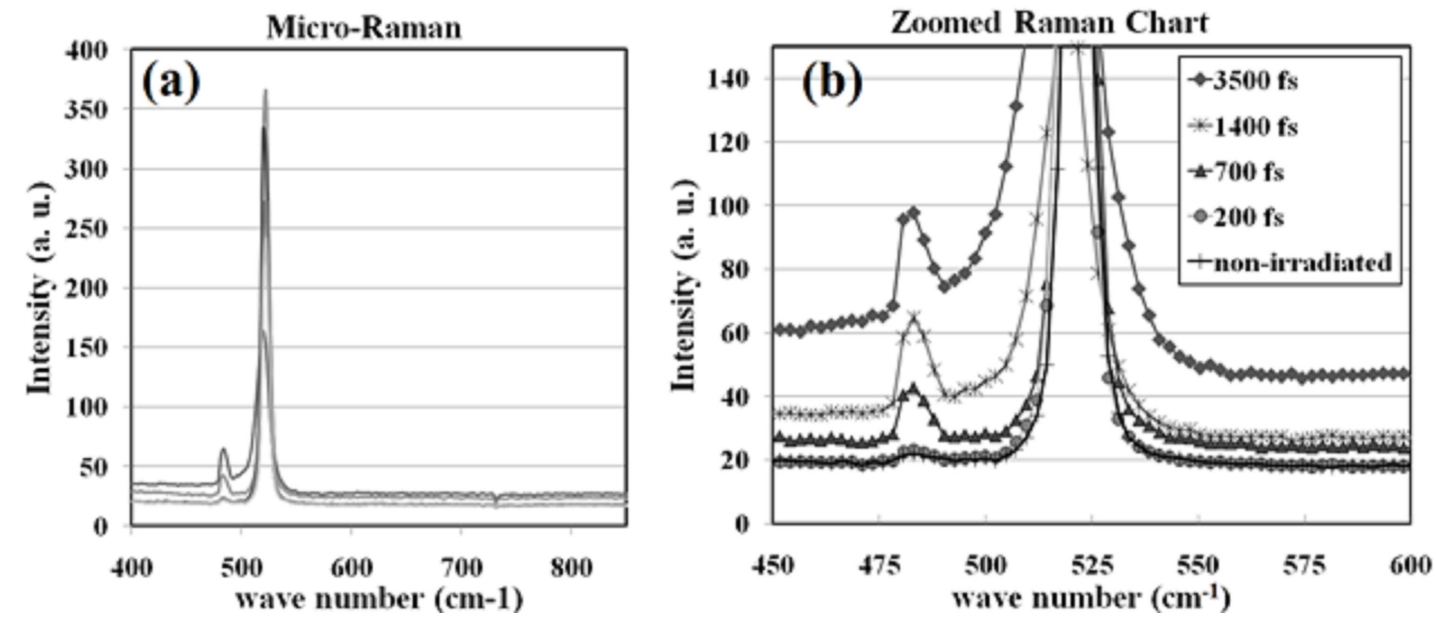

Figure 7.18: Mico-Raman spectroscopy results of amorphorized silicon with various pulse durartion (200 to $3500 \mathrm{fs})$

The detailed (zoomed) Raman chart in Figure 7.18, shows the peak of amorphization shifts to the higher level of intensity (at the same Raman test parameters) by increasing the pulse duration, which means the concentration of amorphorized silicon on the irradiated area increased by increasing pulse duration. The following calculations illustrate how increasing the pulse duration in the experiment corresponds to an increase in the temperature distribution and the amount of energy injected into the target, which leads to a larger amorphorized 
area at a longer pulse duration [48].

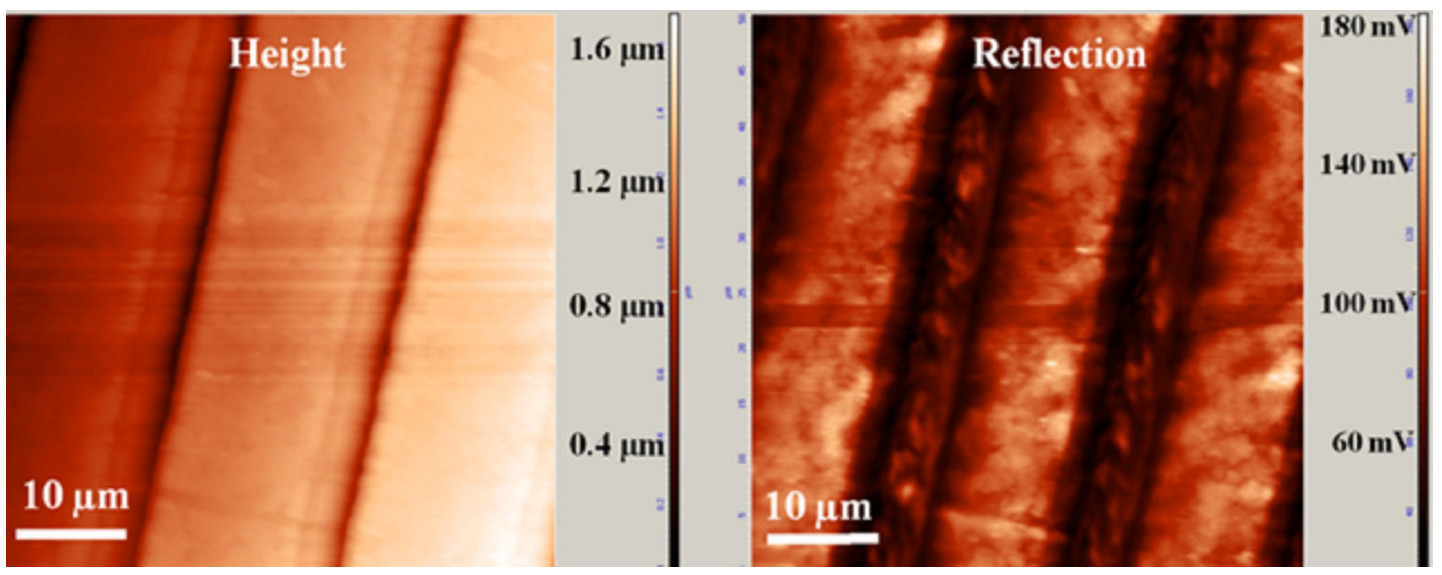

Figure 7.19: Scanning near-field optical microscopy of irradiated samples (Left: surface morphology, Right: light absorption analysis)

Figure 7.19 shows topographic and corresponding reflection images of the amorphorized lines induced by laser pulses at $26 \mathrm{MHz}$ repetition and pulse duration of 3500 femtoseconds using Scanning Near-field Optical Microscopy (SNOM). The SNOM images of the scanned area on the sample exhibit a rich light-absorption in the irradiated (amorphorized) area. These results provide a proof of concept for this technique that can be used to improve the light absorption of solar cells based on thin-film amorphorized silicon [49].

In order to investigate the optical properties of irradiated samples, the reflection spectrum was measured using the $U S B 2000+R A D$ spectroradiometer (Ocean Optics, Dunedin, Florida, USA). The light reflection was measured for wavelengths in the range of $550-1000 \mathrm{~nm}$, with $1 \mathrm{~nm}$ increments. The spectrometer provides a resolution of $0.35 \mathrm{~nm}$ and captures and stores a full spectrum into memory every millisecond. As shown in Figure 7.20, the reflection of the amorphorized silicon surfaces using laser irradiation at wavelengths of $500-1000 \mathrm{~nm}$ is decreased when compared to the untreated surfaces. Furthermore, it was found that by 

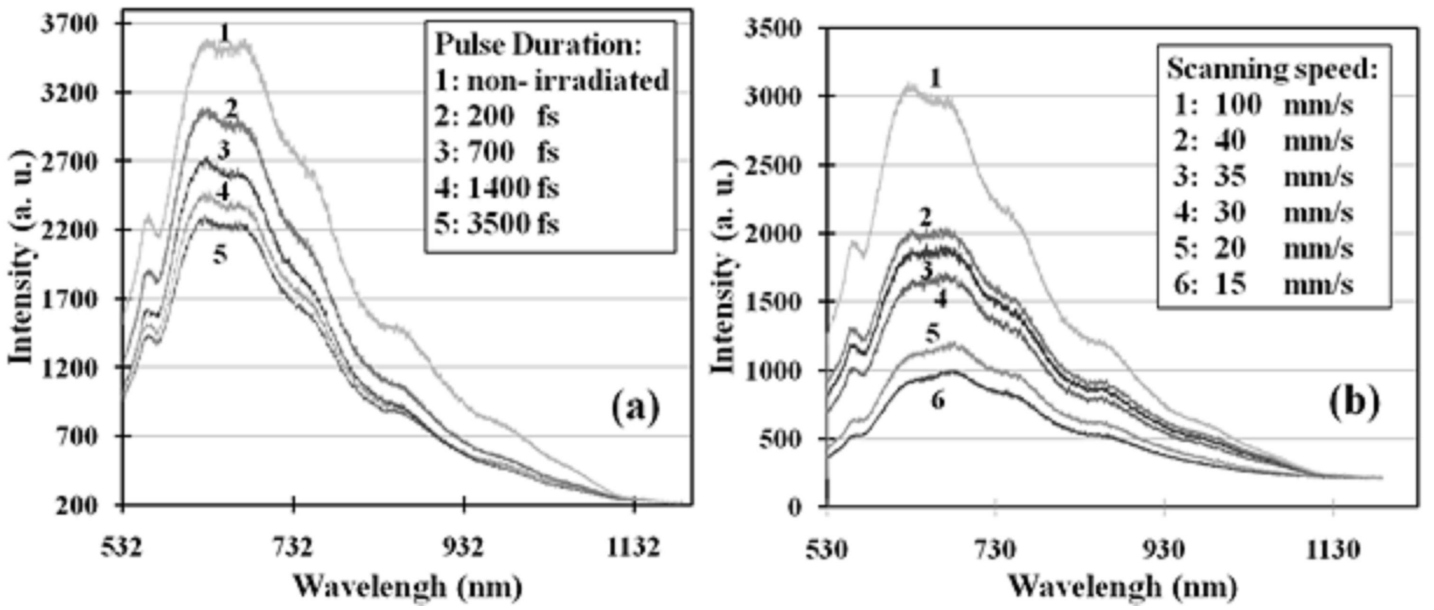

Figure 7.20: Light spectroscopy of amorphorized area induced by laser pulses with (a) different pulse duration and (b) scanning speed

increasing the pulse duration from 200 to 3500 femtosecond the light absorption of the irradiated area in the visible region increases approximately up to $40 \%$ (Figure 7.20 (a)). It is due to this fact that the amorphorized area (heat affected area) becomes larger when laser pulses with longer pulse duration are applied to the silicon substrate (See detailed (zoomed) Raman chart in Figure 7.18).

Generally, amorphous structure has a higher absorption rate of light in comparison with the crystalline structure of the same materials; as reported by researchers, amorphorized silicon has a higher band-gap than silicon in a crystalline state [107]; thus, amorphorized silicon can absorb the visible part of a solar spectrum more effectively than the infrared portion of the spectrum.

After being irradiated by laser pulses with variable scanning speed, the irradiated samples were analyzed using a light spectrometer. Figure 7.19(b) shows that the light-reflection of the amorphorized zones decreased with the various irradiation scanning speeds. The effective 
number of pulses, $N_{\text {eff }}$, can be calculated by $N_{\text {eff }}=\sqrt{\pi / 2} \omega_{0} f / V$, where, $\omega_{0}$ is the machining spot radius, $f$ is the laser repetition rate and $\hat{\mathrm{I}}^{1 / 2}$ is the galvoscanning speed. Corresponding to $15,20,30,35,40$ and $100 \mathrm{~mm} / \mathrm{s}$, the numbers of pulses are 11405, 8554, 5700, 4890, 4277and1710, respectively. In fact, the increase in the pulse number results in the increase of the volume of amorphorized area which leads to improved light absorption. In fact, as the incident pulse number increases, more energy was deposited; therefore, the amorphorized zone (heat affected area) would become larger [72].

\begin{tabular}{|c|l|l|l|l|l|l|l|}
\hline No. & $\begin{array}{l}\text { Pulse } \\
\text { width } \\
(\mathrm{fs})\end{array}$ & $\begin{array}{l}\text { Power } \\
(\mathrm{W})\end{array}$ & $\begin{array}{l}\text { Non- } \\
\text { dimensional } \\
\text { pulse dura- } \\
\text { tion - }\end{array}$ & $\begin{array}{l}\text { Non- } \\
\text { dimensional } \\
\text { power - Q }\end{array}$ & $\begin{array}{l}\text { Non- } \\
\text { dimensional } \\
\text { temperat- } \\
\text { ure }-\Theta\end{array}$ & $\begin{array}{l}\text { Raman } \\
\text { peak of } \\
\text { a-Si at } \\
480 \mathrm{~cm}^{-1} \\
\text { a.u. })\end{array}$ & $\begin{array}{l}\text { Increase } \\
\text { in light } \\
\text { absorp- } \\
\text { tion at } \\
630 ~ \\
\text { nm } \\
\text { a.u. })\end{array}$ \\
\hline 1 & 200 & 8 & $2.514 \times 10^{-6}$ & 1.2192 & 0.06250 & 23.125 & 504 \\
\hline 2 & 400 & 7.5 & $5.030 \times 10^{-6}$ & 1.1430 & 0.08288 & - & 867 \\
\hline 3 & 700 & 7.2 & $8.802 \times 10^{-6}$ & 1.0973 & 0.10526 & 42.675 & 927 \\
\hline 4 & 1400 & 5.4 & $17.604 \times 10^{-6}$ & 0.8230 & 0.11165 & 64.75 & 1162 \\
\hline 5 & 3500 & 5.2 & $44.001 \times 10^{-6}$ & 0.7930 & 0.17008 & 97.8 & 1286 \\
\hline
\end{tabular}

Table 7.1: Analytical and experimental results of the amorphorized silicon wafers

Table 7.1 shows the different results of Raman peaks of a-Si $\left(\right.$ at $\left.480 \mathrm{~cm}^{-1}\right)$, an increase in light absorption at $630 \mathrm{~nm}$ and non-dimensional temperatures for different values of the experiments.

The analytical/experimental results of the experiment for different parameters such as nondimensional temperature, micro-Raman peaks of amorphorized silicon and light absorption of irradiated samples for different pulse widths and laser power levels are shown in Figure 7.21. The computed results in Figure 7.21(a) show that by increasing the pulse duration 

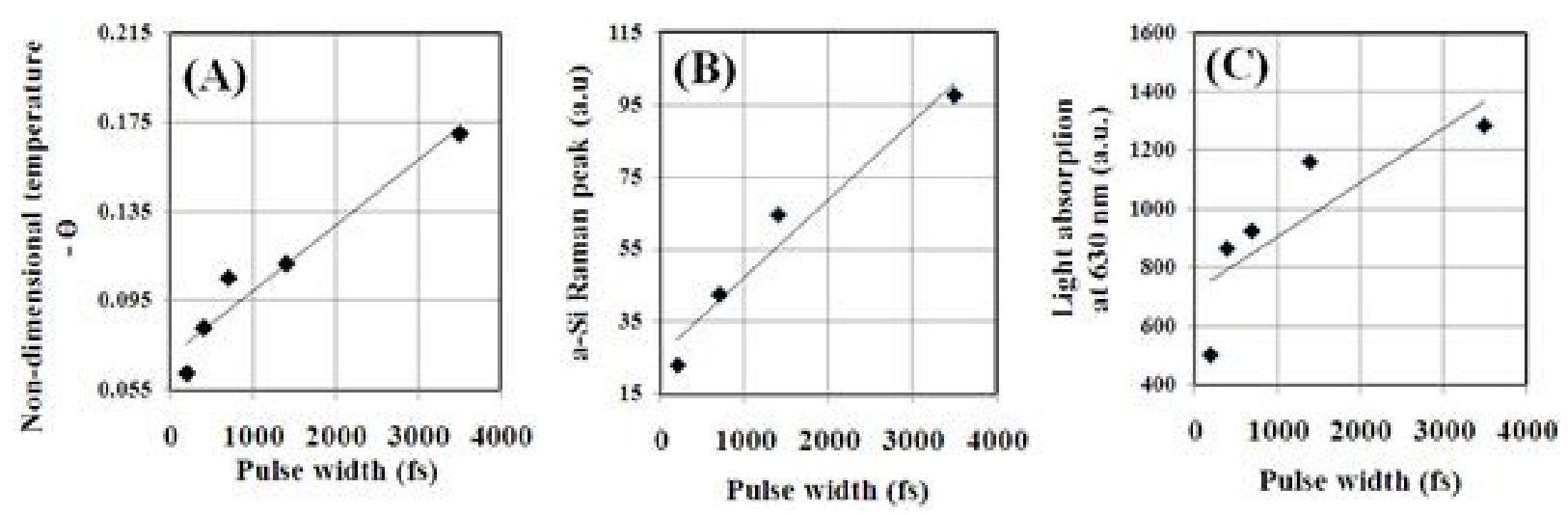

Figure 7.21: Analytical/experimental graphs of: a) non-dimensional surface temperature, b) raman peak of amorphous silicon, c) light absorption at $630 \mathrm{~nm}$

(and decreasing the laser power at the same time) the surface temperature of the irradiated area increased, which means that more energy was injected into the $S i$-substrate; thus, the HAZ, or the volume where the temperature reaches the amorphization temperature, became wider; it resulted in an increase of the amount of silicon converted from a crystalline to amorphorized state as shown in the micro-Raman spectroscopy for the amorphorized silicon peak at $480 \mathrm{~cm}^{-1}$ (Figure 7.21(b)) Moreover, it was found that the light-absorption of amorphorized sample improved by irradiation with longer laser pulses. Although the calculated results are not actual parameters (nondimensional) and they may contain errors due to the assumptions, the tendency of the surface temperature, amorphization rate and improvement in light absorption in relation to pulse duration is quite clear and this can be used explain the phenomenon observed in experimental results [49]. 


\subsection{Summary}

In this chapter, a new method for direct-write maskless lithography, using a combination of femtosecond laser oxidation of silicon and alkaline chemical etching was proposed. The formed oxidized pattern acted as an etch stop in an alkaline etchant, and micro features were generated on a silicon substrate after the etching process. Using megahertz frequency $(\mathrm{MHz})$ femtosecond pulse laser makes this technique suitable for rapid prototyping and custom-scale manufacturing of MEMS, NEMS, Integrated Circuit (IC) and LOC systems, which demand flexibility in re-designing. This proposed method has a potential of fabricating features in a micro/nano scale by optimizing etching and laser parameters such as employing a laser beam of shorter wavelength, a higher Numerical Aperture (NA) focusing lens and reducing pulse energy $[53,54]$.

In the future, more investigations should be carried out to reduce the feature size in a sub-micro scale by optimizing laser parameters and the etching process [54].

Furthermore, a new method for single-step fabrication of a thin film of amorphorized silicon ( $a$-Si $)$ on silicon substrate induced by high repetition femtosecond laser pulses under ambient conditions was demonstrated. Using high repetition laser pulses with the different pulse durations in the range of $200-3500 \mathrm{fs}$ enabled us to control the laser fluence in the amorphization range and finally a thin film of amorphorized silicon could be fabricated

on the Si-substrate. In comparison with previous methods proposed for a-Si thin-film solar cells, the proposed method is single step; there is no need for additive materials and finally, processing time and wafer damage are considerably reduced, which leads to promising 
solutions for thin-film silicon solar cell fabrication [49]. 


\section{Chapter 8}

\section{Summary and Future Research}

\subsection{Summary}

I

$\mathrm{N}$ this thesis, a new method for direct micro/nano amorphization/oxidation of silicon using femtosecond laser irradiation, was demonstrated. The applications of this method in maskless lithography and solar cell fabrication were explored. A systematic theoretical and experimental study of the influence of the laser parameters on the quality of the amorphorized/oxidized area and the size of the features fabricated through amorphization/oxidation has been studied.

This research aimed to investigate the feasibility of using megahertz femtosecond laser pulses for direct amorphization of silicon on silicon substrates. A thin layer of amorphous silicon of predetermined pattern was first generated by irradiation with a femtosecond laser of megahertz pulse frequency (the average laser fluence: $0.15 \sim 0.35 \mathrm{~J} / \mathrm{cm}^{2}$ at repetition rates 
of 13 and $26 \mathrm{MHz}$, dwell time of $0.1 \mathrm{~ms}$, and scanning speed of line features in the range of $100-150 \mathrm{~mm} / \mathrm{s}$ ). The following $\mathrm{KOH}$ etching revealed that the amorphous silicon layer acted as an etch stop. Linewidths less than 1/67 of the focused spot size was demonstrated and hence the proposed method has the potential of producing submicron and nano-scale features by employing a laser beam of shorter wavelength and a high NA focusing lens.

The method for direct patterning of silicon oxide on a silicon substrate by irradiation with a femtosecond laser of megahertz pulse frequency under ambient condition was presented. Embossed lines of silicon oxide with around $3 \sim 4 \mu \mathrm{m}$ width and less than $100 \mathrm{~nm}$ height were formed by controlling the parameters such as laser pulse power and frequency rate $\left(1 \mathrm{~W}\left(9 \mathrm{~J} / \mathrm{cm}^{2}\right)\right.$, at $13 \mathrm{MHz}$ and $0.35 \mathrm{~W}\left(1.65 \mathrm{~J} / \mathrm{cm}^{2}\right)$, at 26 megahertz with a scanning speed of $500 / \mathrm{mm} / \mathrm{s}$ ). It was found that the direct oxidation with femtosecond laser requires stringent parameter controls. Even a slight deviation in laser parameters, for example, fluctuation in laser power, will result in ablation. Therefore, higher pulse frequency is preferred for oxidation since at the same laser power the pulse energy fluctuation is lower at higher pulse frequency. Furthermore, the absolute power fluctuation is less in lower powers such as $0.35 \mathrm{~W}$ rather than $1 \mathrm{~W}$ and higher. This explains why better repeatability was observed at $26 \mathrm{M} \mathrm{Hz}$ during the experiments. This study indicates that an oscillator, which has higher repetition rate and lower average laser power, is more suitable for this task than the amplified system used for this experiment.

Additionally, this research work aimed to study the effects of laser parameters on direct silicon amorphization. It was found that higher repetition rate of laser pulses gives smooth morphology with better repeatability. Increasing pulse duration and number of pulses were seen to increase the line width. However, increasing the number of pulses does not result 
in ablation of the target area. An analytical model is developed for the calculation of the average surface temperature after n-pulses; it was found that for a constant power and a constant repetition rate, an increase in the pulse number does not correspond to a significant increase in the surface temperature.

Moreover, at the controlled laser power level, the surface temperature will not exceed the melting point of silicon. Therefore, thermal induced damage is not observed during the amorphization. Furthermore, the effect of laser pulse width was investigated by developing an analytical model for the calculation of the non-dimensional surface temperature with various pulse widths; it was found from experimental and analytical results that for a constant power and repetition rate, an increase in the pulse duration corresponds to a significant increase in the surface temperature which results in an increase in the amount of amorphorized material as well as improvement of light absorption.

The feasibility of using this technique as a promising solution for maskless lithography was carried out in this research; the direct oxidation/amorphization of silicon induced by the femtosecond laser is a maskless single-step technique which offers a higher flexibility and reduced processing time. In addition this method allows for large-area patterning (in mm scale) at fast writing speed under ambient condition.

A new method was demonstrated for the single-step fabrication of a thin film of amorphous silicon (a-Si) on silicon substrate induced by megahertz frequency laser pulses under ambient conditions. Using high repetition laser pulses with the pulse duration in the range of 200 to $3500 \mathrm{fs}$ enables us to control the laser fluence in the amorphization range of silicon, and finally a thin film of amorphorized silicon of predetermined design would be generated on 
the silicon substrate. In comparison with previous solar cell fabrication methods based on thin film amorphous silicon, the proposed method is single step, and both processing time and wafer damage are significantly reduced. In this method laser energy, which is injected into the silicon substrate, causes the thin layer of crystalline Si-substrate to be converted to a thin film of amorphous silicon. In other words, this technique does not need to have additive materials, and the amorphorized layer of silicon is grown from a $S i$ wafer substrate.

The main contribution of this research is highlighted as follows:

- Demonstrated a new method for micro/nano scale direct writing of amorphorized/ oxidized silicon on silicon substrates induced by megahertz frequency femtosecond laser pulses; using megahertz femtosecond laser pulses enable us to control the laser fluence below the ablation threshold; thus, a thin-film of amorphorized/oxidized silicon can be generated on silicon substrate.

- Developed a new technique for micro/nano maskless lithography; the proposed technique is a maskless single-step technique which offers a higher flexibility and reduced processing time. In comparison with conventional methods, the proposed technique allows for large-area patterning (in $\mathrm{mm}$ scale) at fast writing speed under ambient conditions.

- Demonstrated a new method for fabrication of thin-film amorphorized silicon for solar cell fabrication; This method is single step and, in comparison with conventional $a-S i$ thin-film solar cell fabrication methods, both processing time and wafer damage are significantly reduced. Moreover, this technique does not require additive materials. 
This is the first time that the concept of direct growing micro/nano scale of amorphorized/oxidized silicon from the surface of a $S i$ substrate is proposed, and verified and developed by experiment. The key features of this method are as follows:

- the processing is fast and single step

- can be used for micro/nano scale patterning

- can be done under ambient conditions

These key features make this technique suitable for rapid prototyping and low-volume manufacturing, and also can lead to improvements in efficiency, cost and durability.

\subsection{Further Research}

The following would be the directions for future work in applying and enhancing the current work with more accuracy.

- Further study of physics of amorphization/oxidation of silicon induced by laser pulses at various pulse wavelengths;

- Study different polarizations of irradiation on silicon substrates with pulse energy well below ablation threshold and the effect of different polarization on the thickness of the generated amorphous/oxide layer of Si substrate;

- Study double pulse laser beam irradiation (530 and $1040 \mathrm{~nm}$ ) and its effect on the process of oxidation or amorphization; 
- Investigate the feasibility of using the generated features (via lithography, as mentioned previously) as a stamp for soft lithography and fabrication of micro and nano channels for various applications in LOC and biomedical systems;

- Increase the light absorption and photovoltaic conversion efficiency of the current method for a-Si thin-film solar cell fabrication, by generation of weblike fibrous nanostructures consisting of agglomerated silicon nanoparticles on the generated amorphorized silicon layer. This combined nanofiber and thin=film generation technique can result in a significant drop in reflectance of the irradiated surfaces in the visible range. This is attributed to the increase in surface area of the laser irradiated surfaces which in turn increases the absorption of incident light. 


\section{Appendices}




\section{Appendix A}

\section{Properties of Silicon at Room}

\section{Temperature}

\begin{tabular}{|c|c|c|}
\hline \multicolumn{3}{|c|}{ Structural and Mechanical } \\
\hline Atomic weight & 28.09 & \\
\hline Atoms, total $\left(\mathrm{cm}^{-3}\right)$ & $4.995 \times 10^{22}$ & \\
\hline Crystalline structure & Diamond $(\mathrm{FCC})$ & \\
\hline Lattice constant $(\AA)$ & 5.43 & \\
\hline Density $\left(\mathrm{g} / \mathrm{m}^{3}\right)$ & 2.33 & \\
\hline & $(100) 6.78 \times 10^{14}$ & \\
\hline Density of surface atoms $\left(\mathrm{cm}^{-2}\right)$ & $(110) 9.59 \times 10^{14}$ & \\
& $(111) 7.83 \times 10^{14}$ & \\
\hline Young's modulus $(\mathrm{GPa})$ & 190 & \\
\hline
\end{tabular}




\begin{tabular}{|c|c|c|}
\hline Yield strength & 7 & (111) crystalline orientation \\
\hline Fracture strain & $4 \%$ & \\
\hline Poisson ratio, $v$ & 0.27 & \\
\hline Knoop hardness $\left(\mathrm{kg} / \mathrm{mm}^{2}\right)$ & 850 & \\
\hline \multicolumn{3}{|c|}{ Electrical } \\
\hline Energy gap $(\mathrm{eV})$ & 1.12 & \\
\hline Intrinsic carrier concentration $\left(\mathrm{cm}^{-3}\right)$ & $1.38 \times 10^{10}$ & \\
\hline and resistivity $(\Omega-\mathrm{cm})$ & $2.3 \times 10^{5}$ & \\
\hline Dielectric constant & 11.8 & \\
\hline Intrinsic Debye length (nm) & 24 & \\
\hline Mobility (drift) $\left(\mathrm{cm}^{2} / V s\right)$ & $\begin{array}{c}1500 \text { (electrons) } \\
475 \text { (holes) }\end{array}$ & \\
\hline Temp. coeff. Of resistivity $\left(K^{-1}\right)$ & 0.0017 & \\
\hline \multicolumn{3}{|c|}{ Thermal } \\
\hline Coeff. Of thermal expansion $\left({ }^{\circ} \mathrm{C}^{-1}\right)$ & $2.6 \times 10^{-6}$ & \\
\hline Melting point $\left({ }^{\circ} \mathrm{C}\right)$ & 1414 & \\
\hline Specific heat $(\mathrm{j} / \mathrm{kgK})$ & 700 & \\
\hline Thermal conductivity $(\mathrm{W} / \mathrm{m} \mathrm{K})$ & 150 & \\
\hline Thermal diffusivity $\left(\mathrm{cm}^{2} / \mathrm{s}\right)$ & 0.8 & \\
\hline \multicolumn{3}{|c|}{ Optical } \\
\hline Index of refraction & $\begin{array}{l}3.42 \\
3.48\end{array}$ & $\begin{array}{c}\lambda=632 \mathrm{~nm} \\
\lambda=1550 \mathrm{~nm}\end{array}$ \\
\hline
\end{tabular}




\begin{tabular}{|c|c|c|}
\hline Energy gap wavelength $(\mu \mathrm{m})$ & 1.1 & Transparent at larger wavelength \\
\hline \multirow{3}{*}{ Absorption } & $>10^{6} \mathrm{~cm}^{-1}$ & $\lambda=200-360 \mathrm{~nm}$ \\
& $10^{5} \mathrm{~cm}^{-1}$ & $\lambda=420 \mathrm{~nm}$ \\
$10^{4} \mathrm{~cm}^{-1}$ & $\lambda=550 \mathrm{~nm}$ \\
$10^{3} \mathrm{~cm}^{-1}$ & $\lambda=800 \mathrm{~nm}$ \\
& $<0.01 \mathrm{~cm}^{-1}$ & $\lambda=1500 \mathrm{~nm}$ \\
\hline
\end{tabular}

Table A.1: Properties of silicon at room temperature [10] 


\section{Appendix B}

\section{List of Publications}

\section{Refereed Journals}

1. Amirkianoosh Kiani, K. Venkatakrishnan, B. Tan, V. Venkataramanan, Maskless lithography using silicon oxide etch-stop layer induced by megahertz repetition femtosecond laser pulses, Optics Express 19, 10834 (2011); (impact factor: 3.7);

2. Amirkianoosh Kiani, K. Venkatakrishnan, B. Tan, Enhancement of the optical absorption of thin-film of amorphorized silicon for photovoltaic energy conversion, Solar Energy 85, 1817 (2011); (impact factor: 2.9)

3. Amirkianoosh Kiani, K. Venkatakrishnan, B. Tan, Direct laser patterning of amorphous silicon on Si-Substrate induced by High repetition femtosecond pulses, Journal of Applied Physics 108, (2010) [Selected into November 2010 issue of Virtual Journal of Ultrafast Science]; (impact factor: 2.2)[Number of citation:1]; 
4. Amirkianoosh Kiani, K. Venkatakrishnan, B. Tan, Direct patterning of silicon oxide on Si-substrate induced by femtosecond laser, Optics Express 18, 1872 (2010); (Impact Factor: 3.7) [Number of citation:6];

5. Amirkianoosh Kiani, K. Venkatakrishnan, B. Tan, Micro/nano scale amorphization of silicon by femtosecond laser irradiation, Optics Express 17, 16518 (2009); (Impact Factor: 3.7) [number of citation: 6];

6. Amirkianoosh Kiani, Palneet Singh Waraich, Krishnan Venkatakrishnan, Bo Tan, Synthesis of 3D nanostructured metal alloy of immiscible materials induced by megahertz repetition femtosecond laser pulses, Nanoscale Research Letters (2012) [acceptedto be published], (impact factor: 2.7 )

\section{Refereed Conference Papers}

1. Amirkianoosh Kiani, K. Venkatakrishnan, B. Tan, Optical absorption enhancement of thin-film amorphous silicon induced by femtosecond laser pulses for solar cell fabrication, Nanotechnology 3, 709 (2011);

2. Amirkianoosh Kiani, K. Venkatakrishnan, B. Tan, Maskless Lithography Using Patterned Amorphous Silicon Layer Induced by Femtosecond Laser Irradiation, Nanotechnology 2, 276 (2010)

3. Amirkianoosh Kiani, K. Venkatakrishnan, B. Tan, Direct-write maskless lithography using patterned oxidation of Si-substrate induced by femtosecond laser pulses, SPIE 
Advanced Lithography (2013) [accepted -to be published]

\section{Submitted to Refereed Journals}

1. Amirkianoosh Kiani, K. Venkatakrishnan, B. Tan, Enhancement of the optical absorption of nano sandwich thin-film of amorphorized silicon coated by 3D silicon oxide nanofibrous structures for photovoltaic energy conversion, Progress in Photovoltaics: Research and Applications [submitted -under review] 


\section{References}

[1] D. Bäuerle, Laser processing and chemistry. Springer Verlag, 2011.

[2] D. Lim, Y. Kamotani, B. Cho, J. Mazumder, and S. Takayama, "Fabrication of microfluidic mixers and artificial vasculatures using a high-brightness diode-pumped nd: Yag laser direct write method," Lab Chip, vol. 3, no. 4, pp. 318-323, 2003.

[3] D. Plummer and M. Deal, "Pb griffin silicon vlsi technology," 2000.

[4] Y. Chabal, Fundamental aspects of silicon oxidation. Springer Verlag, 2001, vol. 46.

[5] M. Green, E. Gusev, R. Degraeve, and E. Garfunkel, "Ultrathin $(<4 \mathrm{~nm})$ sio and si$\mathrm{o}-\mathrm{n}$ gate dielectric layers for silicon microelectronics: Understanding the processing, structure, and physical and electrical limits," Journal of Applied Physics, vol. 90, p. 2057, 2001.

[6] G. Aygun, E. Atanassova, A. Alacakir, L. Ozyuzer, and R. Turan, "Oxidation of si surface by a pulsed nd: Yag laser," Journal of Physics D: Applied Physics, vol. 37, p. 1569, 2004. 
[7] A. Grayson, R. Shawgo, A. Johnson, N. Flynn, Y. Li, M. Cima, and R. Langer, "A biomems review: MEMS technology for physiologically integrated devices," Proceedings of the IEEE, vol. 92, no. 1, pp. 6-21, 2004.

[8] G. Saini, R. Gates, M. Asplund, S. Blair, S. Attavar, and M. Linford, "Directing polyallylamine adsorption on microlens array patterned silicon for microarray fabrication," Lab Chip, vol. 9, no. 12, pp. 1789-1796, 2009.

[9] D. Lee, S. Park, H. Yang, K. Chung, T. Yoon, S. Kim, K. Kim, and Y. Kim, "Bulkmicromachined submicroliter-volume pcr chip with very rapid thermal response and low power consumption," Lab Chip, vol. 4, no. 4, pp. 401-407, 2004.

[10] S. Franssila, Introduction to microfabrication. Wiley Online Library, 2004.

[11] K. R Catchpole, M. J McCann, K. J Weber, and A. W Blakers, "A review of thin-film crystalline silicon for solar cell applications. part 2: Foreign substrates," Solar energy materials and solar cells, vol. 68, no. 2, pp. 173-215, 2001.

[12] M. Green, "Crystalline and thin-film silicon solar cells: state of the art and future potential," Solar energy, vol. 74, no. 3, pp. 181-192, 2003.

[13] A. Shah, H. Schade, M. Vanecek, J. Meier, E. Vallat-Sauvain, N. Wyrsch, U. Kroll, C. Droz, and J. Bailat, "Thin-film silicon solar cell technology," Progress in photovoltaics: Research and applications, vol. 12, no. 2-3, pp. 113-142, 2004.

[14] M. Quirk and J. Serda, Semiconductor manufacturing technology. Prentice Hall New Jersey, 2001, vol. 3 . 
[15] A. Kiani, K. Venkatakrishnan, and B. Tan, "Micro/nano scale amorphization of silicon by femtosecond laser irradiation," Optics Express, vol. 17, no. 19, pp. 16518-16526, 2009.

[16] H. Yasuda, S. Arai, J. Kai, Y. Ooae, T. Abe, S. Maruyama, and T. Kiuchi, "Multielectron beam blanking aperture array system synapse-2000," Journal of Vacuum Science 86 Technology B: Microelectronics and Nanometer Structures, vol. 14, no. 6, p. 3813, 1996.

[17] B. Schmidt, L. Bischoff, and J. Teichert, "Writing fib implantation and subsequent anisotropic wet chemical etching for fabrication of 3d structures in silicon," Sensors and Actuators A: Physical, vol. 61, no. 1-3, pp. 369-373, 1997.

[18] J. Dagata, J. Schneir, H. Harary, C. Evans, M. Postek, and J. Bennett, "Modification of hydrogen-passivated silicon by a scanning tunneling microscope operating in air," Applied physics letters, vol. 56, no. 20, pp. 2001-2003, 1990.

[19] S. Minne, H. Soh, P. Flueckiger, and C. Quate, "Fabrication of $0.1 \mu \mathrm{m}$ metal oxide semiconductor field-effect transistors with the atomic force microscope," Applied physics letters, vol. 66, p. 703, 1995.

[20] P. Chapbell, E. Snow, and P. McMarr, "Fabrication of nanometer-scale side-gated silicon field-effect transistors with an atomic-force microwave," Appl. Phys. Lett, vol. 66, no. 11, pp. 1388-1390, 1995.

[21] F. Chien, C. Wu, Y. Chou, T. Chen, S. Gwo, and W. Hsieh, "Nanomachining of (110)- 
oriented silicon by scanning probe lithography and anisotropic wet etching," Applied physics letters, vol. 75, p. 2429, 1999.

[22] Y. Zhang, J. Zhang, G. Luo, X. Zhou, G. Xie, T. Zhu, and Z. Liu, "Fabrication of silicon-based multilevel nanostructures via scanning probe oxidation and anisotropic wet etching," Nanotechnology, vol. 16, p. 422, 2005.

[23] K. Ueno, R. Okada, K. Saiki, and A. Koma, "Nano-scale anodic oxidation on a si (111) surface terminated by bilayer-gase," Surface science, vol. 514, no. 1, pp. 27-32, 2002.

[24] F. Chien, J. Chang, S. Lin, Y. Chou, T. Chen, S. Gwo, T. Chao, and W. Hsieh, "Nanometer-scale conversion of si3n4 to siox," Applied Physics Letters, vol. 76, no. 3, pp. 360-362, 2000.

[25] D. Weinberger, S. Hong, C. Mirkin, B. Wessels, and T. Higgins, "Combinatorial generation and analysis of nanometer-and micrometer-scale silicon features via dip-pen nanolithography and wet chemical etching," Advanced Materials, vol. 12, no. 21, pp. 1600-1603, 2000.

[26] H. Lee, J. Oh, S. Park, K. Park, J. Ha, H. Yoo, and J. Koo, "Nanometer-scale lithography on h-passivated si (100) by atomic force microscope in air," Journal of Vacuum Science 85 Technology A: Vacuum, Surfaces, and Films, vol. 15, no. 3, pp. 1451-1454, 1997.

[27] N. Kawasegi, N. Morita, S. Yamada, N. Takano, T. Oyama, and K. Ashida, "Etch 
stop of silicon surface induced by tribo-nanolithography," Nanotechnology, vol. 16, p. 1411, 2005.

[28] J. Park, N. Kawasegi, N. Morita, and D. Lee, "Tribonanolithography of silicon in aqueous solution based on atomic force microscopy," Applied physics letters, vol. 85, no. 10, pp. 1766-1768, 2004.

[29] J. Park, N. Kawasegi, N. Morita, and D. Lee, "Mechanical approach to nanomachining of silicon using oxide characteristics based on tribo nanolithography (tnl) in koh solution," Journal of manufacturing science and engineering, vol. 126, p. 801, 2004.

[30] R. Willardson and R. Young, Pulsed laser processing of semiconductors. Academic Pr, 1984, vol. 23.

[31] J. Shieh, Z. Chen, B. Dai, Y. Wang, A. Zaitsev, and C. Pan, "Near-infrared femtosecond laser-induced crystallization of amorphous silicon," Applied physics letters, vol. 85, p. 1232, 2004.

[32] J. Jia, M. Li, and C. Thompson, "Amorphization of silicon by femtosecond laser pulses," Applied physics letters, vol. 84, p. 3205, 2004.

[33] B. Chichkov, C. Momma, S. Nolte, F. Von Alvensleben, and A. Tünnermann, "Femtosecond, picosecond and nanosecond laser ablation of solids," Applied Physics A: Materials Science \& Processing, vol. 63, no. 2, pp. 109-115, 1996.

[34] L. De Stefano, I. Rea, M. Arcangela Nigro, F. Della Corte, and I. Rendina, "A parametric study of laser induced ablation-oxidation on porous silicon surfaces," Journal of Physics: Condensed Matter, vol. 20, p. 265009, 2008. 
[35] A. Osipov, P. Patzner, and P. Hess, "Kinetics of laser-induced oxidation of silicon near room temperature," Applied Physics A: Materials Science 83 Processing, vol. 82, no. 2, pp. 275-280, 2006.

[36] J. Plummer, M. Deal, P. Griffin et al., Silicon VLSI technology: fundamentals, practice and modeling. Prentice Hall Upper Saddle River, NJ, 2000, vol. 4.

[37] B. Deal and A. Grove, "General relationship for the thermal oxidation of silicon," Journal of Applied Physics, vol. 36, no. 12, pp. 3770-3778, 1965.

[38] B. Tan, A. Dalili, and K. Venkatakrishnan, "High repetition rate femtosecond laser nano-machining of thin films," Applied Physics A: Materials Science 83 Processing, vol. 95, no. 2, pp. 537-545, 2009.

[39] E. Gamaly, A. Rode, and B. Luther-Davies, "Ultrafast ablation with high-pulse-rate lasers. part i: Theoretical considerations," Journal of applied physics, vol. 85, p. 4213, 1999.

[40] A. Rode, B. Luther-Davies, and E. Gamaly, "Ultrafast ablation with high-pulse-rate lasers. part ii: Experiments on laser deposition of amorphous carbon films," Journal of applied physics, vol. 85, p. 4222, 1999.

[41] A. Kiani, K. Venkatakrishnan, and B. Tan, "Direct laser writing of amorphous silicon on si-substrate induced by high repetition femtosecond pulses," Journal of Applied Physics, vol. 108, p. 074907, 2010.

[42] J. Bronski, M. Segev, and M. Weinstein, "Mathematical frontiers in optical solitons," 
Proceedings of the National Academy of Sciences of the United States of America, vol. 98 , no. 23, p. $12872,2001$.

[43] P. Agostini and L. DiMauro, "The physics of attosecond light pulses," Reports on Progress in Physics, vol. 67, p. 813, 2004.

[44] J. Shah, Ultrafast spectroscopy of semiconductors and semiconductor nanostructures. Springer Verlag, 1999, vol. 115.

[45] A. Zewail, "Femtochemistry," The Journal of Physical Chemistry, vol. 97, no. 48, pp. $12427-12446,1993$.

[46] D. Wiersma, Femtosecond Reaction Dynamics: Proceedings of the Colloquium'Femtosecond Reaction Dynamics', Amsterdam, 17-19 May 1993. North Holland, 1994, vol. 42.

[47] P. Liu, R. Yen, N. Bloembergen, and R. Hodgson, "Picosecond laser-induced melting and resolidification morphology on si," Applied Physics Letters, vol. 34, no. 12, pp. 864-866, 1979.

[48] P. Woodard and J. Dryden, "Thermal analysis of a laser pulse for discrete spot surface transformation hardening," Journal of applied physics, vol. 85, no. 5, pp. 2488-2496, 1999.

[49] A. Kiani, K. Venkatakrishnan, and B. Tan, "Enhancement of the optical absorption of thin-film of amorphorized silicon for photovoltaic energy conversion," Solar Energy, vol. 85, no. 9, pp. 1817-1823, September 2011, also available as: http://dx.doi.org/ 10.1016/j.solener.2011.04.020. 
[50] M. Bertolotti, "Physical processes in laser-materials interactions," Univ. of Rome, Rome, Tech. Rep., 1983.

[51] A. Kiani, K. Venkatakrishnan, and B. Tan, "Direct patterning of silicon oxide on sisubstrate induced by femtosecond laser," Optics Express, vol. 18, no. 3, pp. 1872-1878, 2010.

[52] K. Venkatakrishnan, B. Tan, P. Stanley, L. Lim, and B. Ngoi, "Femtosecond pulsed laser direct writing system," Optical Engineering, vol. 41, p. 1441, 2002.

[53] A. Kiani, K. Venkatakrishnan, and B. Tan, "Maskless lithography using patterned amorphous silicon layer induced by femtosecond laser irradiation," Nanotechnogy, vol. 2, pp. $276-279,2010$.

[54] A. Kiani, K. Venkatakrishnan, B. Tan, and V. Venkataramanan, "Maskless lithography using silicon oxide etch-stop layer induced by megahertz repetition femtosecond laser pulses," Optics Express, vol. 19, no. 11, pp. 10 834-10842, 2011.

[55] N. Rouhi, B. Esfandyarpour, S. Mohajerzadeh, P. Hashemi, B. Hekmat-Shoar, and M. Robertson, "Low temperature high quality growth of silicon-dioxide using oxygenation of hydrogenation-assisted nano-structured silicon thin films," in MRS Proceedings, vol. 989, no. 1. Cambridge Univ Press, 2007.

[56] J. Blanc, "A revised model for the oxidation of si by oxygen," Applied Physics Letters, vol. 33, no. 5, pp. 424-426, 1978.

[57] V. Samalam, "Theoretical model for the oxidation of silicon," Applied physics letters, vol. 47, no. 7, pp. 736-737, 1985. 
[58] A. Fargeix and G. Ghibaudo, "Role of stress on the parabolic kinetic constant for dry silicon oxidation," Journal of applied physics, vol. 56, no. 2, pp. 589-591, 1984.

[59] H. Massoud, J. Plummer, and E. Irene, "Thermal oxidation of silicon in dry oxygen growth-rate enhancement in the thin regime," Journal of the Electrochemical Society, vol. 132, p. 2685, 1985.

[60] A. Ancona, F. Röser, K. Rademaker, J. Limpert, S. Nolte, and A. Tünnermann, "High speed laser drilling of metals using a high repetition rate, high average power ultrafast fiber cpa system," Optics express, vol. 16, no. 12, pp. 8958-8968, 2008.

[61] S. Panchatsharam, B. Tan, and K. Venkatakrishnan, "Femtosecond laser-induced shockwave formation on ablated silicon surface," Journal of Applied Physics, vol. 105, no. 9, pp. 093 103-093 103, 2009.

[62] J. Bonse, K. Brzezinka, and A. Meixner, "Modifying single-crystalline silicon by femtosecond laser pulses: an analysis by micro raman spectroscopy, scanning laser microscopy and atomic force microscopy," Applied surface science, vol. 221, no. 1, pp. 215$230,2004$.

[63] A. Vorobyev and C. Guo, "Direct observation of enhanced residual thermal energy coupling to solids in femtosecond laser ablation," Applied Physics Letters, vol. 86, p. 011916, 2005.

[64] H. Shanks, P. Maycock, P. Sidles, and G. Danielson, "Thermal conductivity of silicon from 300 to 1400 k," Physical Review, vol. 130, no. 5, p. 1743, 1963. 
[65] J. Liu, R. Yen, H. Kurz, and N. Bloembergen, "Phase transformation on and charged particle emission from a silicon crystal surface, induced by picosecond laser pulses," Applied Physics Letters, vol. 39, no. 9, pp. 755-757, 1981.

[66] J. Bonse, S. Baudach, J. Krüger, W. Kautek, and M. Lenzner, "Femtosecond laser ablation of silicon-modification thresholds and morphology," Applied Physics A: Materials Science 83 Processing, vol. 74, no. 1, pp. 19-25, 2002.

[67] C. Bradley, W. Anderson, J. McClelland, and R. Celotta, "Nanofabrication via atom optics," Applied surface science, vol. 141, no. 3-4, pp. 210-218, 1999.

[68] P. Patzner, A. Osipov, and P. Hess, "Photoinduced self-limited low-temperature growth of ultra-thin silicon-oxide films with water vapor," Applied Physics A: Materials Science 83 Processing, vol. 85, no. 2, pp. 145-150, 2006.

[69] B. Stuart, M. Feit, S. Herman, A. Rubenchik, B. Shore, and M. Perry, "Nanosecondto-femtosecond laser-induced breakdown in dielectrics," Physical Review B, vol. 53, no. 4, p. 1749, 1996.

[70] K. Venkatakrishnan, P. Stanley, N. Sivakumar, B. Tan, and L. Lim, "Effect of scanning resolution and fluence fluctuation on femtosecond laser ablation of thin films," Applied Physics A: Materials Science ES Processing, vol. 77, no. 5, pp. 655-658, 2003.

[71] S. Eaton, H. Zhang, M. Ng, J. Li, W. Chen, S. Ho, and P. Herman, "Transition from thermal diffusion to heat accumulation in high repetition rate femtosecond laser writing of buried optical waveguides," Optics express, vol. 16, no. 13, pp. 9443-9458, 2008. 
[72] N. Ma, H. Ma, M. Zhong, J. Yang, Y. Dai, G. Ye, Z. Yue, G. Ma, and J. Qiu, "Direct precipitation of silver nanoparticles induced by a high repetition femtosecond laser," Materials Letters, vol. 63, no. 1, pp. 151-153, 2009.

[73] A. Borowiec and H. Haugen, "Femtosecond laser micromachining of grooves in indium phosphide," Applied Physics A: Materials Science \& Processing, vol. 79, no. 3, pp. $521-529,2004$.

[74] J. Ell, T. Crosby, J. Peterson, K. Carter, and J. Watkins, "Formation of sio2 airgap patterns through scco2 infusion of nil patterned phema," Chemistry of Materials, vol. 22 , no. 4, pp. 1445-1451, 2010.

[75] G. Della Giustina, M. Guglielmi, G. Brusatin, M. Prasciolu, and F. Romanato, "Electron beam writing of epoxy based sol-gel materials," Journal of Sol-Gel Science and Technology, vol. 48, no. 1, pp. 212-216, 2008.

[76] M. Flores-Arias, A. Castelo, C. Gómez-Reino, and G. de La Fuente, "Phase diffractive optical gratings on glass substrates by laser ablation," Optics Communications, vol. 282, no. 6, pp. 1175-1178, 2009.

[77] K. Aissou, M. Kogelschatz, T. Baron, and P. Gentile, "Self-assembled block polymer templates as high resolution lithographic masks," Surface science, vol. 601, no. 13, pp. 2611-2614, 2007.

[78] R. Pai, R. Humayun, M. Schulberg, A. Sengupta, J. Sun, and J. Watkins, "Mesoporous silicates prepared using preorganized templates in supercritical fluids," Science, vol. 303, no. 5657, pp. 507-510, 2004. 
[79] D. Yin, S. Horiuchi, and T. Masuoka, "Lateral assembly of metal nanoparticles directed by nanodomain control in block copolymer thin films," Chemistry of materials, vol. 17, no. 3, pp. 463-469, 2005.

[80] E. Carvalho, M. Alves, E. Braga, and L. Cescato, "Sio $<\operatorname{sub}>2</$ sub $>$ single layer for reduction of the standing wave effects in the interference lithography of deep photoresist structures on si," Microelectronics journal, vol. 37, no. 11, pp. 1265-1270, 2006.

[81] C. Guo, S. Cao, P. Jiang, Y. Fang, J. Zhang, Y. Fan, Y. Wang, W. Xu, Z. Zhao, and Q. Liu, "Grayscale photomask fabricated by laser direct writing in metallic nanofilms," Opt. Express, vol. 17, no. 22, pp. 19 981-19 987, 2009.

[82] K. Venkatakrishnan, B. Ngoi, P. Stanley, L. Lim, B. Tan, and N. Sivakumar, "Laser writing techniques for photomask fabrication using a femtosecond laser," Applied Physics A: Materials Science \& Processing, vol. 74, no. 4, pp. 493-496, 2002.

[83] J. Hastings, M. Lim, J. Goodberlet, and H. Smith, "Optical waveguides with apodized sidewall gratings via spatial-phase-locked electron-beam lithography," Journal of Vacuum Science 83 Technology B: Microelectronics and Nanometer Structures, vol. 20, p. $2753,2002$.

[84] J. Hastings, F. Zhang, and H. Smith, "Nanometer-level stitching in raster-scanning electron-beam lithography using spatial-phase locking," Journal of Vacuum Science 83 Technology B: Microelectronics and Nanometer Structures, vol. 21, p. 2650, 2003.

[85] G. Atkinson, F. Stratton, R. Kubena, and J. Wolfe, "30 nm resolution zero proxim- 
ity lithography on high-z substrates," Journal of Vacuum Science 83 Technology B: Microelectronics and Nanometer Structures, vol. 10, no. 6, pp. 3104-3108, 1992.

[86] J. Spallas, C. Silver, and L. Muray, "Arrayed miniature electron beam columns for mask making," Journal of Vacuum Science 83 Technology B: Microelectronics and Nanometer Structures, vol. 24, p. 2892, 2006.

[87] M. Walsh and H. Smith, "Method for reducing hyperbolic phase in interference lithography," Journal of Vacuum Science \&3 Technology B: Microelectronics and Nanometer Structures, vol. 19, p. 2347, 2001.

[88] P. Konkola, C. Chen, R. Heilmann, C. Joo, J. Montoya, C. Chang, and M. Schattenburg, "Nanometer-level repeatable metrology using the nanoruler," Journal of Vacuum Science ES Technology B: Microelectronics and Nanometer Structures, vol. 21, p. 3097, 2003.

[89] T. Sandstrom, A. Bleeker, J. Hintersteiner, K. Troost, J. Freyer, and K. van der Mast, "Oml: optical maskless lithography for economic design prototyping and smallvolume production [5377-69]," in PROCEEDINGS-SPIE THE INTERNATIONAL SOCIETY FOR OPTICAL ENGINEERING, no. 2. International Society for Optical Engineering; 1999, 2004, pp. 777-787.

[90] I. Moran, A. Briseno, S. Loser, and K. Carter, "Device fabrication by easy soft imprint nano-lithography," Chemistry of Materials, vol. 20, no. 14, pp. 4595-4601, 2008.

[91] S. Krämer, R. Fuierer, and C. Gorman, "Scanning probe lithography using selfassembled monolayers," detail, vol. 41, p. 44, 2003. 
[92] J. Wei and D. Ginger, "A direct-write single-step positive etch resist for dip-pen nanolithography," Small, vol. 3, no. 12, pp. 2034-2037, 2007.

[93] K. Venkatakrishnan and B. Tan, "Interconnect microvia drilling with a radially polarized laser beam," Journal of Micromechanics and Microengineering, vol. 16, p. 2603, 2006.

[94] N. Kawasegi, N. Morita, S. Yamada, N. Takano, T. Oyama, S. Momota, J. Taniguchi, and I. Miyamoto, "Depth control of a silicon structure fabricated by $100<\mathrm{i}>\mathrm{q}</ \mathrm{i}>$ kev ar ion beam lithography," Applied surface science, vol. 253, no. 6, pp. 3284-3291, 2007.

[95] M. Archer and F. Ligler, "Fabrication and characterization of silicon micro-funnels and tapered micro-channels for stochastic sensing applications," Sensors, vol. 8, no. 6, pp. 3848-3872, 2008.

[96] H. Sai, Y. Kanamori, K. Arafune, Y. Ohshita, and M. Yamaguchi, "Light trapping effect of submicron surface textures in crystalline si solar cells," Progress in Photovoltaics: Research and Applications, vol. 15, no. 5, pp. 415-423, 2007.

[97] Y. Song, S. Bae, J. Yu, and Y. Lee, "Closely packed and aspect-ratio-controlled antireflection subwavelength gratings on gaas using a lenslike shape transfer," Optics letters, vol. 34, no. 11, pp. 1702-1704, 2009.

[98] M. Green, K. Emery, Y. Hishikawa, and W. Warta, "Solar cell efficiency tables (version 31)," Progress in Photovoltaics, vol. 16, no. 1, p. 61, 2008. 
[99] Y. Kameya and K. Hanamura, "Enhancement of solar radiation absorption using nanoparticle suspension," Solar Energy, vol. 85, no. 2, pp. 299-307, 2011.

[100] K. Yamamoto, A. Nakajima, M. Yoshimi, T. Sawada, S. Fukuda, T. Suezaki, M. Ichikawa, Y. Koi, M. Goto, T. Meguro et al., "A high efficiency thin film silicon solar cell and module," Solar Energy, vol. 77, no. 6, pp. 939-949, 2004.

[101] J. Arch, J. Werner, and E. Bauser, "Hall effect analysis of liquid phase epitaxy silicon for thin film solar cells," Solar energy materials and solar cells, vol. 29, no. 4, pp. 387-396, 1993.

[102] Z. Shi, W. Zhang, G. Zheng, V. Chin, A. Stephens, M. Green, and R. Bergmann, "The effects of solvent and dopant impurities on the performance of lpe silicon solar cells," Solar energy materials and solar cells, vol. 41, pp. 53-60, 1996.

[103] Y. Akimov, K. Ostrikov, and E. Li, "Surface plasmon enhancement of optical absorption in thin-film silicon solar cells," Plasmonics, vol. 4, no. 2, pp. 107-113, 2009.

[104] Y. Lu and A. Lal, "High-efficiency ordered silicon nano-conical-frustum array solar cells by self-powered parallel electron lithography," Nano letters, vol. 10, no. 11, pp. 4651-4656, 2010.

[105] L. Tsakalakos, J. Balch, J. Fronheiser, B. Korevaar, O. Sulima, and J. Rand, "Silicon nanowire solar cells," Applied Physics Letters, vol. 91, no. 23, pp. 233117-233117, 2007.

[106] D. Ge, V. Domnich, and Y. Gogotsi, "High-resolution transmission electron micro- 
scopy study of metastable silicon phases produced by nanoindentation," Journal of applied physics, vol. 93, p. 2418, 2003.

[107] N. Park, T. Kim, and S. Park, "Band gap engineering of amorphous silicon quantum dots for light-emitting diodes," Applied Physics Letters, vol. 78, p. 2575, 2001. 